\title{
Statistical Study of the Swift X-ray Flash and X-ray Rich Gamma-Ray Bursts
}

\author{
Xiongwei $\mathrm{Bi}^{1,2}$, Jirong $\mathrm{Mao}^{3,4,2}$, Chuanxi $\mathrm{Liu}^{3,4,5}$, and Jin-Ming Bai ${ }^{3,4,2}$ \\ jirongmao@mail.ynao.ac.cn
}

\begin{abstract}
We build a comprehensive sample to statistically describe the properties of X-ray flashes (XRFs) and X-ray riches (XRRs) from the latest third Swift Burst Alert Telescope (BAT3) catalog of Gamma-ray bursts (GRBs). We obtain 81 XRFs, 540 XRRs, and 394 classical GRBs (C-GRBs). We statistically explore the different properties of the $\gamma$-ray prompt emission, the X-ray emission, the Xray lightcurve type, the association with supernovae (SNe), and the host galaxy properties for these sources. We confirm that most XRFs/XRRs are long GRBs with low values of peak energy $E_{\text {peak }}^{o b s}$ and they are low-luminosity GRBs. XRFs, XRRs, and C-GRBs follow the same $E_{X, i s o}-E_{\gamma, i s o}-E_{p e a k, z}$ correlations. Compared to the classical GRBs, XRFs are favorable to have the association with SN explosions. We do not find any significant differences of redshift distribution and host galaxy properties among XRFs, XRRs, and C-GRBs. We also discuss some observational biases and selection effects that may affect on our statistical results. The GRB detectors with wide energy range and low energy threshold are expected for the XRF/XRR research in the future.
\end{abstract}

Subject headings: gamma rays: general — radiation mechanisms: non-thermal

\footnotetext{
${ }^{1}$ Department of Physics, Honghe University, 661199 Mengzi, China

${ }^{2}$ Key Laboratory for the Structure and Evolution of Celestial Objects, Chinese Academy of Sciences, 650011 Kunming, China

${ }^{3}$ Yunnan Observatories, Chinese Academy of Sciences, 650011 Kunming, Yunnan Province, China

${ }^{4}$ Center for Astronomical Mega-Science, Chinese Academy of Sciences, 20A Datun Road, Chaoyang District, 100012 Beijing, China

${ }^{5}$ University of Chinese Academy of Sciences, 100049 Beijing, China
} 


\section{Introduction}

In addition to the classical long/short dichotomy, gamma-ray bursts (GRBs) have two special subclasses: X-ray flashes (XRFs) and X-ray riches (XRRs). XRFs are the GRBs characterized by the faint signals in the gamma-ray energy band. XRRs, which belong to an intermediate class between XRFs and classical GRBs (C-GRBs), have stronger X-ray emission compared to their gamma-ray emission (e.g, Barraud et al. 2003; Kippen et al. 2003; Amati et al. 2004; Sakamoto et al. 2005, 2008; D'Alessio et al. 2006). The physical origin of XRFs and XRRs are still under debate.

$\mathrm{XRF} 050406$ was proposed as a GRB with prelonged central engine activity (Romano et al. 2006). This long-term activity was also observed in XRF 011030 (Galli \& Piro 2006). GRB jet structure affects on the observed GRB energy release, and the off-axis effect may induce the observed XRFs/XRRs (e.g., Yamazaki et al. 2002; Barraud et al. 2005; Granot et al. 2005; Lamb et al. 2005; Xu et al. 2005; Donaghy 2006; Salafia et al. 2016). Observations have provided some further evidence for the off-axis jet injection (e.g., Bulter et al. 2005; Schady et al. 2006; de Ugarte Postigo 2007; Guidorzi et al. 2009). A dynamic transition with a different GRB jet opening angle may also be important to link C-GRBs and XRFs (Mizuta et al. 2006). Alternatively, thermal emission has been thought to be a possible component in the strong X-ray emission of GRBs. Ramirez-Ruiz (2005) proposed a photospheric model that can be used to interpret the dominated X-ray emission of XRFs. Pe'er et al. (2006) calculated the details of the photospheric component of the XRF prompt emission spectrum. XRFs can also be the indicators of the orphan GRB afterglows (Urata et al. 2015). These clues naturally lead to one suggestion that XRFs are low-luminosity GRBs (e.g., Virgili et al. 2009). Moreover, it has been found that supernova (SN) explosions can be associated with XRFs (e.g., XRF 020903, Bersier et al. 2006).

XRF 050215B was the first XRF observed by Swift (Levan et al. 2006), and Swift observational statistics can be well applied to study the physical origins of XRFs and XRRs (Gendre et al. 2007). Sakamoto et al. (2008) built one dataset provided by the Swift Burst Alert telescope (BAT) observation from 2004 December to 2006 September. From that sample, they obtained $10 \mathrm{XRFs}$ and $97 \mathrm{XRRs}$ among a total of 158 GRBs. They studied the prompt emission properties and the X-ray afterglow emission characteristics for XRFs, XRRs, and C-GRBs. Some distinct differences between XRFs and C-GRBs in the prompt emission and the X-ray afterglow emission have been illustrated. This exploration encourages us to comprehensively investigate the physical properties of XRFs/XRRs, the relation between XRFs/XRRs and C-GRBs, and the GRB central engine from the statistical point of view. Thus, a large GRB sample is necessary.

We utilize the latest third Swift-BAT3 catalog (Lien et al. 2016), which contains 1104 
GRBs detected from 2004 December 17 to 2016 December 2, to systematically investigate the statistical properties of XRFs and XRRs. For each GRB, the catalog provides trigger time, coordinates, redshift, GRB duration time $T_{90}$, spectral models for spectral fitting, spectral photon index, observed peak energy $E_{\text {peak }}$, and fluence in difference energy bands. From this catalog, we classify the possible XRFs/XRRs and build a sample to comprehensively analyze the differences of XRFs/XRRs and C-GRBs. We identify 81 XRFs, 540 XRRs, and 394 CGRBs included in the sample and statistically analyze their observational characteristics. We examine the possible associations between XRFs/XRRs and SNe. The host galaxy properties of XRFs are also presented. Some observational biases and selection effects are mentioned.

This paper is organized as follows. We classify XRFs, XRRs, and C-GRBs in Section 2. In Section 3, we present the different properties of XRFs, XRRs, and C-GRBs, respectively. A discussion is provided in Section 4, and conclusions are listed in Section 5. We adopt the cosmological parameters as $H_{0}=70 \mathrm{~km} \mathrm{~s}^{-1} \mathrm{Mpc}^{-1}, \Omega_{\Lambda}=0.7$, and $\Omega_{M}=0.3$. The quoted errors are at the $90 \%$ confidence level unless stated otherwise.

\section{Sample Selection}

We classify XRFs, XRRs, and C-GRBs using the criteria provided by Sakamoto et al. (2008). The definitions of XRF, XRR, and C-GRB based on the fluence ratio of $S(25-$ $50 \mathrm{keV})$ and $S(50-100 \mathrm{keV})$ are:

$$
\begin{array}{r}
S(25-50 \mathrm{keV}) / \mathrm{S}(50-100 \mathrm{keV}) \leq 0.72 \quad(\mathrm{C}-\mathrm{GRB}) \\
0.72<S(25-50 \mathrm{keV}) / \mathrm{S}(50-100 \mathrm{keV}) \leq 1.32 \quad(\mathrm{XRR}) \\
S(25-50 \mathrm{keV}) / \mathrm{S}(50-100 \mathrm{keV})>1.32 \quad(\mathrm{XRF}) .
\end{array}
$$

We obtain 81 XRFs, 540 XRRs, and 394 C-GRBs in the Swift-BAT3 catalog by the selection condition of Equation (1). We summarize the selection results in Table 1. We further consider three additional conditions. First, in order to compare the prompt emission and the X-ray afterglow emission of each XRF/XRR, we exclude 89 sources that have no Swift X-ray Telescope (XRT) data. Thus, we do not clarify these sources as XRFs, XRRs, or C-GRBs. We also specify that 13 sources in our sample have no GRB duration $T_{90}$ numbers. Thus, we cannot clarify them as long-duration GRBs (L-GRBs, defined by $T_{90} \geq 2 \mathrm{~s}$ ) or short-duration GRBs (S-GRBs, defined by $T_{90} \leq 2 \mathrm{~s}$ ). Second, we also select GRBs that have the photon index $\alpha_{P L}<-2.0$ with a power-low fitting in the BAT3 dataset and identify them as XRFs. Third, we note the sources having values of $E_{\text {peak }}^{o b s}$, which can be found in

the BAT3 dataset. We also check whether these selected sources have $E_{\text {peak }}^{o b s}$ values in other datasets. Finally, we list XRFs and XRRs in Table 2. 
The distributions with the fluence ratio of $S(25-50 \mathrm{keV}) / S(50-100 \mathrm{keV})$ for the total 1015 GRBs in the BAT3 catalog are shown in Figure 1. XRFs, XRRs, and C-GRBs in the sample have the fractions of $(8.0 \pm 0.9) \%,(53.2 \pm 2.2) \%$, and $(38.8 \pm 1.9) \%$, respectively. The smaller sample given by Sakamoto et al. (2008) contains 158 GRBs. There are 10 XRFs, 97 XRRs, and $51 \mathrm{C}-G R B s$. XRFs, XRRs, and C-GRBs in their sample have the fractions of $(6.3 \pm 2.0) \%,(61.4 \pm 6.2) \%$, and $(32.3 \pm 4.5) \%$, respectively. It seems that our classified results are roughly consistent with those of Sakamoto et al. (2008). Here, we pay attention to four special cases: (1) GRB 050219B was identified as XRR by Sakamoto et al. (2008), while it is classified as C-GRB in our work. (2) GRB 050815 was identified as XRR by Sakamoto et al. (2008), while it is classified as XRF in our work. (3) There are 10 GRBs (GRB 050824, GRB 060512, GRB 060923B, GRB 060926, GRB070714A, GRB070721A, GRB080218B, GRB080515, GRB080520, and GRB081007) that have no fluences of $S(25-$ $50 \mathrm{keV})$ and/or $S(50-100 \mathrm{keV})$ in BAT3 catalog, and we find the fluences of $S(25-50 \mathrm{keV})$ and/or $S$ (50-100 keV) from the Swift-BAT2 catalog (Sakamoto et al. 2011). (4) There are 11 XRFs (XRF 050406, XRF 050416A, XRF 050819, XRF 060428B, XRF 060805A, XRF 061218, XRF 070126, XRF 080218B, XRF 080315, XRF 080822B, and XRF 160525A) show a fluence ratio of $S(25-50 \mathrm{keV}) / S(50-100 \mathrm{keV})$ larger than 3.01.

We plot the fluence ratio $S(25-50 \mathrm{keV}) / S(50-100 \mathrm{keV})$ versus the BAT-observed GRB duration $T_{90}$ in Figure 2. We calculate the fractions of L-GRBs and S-GRBs for XRFs, XRRs, and C-GRBs in our sample, respectively. Our findings are as follows: (1) For XRFs, there are 70 L-GRBs, 3 S-GRBs, and 8 duration-unclear sources, and the fractions are $(86.4 \pm 10.3) \%,(3.7 \pm 2.1) \%$, and $(9.9 \pm 3.5) \%$, respectively. (2) For XRRs, there are 509 L-GRBs, 27 S-GRBs, and 4 duration-unclear sources, and the fractions are $(94.3 \pm 4.2) \%$, $(5.0 \pm 1.0) \%$, and $(0.7 \pm 0.4) \%$, respectively. (3) For C-GRBs, there are $328 \mathrm{~L}-\mathrm{GRBs}, 65$ $\mathrm{S}-\mathrm{GRBs}$, and 1 duration-unclear source, and the fractions are $(83.2 \pm 4.6) \%,(16.5 \pm 2.0) \%$, and $(0.3 \pm 0.3) \%$, respectively. We note that XRFs and XRRs have less S-GRB proportion compared with $\mathrm{C}-\mathrm{GRB} \mathrm{s}^{2}$. In the meantime, we also report the fraction of XRFs, XRRs, and C-GRBs for L-GRB and S-GRB classes. Three S-GRBs as XRFs have the fraction of $(3.2 \pm 1.8) \%$. Twenty-seven S-GRBs as XRRs have the fraction of (28.4 \pm 5.5$) \%$. Sixty-five S-GRBs as C-GRBs have the fraction of $(68.4 \pm 8.5) \%$. Seventy L-GRBs as XRFs have

\footnotetext{
${ }^{1} \mathrm{XRF} 080315$ has the largest fluence ratio of $53.8 \pm 143.0$ but with large error. This source has no X-ray afterglow from Swift-XRT detection. Due to the lack of X-ray nondetection and the marginal BAT detection (Page \& Gehrels 2008), we include this source in Table 1, 2 and 3, but we exclude it in all Figures of this paper. We also do not find any other notable issues for this burst.

${ }^{2}$ There are only three XRFs (XRF 090417A, XRF 110112A, and XRF 140622A) that are S-GRBs. GRB 110112A has no host galaxy evidence (Fong et al. 2013; Tunnicliffe et al. 2014). We do not find any other notable information for the short-duration XRFs.
} 
the fraction of $(7.7 \pm 0.9) \%$. Five hundred and nine L-GRBs as XRRs have the fraction of $(56.1 \pm 2.5) \%$. Three hundred and twenty-eight L-GRBs as C-GRBs have the fraction of $(36.2 \pm 2.0) \%$.

\section{Statistical Analysis}

\subsection{The Prompt Emission Properties}

We collect $E_{\text {peak }}^{\text {obs }}$ values of GRBs from the literature (e.g., Amati et al. 2008, 2009; Sakamoto et al. 2008, 2011; Grupe et al. 2013; D'Avanzo et al. 2014; Liang et al. 2015; Lien et al. 2016; Zaninoni et al. 2016), and we obtain the $E_{\text {peak }}^{\text {obs }}$ values for $77 \mathrm{XRFs}, 460 \mathrm{XRRs}$, and 265 C-GRBs. The fluence ratio $S(25-50 \mathrm{keV}) / S(50-100 \mathrm{keV})$ versus $E_{\text {peak }}^{\text {obs }}$ is shown in Figure 3. We clearly see the different occupied regions of XRFs, XRRs, and C-GRBs in the Figure. It was shown in the fluence ratio- $E_{\text {peak }}^{o b s}$ plot provided by Sakamoto et al. (2008) a gap of $S(25-50 \mathrm{keV}) / S(50-100 \mathrm{keV})$ fluence ratio from 0.8 to 1.2 , and Sakamoto et al. (2008) suggested that this gap is the result of selection effects. However, we do not find this gap in Figure 3, because we take a large sample from the BAT3 catalog. We further show the different $E_{\text {peak }}^{o b s}$ distributions for XRFs, XRRs, and C-GRBs in Figure 4. In order to quantitatively distinguish the different $E_{\text {peak }}^{o b s}$ properties to XRFs, XRRs, and C-GRBs, we use a nonparametric two-sample Kolmogorov-Smirnov (K-S) test to examine the different $E_{\text {peak }}^{o b s}$ distributions for the XRF/XRR samples, the XRR/C-GRB samples, and the XRF/C-GRB samples, respectively. Because the K-S probability numbers are very small (the $P$-values are far less than 0.0001), we confirm that the $E_{\text {peak }}^{o b s}$ distributions among XRFs, XRRs, and C-GRBs have significant differences.

The $E_{\text {peak }}^{\text {obs }}$ distribution of XRFs ranges from $0.9 \mathrm{keV}$ to $80.0 \mathrm{keV}$, with a mean value of $24.3 \pm 1.6 \mathrm{keV}$. The $E_{\text {peak }}^{\text {obs }}$ distribution of XRRs ranges from $1.2 \mathrm{keV}$ to $1780.0 \mathrm{keV}$, with a mean value of $105.6 \pm 5.3 \mathrm{keV}$. The $E_{\text {peak }}^{\text {obs }}$ distribution of C-GRBs ranges from $64.6 \mathrm{keV}$ to $2602.8 \mathrm{keV}$, with a mean value of $257.7 \pm 14.1 \mathrm{keV}$. It is clear that XRFs and XRRs have smaller $E_{\text {peak }}^{\text {obs }}$ values compared with C-GRBs. Our results are consistent with those of Sakamoto et al. (2005, 2008). We confirm that XRFs and XRRs release their prompt energies mostly in the X-ray band.

We also investigate the correlation between peak energy $E_{\text {peak }}^{\text {obs }}$ and the fluence $S(15-150$ $\mathrm{keV}$ ) for all GRBs in our sample. The data are plotted in Figure 5. In principle, the effect of the data errors should be taken into account when we perform the correlation fitting. In this paper, we adopt the maximum likelihood method that has been well applied for the $E_{\text {peak,z }}-E_{\gamma \text {,iso }}$ correlation fitting given by Amati et al. (2008). We use the maximum 
likelihood method and obtain the correlation fitting as $\log \left(E_{\text {peak }}^{\text {obs }}\right)(\mathrm{keV})=(2.96 \pm 0.13)+$ $(0.16 \pm 0.02) \log [S(15-150)] \mathrm{keV}$ with the extrinsic scatter $\sigma=0.39 \pm 0.01$. We also plot the correlation of $\log \left(E_{\text {peak }}^{\text {obs }}\right)(\mathrm{keV})=(5.46 \pm 0.25)+(0.62 \pm 0.14) \log [S(15-150 \mathrm{keV})]$ that was given by Sakamoto et al. (2008). We see that our fitting result is different from that of Sakamoto et al. (2008). In order to clarify the difference of this correlation among XRFs, XRRs, and C-GRBs, we separate C-GRBs as one group and put XRRs and XRFs as the other group. We obtain the fitting for C-GRBs as $\log \left(E_{\text {peak }}^{o b s}\right)(\mathrm{keV})=(3.01 \pm 0.13)+(0.12 \pm 0.02) \log [S(15-$ $150)] \mathrm{keV}$ with the extrinsic scatter $\sigma=0.26 \pm 0.01$, and the fitting for XRRS and C-GRBs as $\log \left(E_{\text {peak }}^{\text {obs }}\right)(\mathrm{keV})=(2.67 \pm 0.16)+(0.13 \pm 0.03) \log [S(15-150)] \mathrm{keV}$ with the extrinsic scatter $\sigma=0.36 \pm 0.01$. Therefore, although the difference between XRFs and XRRs/C-GRBs is clear, it seems no significant difference between XRFs/XRRs and C-GRBs because XRRs and C-GRBs has large overlap region seen in Fig. 5.

\subsection{The Observed Properties with Redshift}

The redshift distributions of the XRFs, XRRs, and C-GRBs are shown in Figure 6 . Using the K-S test to the redshift distributions for the XRFs and XRRs samples, the XRRs and C-GRBs samples, and the XRFs and C-GRBs samples, we find that K-S test probabilities are $P=0.13, P=0.36$, and $P=0.13$, respectively. The $\mathrm{K}-\mathrm{S}$ test results confirm that there are not significant differences among XRFs, XRRs, and C-GRBs for the redshift distribution.

We also plot the BAT-observed duration $T_{90}$ and the fluence $S(15-150 \mathrm{keV})$ as a function of redshift in Figure 7 and Figure 8, respectively. We do not find significant differences of $T_{90}$ and $S(15-150 \mathrm{keV})$ distributions among XRFs, XRRs, and C-GRBs, and we do not see significant redshift evolutions of $T_{90}$ and $S(15-150 \mathrm{keV})$ for XRFs, XRRs, and C-GRBs.

\subsection{The Correlations among $E_{X, i s o}, E_{\gamma, i s o}$, and $E_{p e a k, z}$}

There is a universal correlation among the isotropic prompt energy $E_{\gamma, \text { iso }}$ emitted in the rest frame $1-10^{4} \mathrm{keV}$ energy band, the rest frame energy peak of the prompt emission energy spectrum $E_{\text {peak,z }}$ in which $E_{\text {peak,z }}=(1+z) E_{\text {peak }}^{\text {obs }}$, and the X-ray energy emitted in the rest frame $0.3-10 \mathrm{keV}$ energy band $E_{X, \text { iso }}$ for GRBs (e.g., Bernardini et al. 2012; Margutti et al. 2013; D'Avanzo et al. 2014; Zaninoni et al. 2016). In order to check whether XRFs, XRRs, and C-GRBs in our sample follow this correlation, respectively, we collect the $E_{\gamma, i s o}$ and $E_{X, i s o}$ data of the GRBs in our sample from Amati et al. (2008), Margutti et al. (2013), and Liang et al. (2015). First, the relation between $E_{X, i s o}$ and 
$E_{\gamma, i s o}$ is shown in Figure 9. We perform the maximum likelihood method and obtain the fitting of $\left(E_{X, i s o}\right)=(13.31 \pm 2.73)+(0.73 \pm 0.05) \log \left(E_{\gamma, i s o}\right)$ with the extrinsic scatter of $\sigma=0.57 \pm 0.04$. The $E_{X, i s o}-E_{\gamma, i s o}$ relation is consistent with that derived by Margutti et al. (2013). Second, we also investigate the correlation between $E_{p e a k, z}$ and $E_{\gamma, i s o}$. The result is shown in Figure 10. The correlation fitted by the maximum likelihood method is $\log \left(E_{\text {peak }, z}\right)(\mathrm{keV})=(2.17 \pm 0.04)+(0.46 \pm 0.03) \log \left[E_{\gamma, \text { iso }} /\left(10^{52} \mathrm{erg}\right)\right]$ with the extrinsic scatter of $\sigma=0.26 \pm 0.02$. Our result is consistent with the correlation reported by Amati (2006). Third, in Figure 11, we present the relation between $E_{X, i s o}$ and $E_{p e a k, z}$ by the maximum likelihood method with the fitting of $\log \left(E_{X, i s o}\right)=(49.69 \pm 0.27)+(0.75 \pm 0.11) \log \left(E_{\text {peak }, z}\right)$, and the extrinsic scatter is $\sigma=0.73 \pm 0.04$. Our result is consistent with that of Margutti et al. (2013). Finally, the $E_{X, i s o}-E_{\gamma, i s o}-E_{p e a k, z}$ relation is shown in Figure 12. The result with the fitting of $\log \left(E_{X, i s o}\right)=(4.78 \pm 2.79)+(0.92 \pm 0.06)\left(\log E_{\gamma, \text { iso }}-0.60 \log \left(E_{\text {peak }}\right)\right.$ and the extrinsic scatter of $\sigma=0.44 \pm 0.04$ produced by the maximum likelihood method is consistent with that of Margutti et al. (2013) as well.

We also examine the difference between XRFs/XRRs and C-GRBs from the above correlations. We separate XRFs/XRRs and C-GRBs as two groups. From Figure 9, we obtain $\left(E_{X, i s o}\right)=(9.85 \pm 2.77)+(0.79 \pm 0.05) \log \left(E_{\gamma, \text { iso }}\right)$ with the extrinsic scatter of $\sigma=0.68 \pm$ 0.07 for C-GRBs and $\left(E_{X, i s o}\right)=(13.45 \pm 2.53)+(0.73 \pm 0.05) \log \left(E_{\gamma, i s o}\right)$ with the extrinsic scatter of $\sigma=0.39 \pm 0.04$ for XRRs/XRFs. Thus, we do not find significant difference in this correlation. we analyze the data in Figure 10 that the relation of $\log \left(E_{\text {peak }, z}\right)(\mathrm{keV})=(2.83 \pm$ $0.06)+(0.11 \pm 0.05) \log \left[E_{\gamma, \text { iso }} /\left(10^{52} \mathrm{erg}\right)\right]$ with the extrinsic scatter of $\sigma=0.31 \pm 0.04$ is for CGRBs and the relation of $\log \left(E_{\text {peak }, z}\right)(\mathrm{keV})=(2.11 \pm 0.03)+(0.41 \pm 0.04) \log \left[E_{\gamma, \text { iso }} /\left(10^{52} \mathrm{erg}\right)\right]$ with the extrinsic scatter of $\sigma=0.23 \pm 0.03$ is for XRRs/XRFs. Thus, we clearly see the difference between XRRs/XRFs and C-GRBs in this correlation. From Figure 11, We obtain the relation of $\log \left(E_{X, i s o}\right)=(48.07 \pm 1.03)+(1.24 \pm 0.35) \log \left(E_{\text {peak }, z}\right)$ with the extrinsic scatter of $\sigma=0.86 \pm 0.09$ for C-GRBs and the relation of $\log \left(E_{X, i s o}\right)=(49.46 \pm 0.28)+$ $(0.90 \pm 0.12) \log \left(E_{\text {peak }, z}\right)$ with the extrinsic scatter of $\sigma=0.62 \pm 0.05$ for XRRs/XRFs. Thus, it seems that XRRs/XRFs and C-GRBs have no significant difference in this correlation. Finally, from Figure 12, we obtain the relation of $\log \left(E_{X, i s o}\right)=(5.67 \pm 3.36)+(0.90 \pm$ $0.07)\left(\log E_{\gamma, \text { iso }}-0.6 \log \left(E_{\text {peak }}\right)\right.$ with the extrinsic scatter of $\sigma=0.54 \pm 0.08$ for C-GRBs and the relation of $\log \left(E_{X, i s o}\right)=(4.10 \pm 2.88)+(0.93 \pm 0.06)\left(\log E_{\gamma, \text { iso }}-0.60 \log \left(E_{\text {peak }}\right)\right.$ with the extrinsic scatter of $\sigma=0.36 \pm 0.05$. Therefore, we do not find any significant difference between XRRs/XRFs and C-GRBs in this correlation.

In order to investigate the $\gamma$-ray isotropic-equivalent luminosity $\left(L_{\gamma, i s o}\right)$ distributions for XRFs, XRRs, and C-GRBs, we collect the $L_{\gamma, \text { iso }}$ values of the GRBs in our sample from D'Avanzo et al. (2014), Liang et al. (2015), and Cano et al. (2017). We obtained 55 sources with $L_{\gamma, \text { iso }}$ values, including $5 \mathrm{XRFs}, 26 \mathrm{XRRs}$, and $24 \mathrm{C}$-GRBs. The $L_{\gamma, \text { iso }}$ 
distributions are shown in Figure 13. Our results are as follows: the $L_{\gamma, \text { iso }}$ values have the range from $2.60 \times 10^{46}$ to $6.89 \times 10^{50} \mathrm{erg} \mathrm{s}^{-1}$ for XRFs; the $L_{\gamma, \text { iso }}$ values are from $1.03 \times 10^{49}$ to $3.51 \times 10^{52} \mathrm{erg} \mathrm{s}^{-1}$ for XRRs; and the $L_{\gamma, \text { iso }}$ values are from $1.20 \times 10^{50}$ to $1.78 \times 10^{53} \mathrm{erg} \mathrm{s}^{-1}$ for C-GRBs. We find that XRFs and XRRs have lower $L_{\gamma, \text { iso }}$ values than C-GRBs. This indicates that XRF sources are low-luminosity GRBs.

\subsection{The X-Ray Lightcurve Shapes}

In order to investigate the X-ray afterglow properties of XRFs, XRRs, and C-GRBs, we simply examine the types of X-ray afterglow light curves for XRFs, XRRs, and C-GRBs from Swift-XRT GRB lightcurve repository ${ }^{3}$. The type definitions of X-ray afterglow light curve given by Margutti et al. (2013) are as follows: type 0 (simple power law), type I (broken power law), type II (broken power law plus power-law decay), and Type III (double broken power laws). We further check the X-ray lightcurve types of XRFs, XRRs, and C-GRBs in our sample. Our results are as follows: (1) There are 33 XRFs having the XRT light curves. For these XRFs, the proportions of Type 0, Type I, Type II, and Type III are $(14.7 \pm 6.6) \%$, $(44.1 \pm 11.4) \%,(32.4 \pm 9.8) \%$, and $(8.8 \pm 5.1) \%$, respectively. (2) There are 198 XRRs having the XRT light curves. For these XRRs, the proportions of Type 0, Type I, Type II, and Type III are $(17.4 \pm 2.9) \%,(30.8 \pm 3.9) \%,(45.3 \pm 4.7) \%$, and $(6.5 \pm 1.8) \%$, respectively. (3) There are $135 \mathrm{C}$-GRBs having the XRT light curves. For these C-GRBs, the proportions of Type 0, Type I, Type II, and Type III are $(25.2 \pm 4.3) \%,(44.4 \pm 5.7) \%,(27.4 \pm 4.5) \%$, and $(3.0 \pm 1.5) \%$, respectively. The statistical results are summarized in Table 3. According to these results, we find that there are not significant differences of the XRT lightcurve type among XRFs, XRRs, and C-GRBs.

\subsection{Investigation of the Association of XRF/XRR with Supernova}

If we propose that XRFs and XRRs are low-luminosity GRBs, it is reasonable to consider the possible association between XRFs/XRRs and SNe (Soderberg et al. 2005; Woosley \& Bloom 2006) 4 . An example is XRF 060218 that is associated with SN 2006aj (Pian et al. 2006). We take the statistical results from Hjorth \& Bloom (2012), and Cano (2013), and

\footnotetext{
${ }^{3}$ http://www.swift.ac.uk/xrt_curves/

${ }^{4}$ Although we focus on low-luminosity GRBs and XRFs in this paper, we note that high-luminosity GRBs may also have SN association. For example, bright GRB 130427A is associated with SN 2013cq (Maselli et al. 2014; Melandri et al. 2014; Vestrand et al. 2014).
} 
Cano et al. (2017). Twenty-three GRBs in our sample are associated with the SN explosion. These GRBs include 6 XRFs (XRF 050416A, XRF 050824, XRF 060218/SN 2006aj, XRF 070419A, XRF 081007/SN 2008hw, and XRF 100316D/SN 2010bh), 14 XRRs (XRR 050525A/SN 2005nc, XRR 060729, XRR 060904B, XRR 090618, XRR 091127/SN 2009nz, XRR 101219B/SN 2010ma, XRR 101225A, XRR 111228A, XRR 120422A/SN 2012bz, XRR 120714B/SN 2012eb, XRR 120729A, XRR 130215A/SN 2103ez, XRR 130831A/SN 2013fu, and XRR 150818A), and 3 C-GRBs (GRB 080319B, GRB 111209A/SN 2011kl, and GRB 130427A/SN 2013cq). Our statistical results are shown in Table 4. It seems that XRFs and XRRs are more favorable to link with SN events than C-GRBs.

\subsection{Host Galaxy Properties}

The host galaxies of XRFs were investigated in the work of Bloom et al. (2003). Here, we investigate the host galaxy properties for the XRFs, XRRs, and C-GRBs in the BAT3 catalog. We pay attention to several parameters of GRB host galaxies from the GRB Host Studies (GHostS) database $\mathrm{f}^{5}$. The physical quantities of GRB host galaxy are stellar mass $\left(M^{*}\right)$, metallicity $(Z)$, and star formation rate (SFR). The distributions of $M^{*}$ for XRFs, XRRs, and C-GRBs are shown in Figure 14. This Figure includes 6 XRFs, 17 XRRs, and 28 C-GRBs. We cannot find the significant differences among XRFs, XRRs, and C-GRBs, as we estimate the K-S test probabilities between XRFs and XRRs $(P=0.67)$, XRRs and CGRBs $(P=0.30)$, and XRFs and C-GRBs $(P=0.28)$. The distributions of metallicity $Z$ for XRFs, XRRs, and C-GRBs are shown in Figure 15. We obtain metallicity values of 5 XRFs, $6 \mathrm{XRRs}$, and $13 \mathrm{C}-\mathrm{GRBs}$. It is hard to distinguish the differences among XRFs, XRRs, and C-GRBs. The K-S test probabilities between XRFs and XRRs, between XRRs and C-GRBs, between XRFs and C-GRBs are $P=0.97, P=0.44$, and $P=0.90$, respectively. The distributions of SFR are shown in Figure 16. 4 XRFs, 11 XRRs, and 22 C-GRBs are included. The K-S test probabilities are $P=0.27$ (between XRFs and XRRs), $P=0.09$ (between XRRs and C-GRBs), and $P=0.03$ (between XRFs and C-GRBs). Therefore, it seems that there is an SFR difference between XRFs and C-GRBs. Here, we also note that only four XRFs have SFR values. This limitation prevents us for the further investigation.

GRB host galaxies are usually considered to be low-mass, low-metallicity, and starforming galaxies (Christensen et al. 2004; Fynbo et al. 2009; Savaglio et al. 2009). However, we see that some GRBs are hosted in massive and/or high-metallicity galaxies (e.g.,

\footnotetext{
${ }^{5}$ see the webpage http://www.grbhosts.org/. We note that GRB 120422 is classified as XRF in GhostS, but it is identified as XRR in this paper.
} 
Hashimoto et al. 2015). Mao (2010) proposed a possible redshift evolution of GRB host galaxies from the theoretical point of view. From the observational point of view, one survey of Swift-GRB host galaxy has recently been performed (Perley et al. 2016). We hope that more GRB host properties of XRFs, XRRs, and C-GRBs can be explored for the further statistical analysis in the future.

\subsection{Observational Biases and Selection Effects}

We should mention some selection effects and observational biases that may affect on our statistical results. First, the GRB prompt emission spectrum is usually fitted by the Band function (Band et al. 1993). However, the detection energy range of Swift-BAT is $15-350 \mathrm{keV}$, such that the spectral fitting is performed in the narrow energy range. The

$E_{\text {peak }}^{o b s}$ determination is from the cutoff power-law spectral model. Thus, the $E_{\text {peak }}^{\text {obs }}$ values in this sample might be different from those obtained from other space telescope detections with a wide energy range. Because GRB detections in a large energy range with a low energy threshold are required to accurately measure the $E_{\text {peak }}^{o b s}$ numbers, some future sensitive telescopes, such as the Space Variable Objects Monitor (SVOM) and the Einstein Probe (EP), are expected (Yuan et al. 2017). Second, GRB redshift values are determined by the spectral observations in the optical band. Thus, we cannot ignore the fact that many XRFs/XRRs have no redshift determinations. Hence, the XRF/XRR quantities related to the redshift cannot be determined. One incomplete sample may have bias on the redshift distribution (e.g., Fiore et al. 2007). Third, the observations for GRB host galaxies are also complicated. The detection of the high-redshift GRB host galaxies is one challenge. For example, Basa et al. (2012) performed the host galaxy search for three GRBs with $z>5$ using the Hubble Space Telescope, and they did not find any evidence of high-redshift GRB hosts. Although Mao et al. (2010) presented the possible redshift evolution of GRB host properties, the GRB redshift distribution with the cosmic star formation has some biased effects (e.g., Dainotti et al. 2015).

\section{Summary}

We present a comprehensively statistical analysis to study the XRF/XRR properties in the Swift-BAT3 catalog. We have obtained 81 XRFs and 540 XRRs in our sample. We have analyzed the properties of $\gamma$-ray prompt emission, X-ray emission, X-ray light curve type, association with SNe, and host galaxy properties for XRFs, XRRs, and C-GRBs. We list the major findings as follows: (1) Most XRFs/XRRs have low values of $E_{\text {peak }}^{o b s}$. We 
confirm that XRFs/XRRs mainly release their energy in the X-ray band, and they are lowluminosity GRBs. (2) Most XRFs/XRRs are long-duration GRBs. (3) XRFs, XRRs, and C-GRBs follow the same $E_{X, i s o}-E_{\gamma, i s o}-E_{p e a k, z}$ correlations. (4) We do not find any differences of redshift distributions among XRFs, XRRs, and C-GRBs in our sample. (5) XRFs seem to favor the association with SN explosions. (6) We find marginal but interesting evidence that different SFRs are shown between XRRs/XRFs and C-GRBs.

Although we see some differences between XRFs/XRRs and C-GRBs in some correlation studies and statistic results, we notice that the properties of XRFs, XRRs, and C-GRBs do not show a sharp difference. We confirm that XRFs and XRRs are belong to GRBs. However, the physical origin of XRF/XRR is still unclear. The jet off-axis effect is traditionally applied to explain the observational phenomena of XRFs/XRRs. The constraints of the jet beaming and the opening angle were already proposed (Rhoads 1999; Frail et al. 2001). However, it is not the case that each GRB with the jet off-axis is XRF/XRR. For example, GRB 080710 with the observational evidence of the jet off-axis (Krühler et al. 2009) is classified as C-GRB in this paper. On the other hand, the direct measurements to identify the jet off-axis evidence cannot be performed for each GRB. The jet beaming angle statistics related to the study of $\mathrm{XRF} / \mathrm{XRR}$ is expected (Gao \& Dai 2010). It is suggested that the GRB thermal component in the X-ray band can be one possible reason to explain XRF/XRR energy release. As an example, GRB 090618, identified as XRR in this paper, had a detection of thermal X-ray emission by Swift X-ray telescope, and this XRR is associated with SN explosion (Cano et al. 2011; Page et al. 2011). Starling et al. (2012) presented 11 Swift-detected GRBs with optical SN explosions, and the thermal X-ray signatures were clearly identified. However, compared to the XRFs/XRRs listed in this paper, the observed GRBs with the thermal emission that have optical SN explosion evidence are still very rare. From some recent theoretical modeling analysis, thermal emission may regulate the GRB spectral peak energy (Beloborodov 2013). Photospheric models can reproduce the GRB thermal emission in the $\gamma$-ray band (Vurm et al. 2013). We expect further observational cases of the GRB thermal emissions, although most of XRR and XRF spectra are still nonthermal. Finally, the GRB detectors with wide energy range and low energy threshold are expected especially for the study of XRFs and XRRs in the future.

The work is financially supported by the Open Project Program of the Key Laboratory for the Structure and Evolution of Celestial Objects, Chinese Academy of Sciences (grant No. OP201501), the Scientific Research Foundation of the Education Department of Yunnan Province (grant No. 2014Y463), and the National Natural Science Foundation of China (11404103). J. M. is supported by the National Natural Science Foundation of China (11673062 and 11661161010), the Hundred Talent Program, the Major Program of the Chi- 
nese Academy of Sciences (KJZD-EW-M06), and the Oversea Talent Program of Yunnan Province. J.-M. B. is partly supported by the National Natural Science Foundation of China (11433004) and the Ministry of Science and Technology of China (2016YFA0400700).

\section{REFERENCES}

Amati, L., Frontera, F., in't Zand, J. J. M., et al. 2004, A\&A, 426, 415

Amati, L. 2006, MNRAS, 372, 233

Amati, L., Guidorzi, C., Frontera, F., et al. 2008, MNRAS, 391, 577

Amati, L., Frontera, F., Guidorzi, C. 2009, A\&A, 508, 173

Band, D., Matteson, J., Ford, L., et al. 1993, ApJ, 413, 281

Barraud, C., Olive, J.-F., Lestrade, J. R., et al. 2003, A\&A, 400, 1021B

Barraud, C., Daigne, F., Mochkovitch, R., Atteia, J. L. 2005, A\&A, 440, 809

Basa S., Cuby, J. G., Savaglio, S., et al. 2012, A\&A, 542, A103

Beloborodov, A. M. 2013, ApJ, 764, 157

Bernardini, M. G., Margutti, R., Zaninoni, E., \& Chincarini, G. 2012, MNRAS, 425, 1199

Bersier, D., Fruchter, A. S., Strolger, L.-G., et al. 2006, ApJ, 643, 284

Bloom, J. S., Fox, D., van Dokkum, P. G., Kulkarni, S. R., Berger, E., Djorgovski, S. G., \& Frail, D. A. 2003, ApJ, 599, 957

Bulter, N. A., Sakamoto, T., Suzuki, M., et al. 2005, ApJ, 621, 884

Cano, Z., Bersier, D., Guidorzi, C., et al. 2011, MNRAS, 413, 669

Cano, Z. 2013, MNRAS, 434, 1098

Cano, Z., Wang, S. Q., Dai, Z. G. \& Wu, X.-F. 2017, Advances in Astronomy, 8929054, review to the special issue on "GRB in Swift/Fermi Era and Beyond", arXiv:1604.03549

Christensen, L., Hjorth, J., \& Gorosabel, J. 2004, A\&A, 425, 913

de Ugarte Postigo, A., Fatkhullin, T. A., Jóhannesson, G., et al. 2007, A\&A, 462, L57 
Dainotti, M. G., Del Vecchio, R., Shigehiro, N., \& Capozziello, S. 2015, ApJ, 800, 31

D’Alessio, V., Piro, L., Rossi, E. M. 2006, A\&A, 460, 653

D’Avanzo, P., Salvaterra, R., Bernardini, M. G., et al. 2014, MNRAS, 442, 2342

Donaghy, T. Q. 2006, ApJ, 645, 436

Fiore, F., Guetta, D., Piranomonte, S., et al. 2007, A\&A, 470, 515

Fong, W., Berger, E., Chornock, R., et al. 2013, ApJ, 769, 56

Frail, D. A., Kulkarni, S. R., Sari, R., et al. 2001, ApJ, 562, L55

Fynbo, J. P. U., Jakobsson, P., Prochaska, J. X., et al. 2009, ApJS, 185, 526

Galli, A., \& Piro, L. 2006, A\&A, 455, 413

Gao, Y., \& Dai, Z.-G. 2010, RAA, 10, 142

Gendre, B., Galli, A., Piroi, L. 2007, A\&A, 465, L13

Granot, J., Ramirez-Ruiz, E., \& Perna, R. 2005, ApJ, 630, 1003

Guidorzi, C., Clemens, C., Kobayashi, S., et al. 2009, A\&A, 499, 439

Grupe,D., Nousek, J. A., Veres, P., et al. 2013, ApJS, 209, 20

Hashimoto J., Perley, D. A., Ohta, K., et al. 2015, ApJ, 806, 250

Hjorth, J. \& Bloom, J. S. 2012, The Gamma-Ray Burst-Supernova Connection, pp.169-190. Cambridge Univ. Press, Cambridge

Kippen, R. M., Woods, P. M., Heise, J., in't Zand, J. J. M., Briggs, M. S., \& Preece, R. D. 2003, AIPC, 662, 244

Krühler, T., Greiner, J., Afonso, P., et al. 2009, A\&A, 508, 593

Lamb, D. Q., Donaghy, T. Q., \& Graziani, C. 2005, ApJ, 620, 355

Levan, A. J., Osborne, J. P., Tanvir, N. R. et al. 2006, ApJ, 648, 1132

Liang, E. W., Lin, T. T., Lü J., Lu, R.-J., Zhang J., \& Zhang, B. 2015, ApJ, 813, 116

Lien, A., Sakamoto, T., Barthelmy, S. D., et al. 2016, ApJ, 829, 7

Mao, J. 2010, ApJ, 717, 140 
Margutti, R., Zaninoni, E., Bernardini, M. G., et al. 2013, MNRAS, 428, 729

Maselli, A., Melandri, A., Nava, L., et al. 2014, Science, 343, 48

Melandri, A., Pian, E., D'Elia, V., et al. 2014, A\&A, 567, A29

Mizuta, A., Yamasaki, T., Nagataki, S., \& Mineshige, S. 2006, ApJ, 651, 960

Page, K. L., \& Gehrels, N. 2008, GCN circ. 7638

Page, K. L., Starling, R. L. C., Fitzpatrick, G., et al. 2011, MNRAS, 416, 2078

Pe'er, A., Mészáros, P., Rees, M. J. et al., 2006, ApJ, 642, 995

Perley, D. A., Krühler, T., Schulze, S., et al. 2016, ApJ, 817, 7

Pian, E., Mazzali, P., Masetti, N., et al. 2006, Nature, 442, 1011

Ramirez-Ruiz, E. 2005, MNRAS, 363, L61

Rhoads, J. E. 1999, ApJ, 525, 737

Romano, P., Moretti1, A., Banat, P. L., et al. 2006, A\&A, 450, 59

Sakamoto, T., Lamb, D. Q., Kawai, N., et al. 2005, ApJ, 629, 311

Sakamoto, T., Barbier, L., Barthelmy, S. D., et al. 2006, ApJ, 636, L73

Sakamoto, T., Hullinger, D., Sato, G., et al. 2008, ApJ, 679, 570

Sakamoto, T., Barthelmy, S. D., Baumgartner, W. H., et al. 2011, ApJS, 192, 1

Salafia, O. S., Ghisellini, G., Pescalli, A., Ghirlanda, G., \& Nappo, F. 2016, MNRAS, 461, 3607

Savaglio, S., Glazebrook, K., \& Le Borgne, D. 2009, ApJ, 691, 182

Schady, P., Mason, K. O., Osborne, M. J., et al. 2006, ApJ, 643, 276

Starling, R. L. C., Page, K. L., Pe'er, A., et al. 2012, MNRAS, 427, 2950

Soderberg, A. M., Kulkarni, S. R., Fox, D. B., et al. 2005, ApJ, 627, 877

Tunnicliffe, R. L., Levan, A. J., Tanvir, N. R., et al. 2014, MNRAS, 437, 1495

Urata, Y., Huang, K., Yamazaki, R., \& Sakamoto, T. 2015, ApJ, 806, 222 
Vestrand, W. T., Wren, J. A., Panaitescu, A., et al. 2014, Science, 343, 38

Virgili, F., Liang, E.-W., \& Zhang, B. 2009, MNRAS, 392, 91

Vurm, I., Lyubarsky, Y., \& Piran, T. 2013, ApJ, 764, 143

Woosley, S. E., \& Bloom, J. S. 2006, ARA\&A, 44, 507

Xu, L., Wu, X. F., \& Dai, Z. G. 2005, ApJ, 634, 1155

Yamazaki, R., Ioka, K., \& Nakamura, T. 2002, ApJ, 571, L31

Yuan, W., Amati, L., Cannizzo, J. K. et al. 2017, Space Science Reviews, 202, 235

Zaninoni, E., Bernardini, M. G., Margutti, R., et al. 2016, MNRAS, 455, 1375 


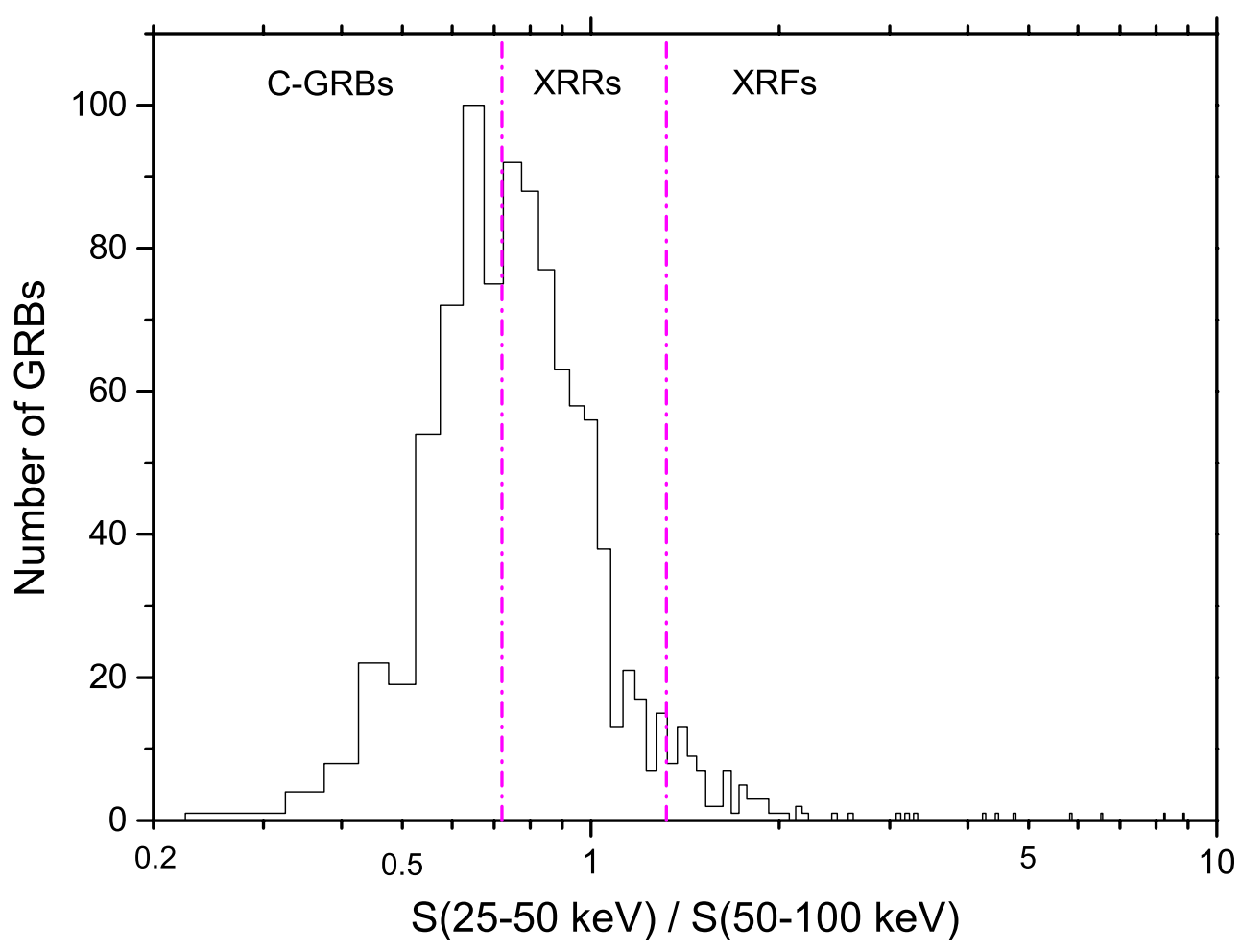

Fig. 1.- Distributions of the fluence ratio $S(25-50 \mathrm{keV}) / S(50-100 \mathrm{keV})$ of GRBs in SwiftBAT3 sample. The dashed lines show the distribution borders between C-GRBs and XRRs, and between XRRs and XRFs, respectively. Here, we list the sources with the fluence ratio larger than 3.0: XRF 050819 (the ratio is $3.08 \pm 0.99$ ), XRF 050406 (the ratio is $3.15 \pm 1.22$ ), $\mathrm{XRF} 060805 \mathrm{~A}$ (the ratio is $3.29 \pm 2.20$ ), XRF 080218B (the ratio is $4.24 \pm 3.08$ ), XRF 060428B (the ratio is $4.44 \pm 1.80$ ), $\mathrm{XRF} 080822 \mathrm{~B}$ (the ratio is $4.74 \pm 2.86$ ), $\mathrm{XRF} 050416 \mathrm{~A}$ (the ratio is $5.83 \pm 2.39$ ), $\mathrm{XRF} 160525 \mathrm{~A}$ (the ratio is $6.50 \pm 5.47$ ), $\mathrm{XRF} 061218$ (the ratio is $8.25 \pm 10.66$ ), and XRF 070126 (the ratio is $8.82 \pm 9.46$ ). 


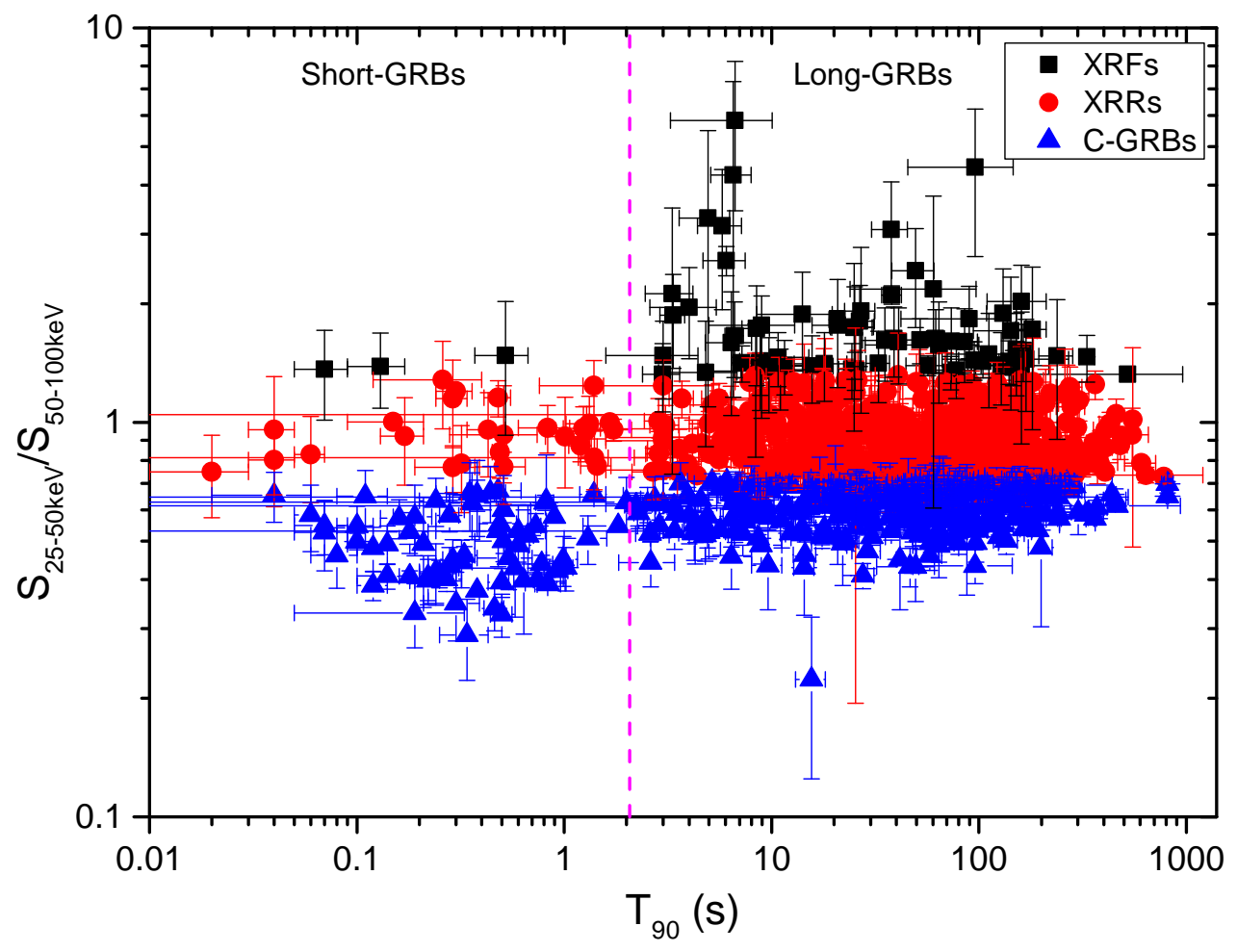

Fig. 2.- The fluence ratio of $S(25-50 \mathrm{keV}) / S(50-100 \mathrm{keV})$ vs. the duration $T_{90}$ of GRBs in the Swift-BAT3 sample. XRFs, XRRs, and C-GRBs are marked as black squares, red dots, and blue triangles, respectively. The dashed line shows the distribution border between long GRBs and short GRBs. 


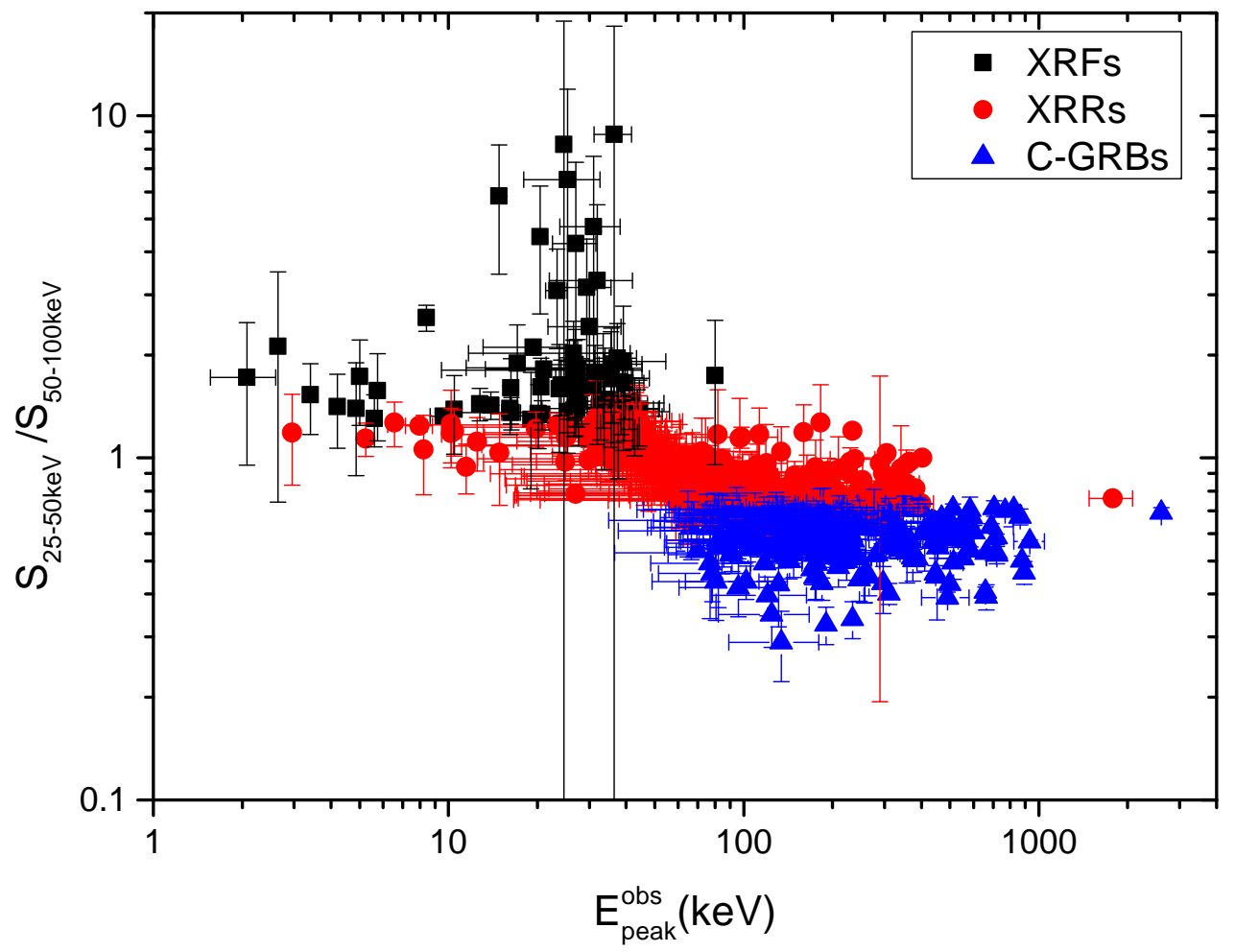

Fig. 3.- Fluence ratio of $S(25-50 \mathrm{keV}) / S(50-100 \mathrm{keV})$ versus the peak energy $E_{\text {peak }}^{\text {obs }}$ for XRFs, XRRs, and C-GRBs. XRFs, XRRs, and C-GRBs are marked as black squares, red dots, and blue triangles, respectively. XRF 061218 and XRF 070126 have large fluence ratio error bars, and they are not included in Figure 2 because they have no $T_{90}$ values. 


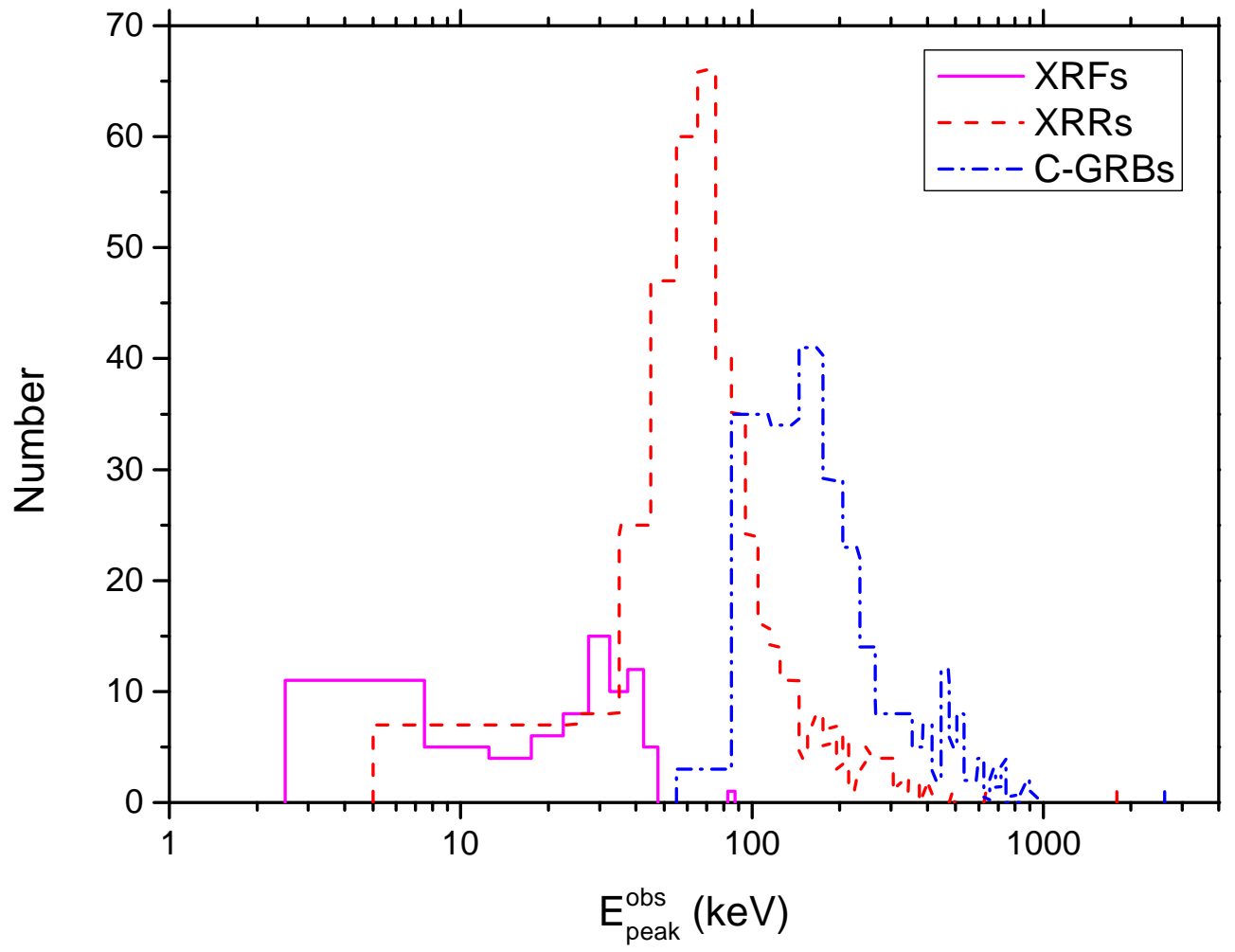

Fig. 4.- $E_{\text {peak }}^{o b s}$ distribution for XRFs, XRRs, and C-GRBs. XRFs, XRRs, and C-GRBs are marked as the pink solid line, the red dashed line, and the blue dash-dotted line, respectively. 


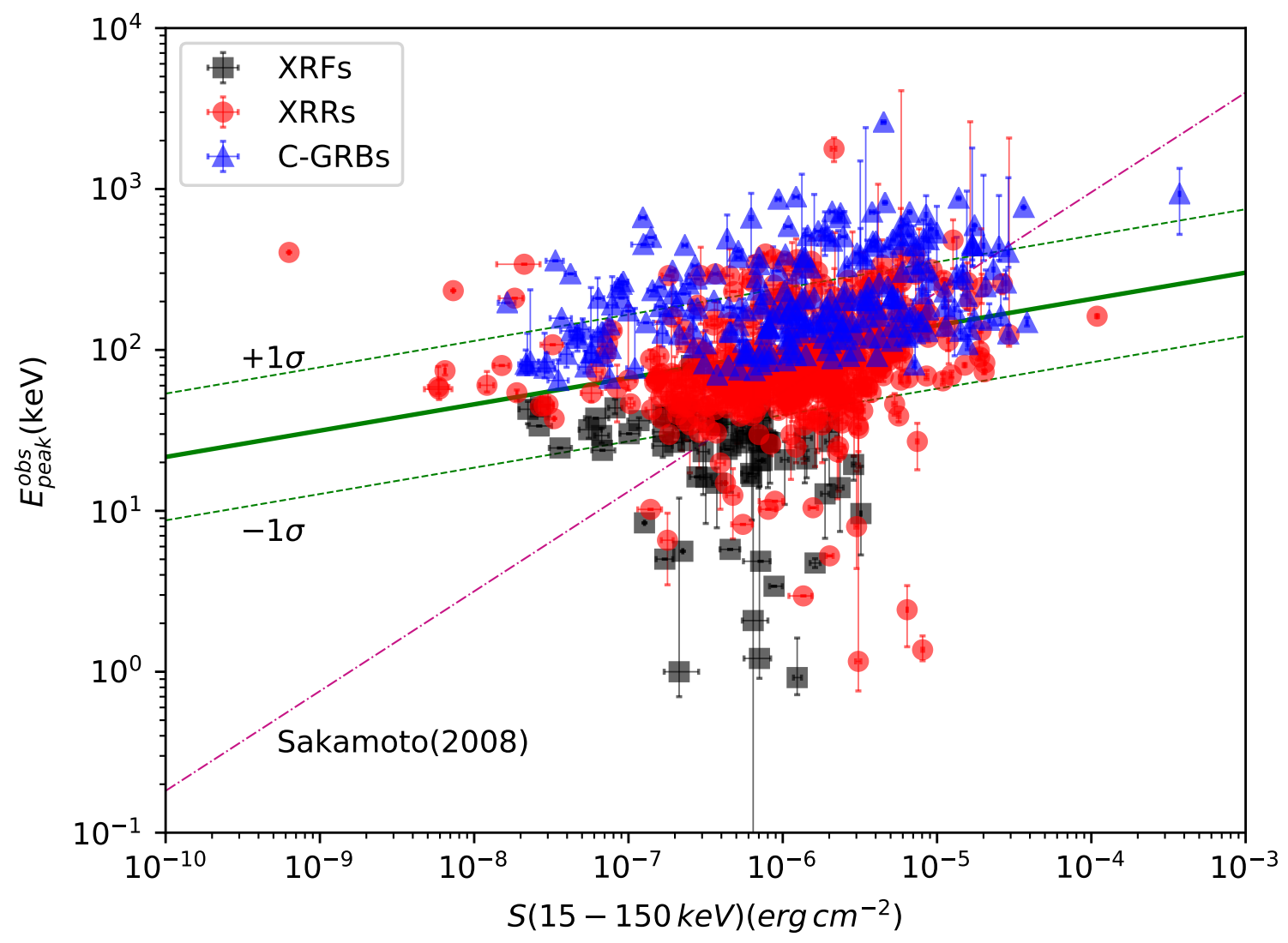

Fig. 5.- Relationship of the 15-150 keV fluence and the $E_{\text {peak }}^{o b s}$ for XRFs, XRRs, and C-GRBs. The green solid line is the best fit to the data with the function of $\log \left(E_{\text {peak }}^{o b s}\right)(\mathrm{keV})=(2.96 \pm$ $0.13)+(0.16 \pm 0.02) \log [S(15-150)] \mathrm{keV}$, and the green dashed lines are marked for the $1 \sigma$ regions. The extrinsic scatter $\sigma=0.39 \pm 0.01$. The pink dashed-dotted line is the best fit to the data without taking into account the errors reported by Sakamoto et al. (2008), and the function is $\log \left(E_{\text {peak }}^{\text {obs }}\right)(\mathrm{keV})=(5.46 \pm 0.25)+(0.62 \pm 0.14) \log [S(15-150) \mathrm{keV}]$. The XRFs, XRRs, and C-GRBs are marked as black squares, red dots, and blue triangles, respectively. 


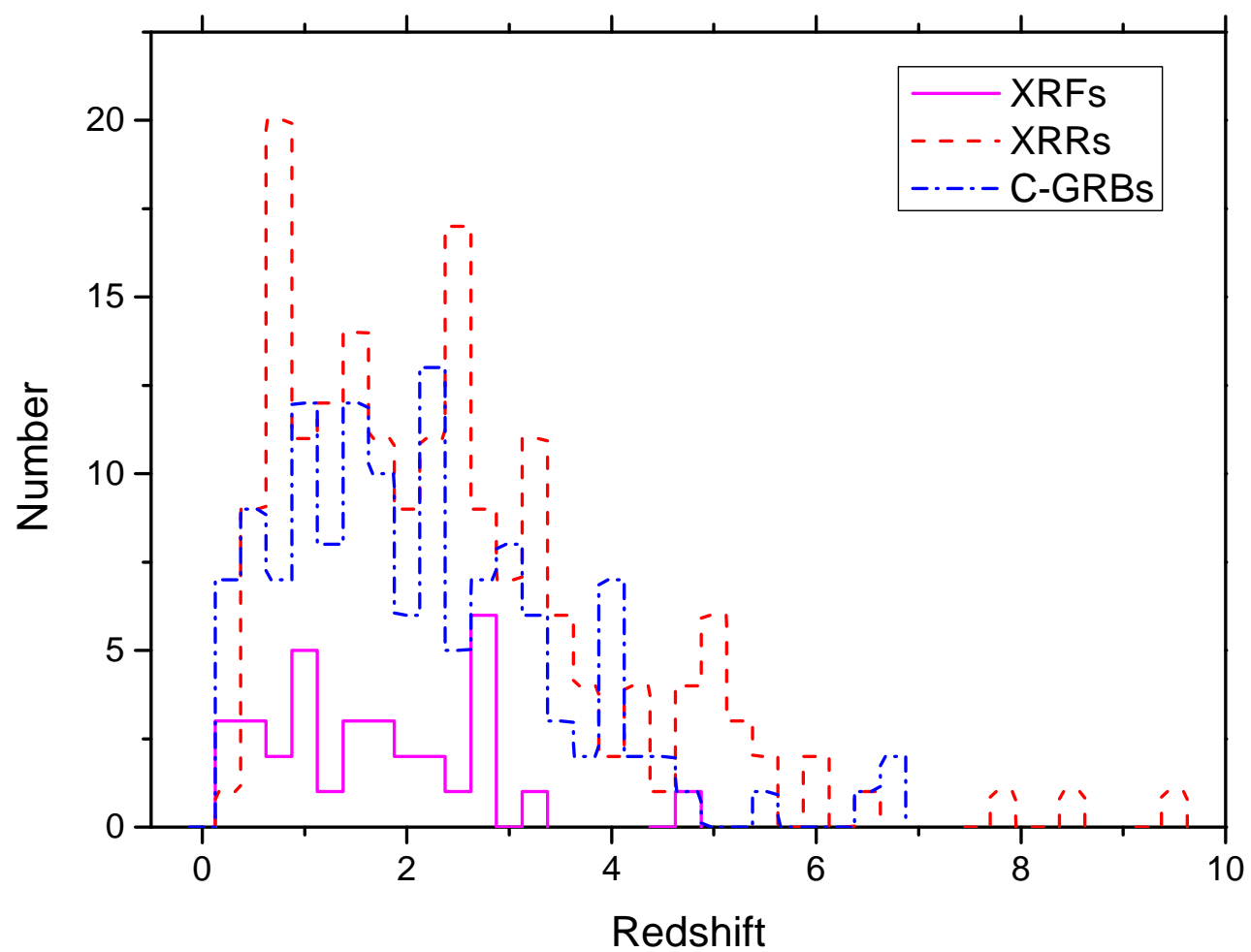

Fig. 6.- Redshift distribution of GRBs in the Swift-BAT3 sample. XRFs, XRRs, and CGRBs are marked as the pink solid line, the red dashed line, and the blue dashed-dotted line, respectively. 


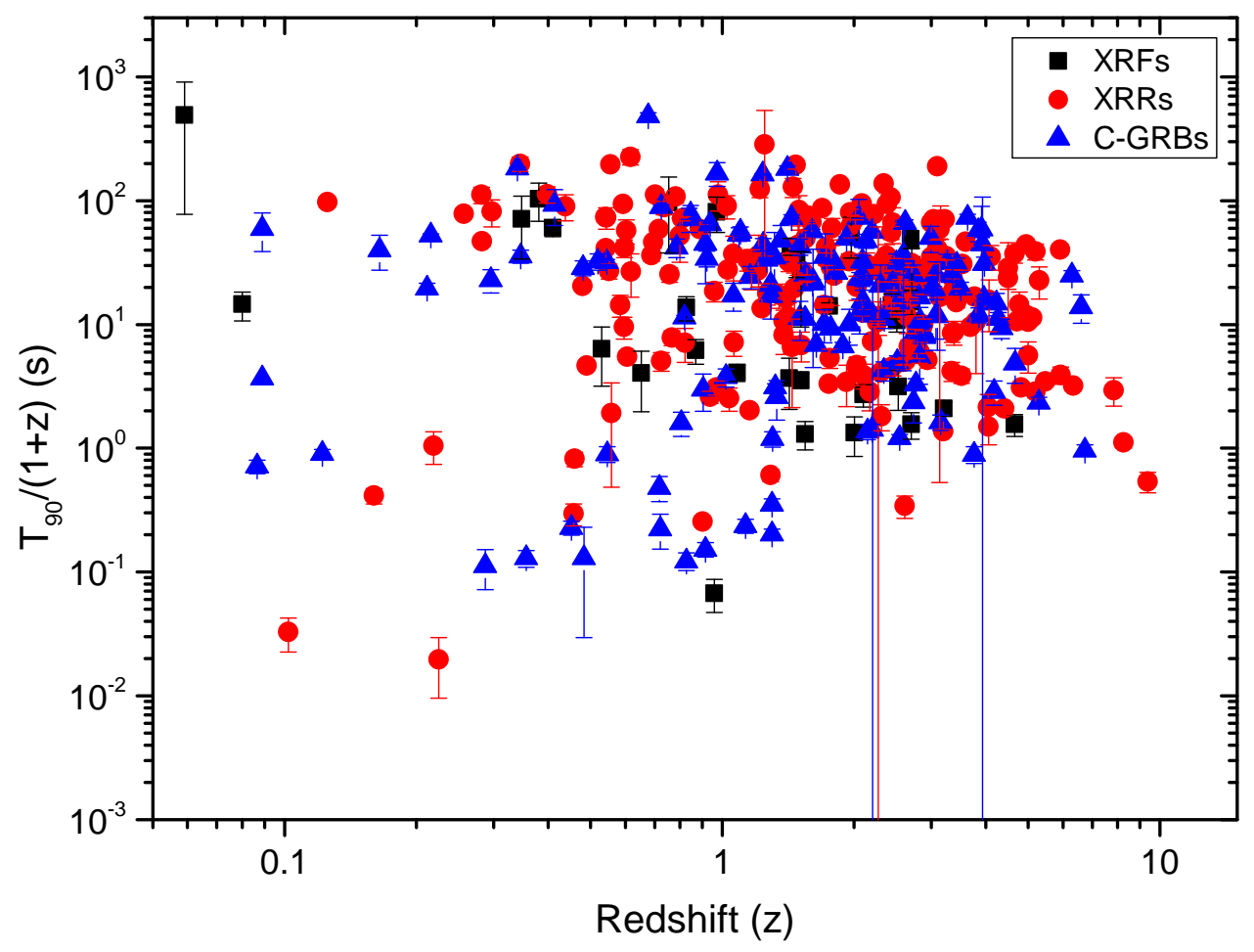

Fig. 7.- GRB duration $T_{90}$ vs. redshift. XRFs, XRRs, and C-GRBs are marked as black squares, red dots, and blue triangles, respectively. 


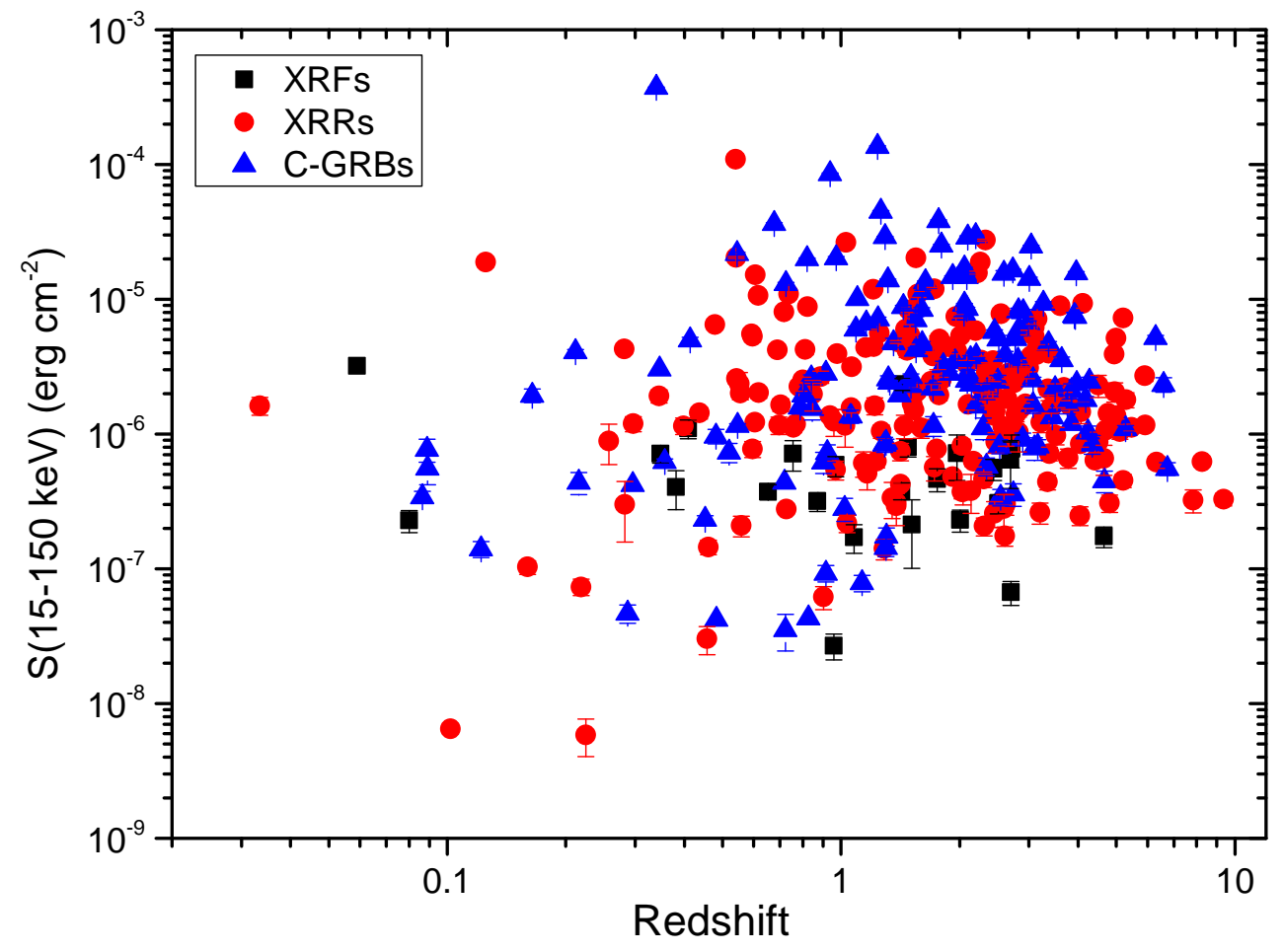

Fig. 8.- GRB fluence in the $15-150 \mathrm{keV}$ band vs. redshift. XRFs, XRRs, and C-GRBs are marked as black squares, red dots, and blue triangles, respectively. 


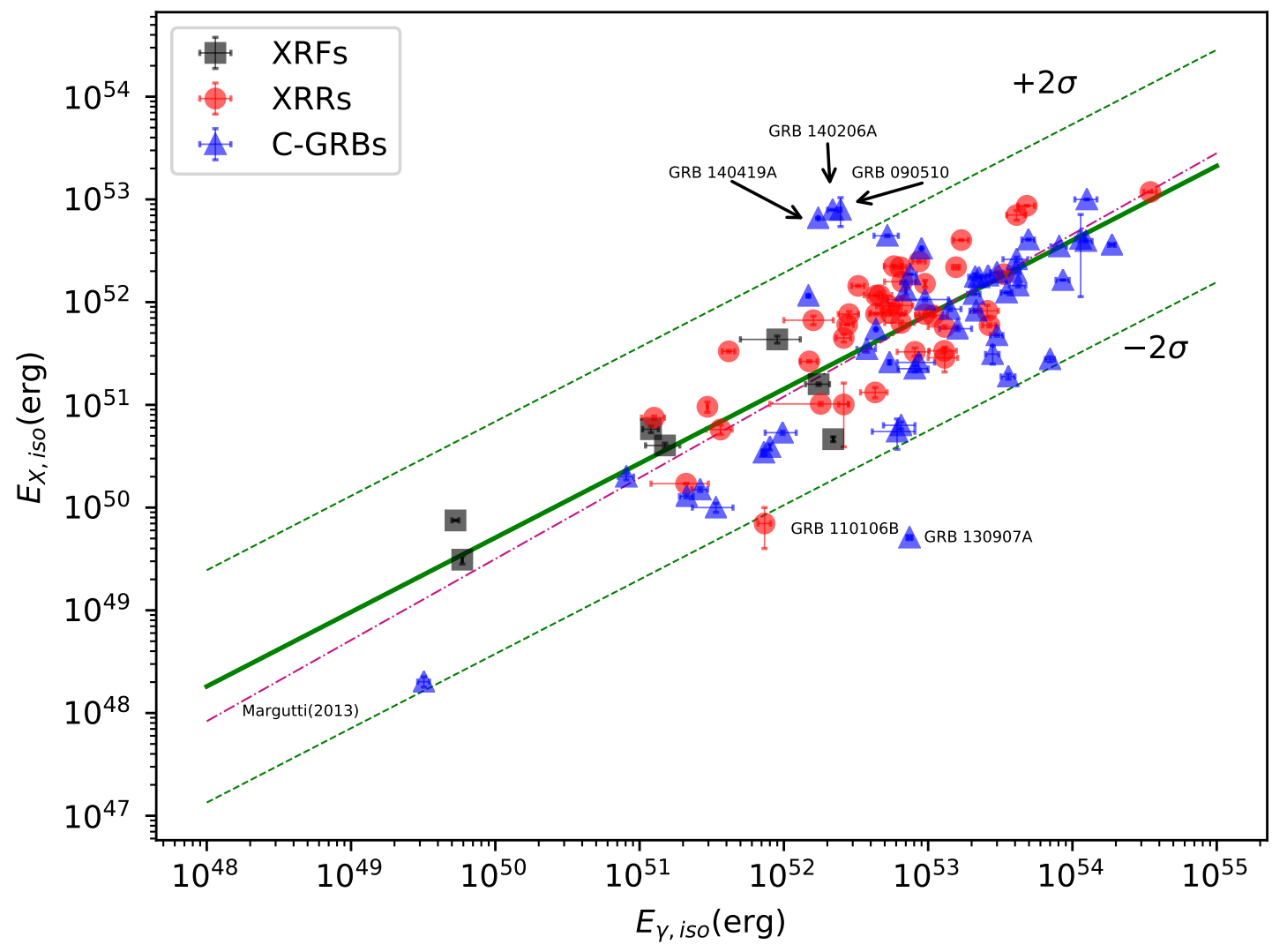

Fig. 9.- Correlation between $E_{X, \text { iso }}$ and $E_{\gamma, \text { iso }}$ in the Swift-BAT3 sample. The green solid line is the best fit with the function of $\left(E_{X, i s o}\right)=(13.31 \pm 2.73)+(0.73 \pm 0.05) \log \left(E_{\gamma, i s o}\right)$, and the extrinsic scatter is $\sigma=0.57 \pm 0.04$. The green dashed lines are marked for the $2 \sigma$ regions. The pink dashed-dotted line is the best-fitting function of $\log \left(E_{X, i s o}\right)=(10.0 \pm$ $20.6)+(0.79 \pm 0.01) \log \left(E_{\gamma, \text { iso }}\right)$ reported by Margutti et al.(2013). XRFs, XRRs, and C-GRBs are marked as black squares, red dots, and blue triangles, respectively. The GRBs labeled as outliers in the figure are L-GRBs, and the exception is short GRB 090510. 


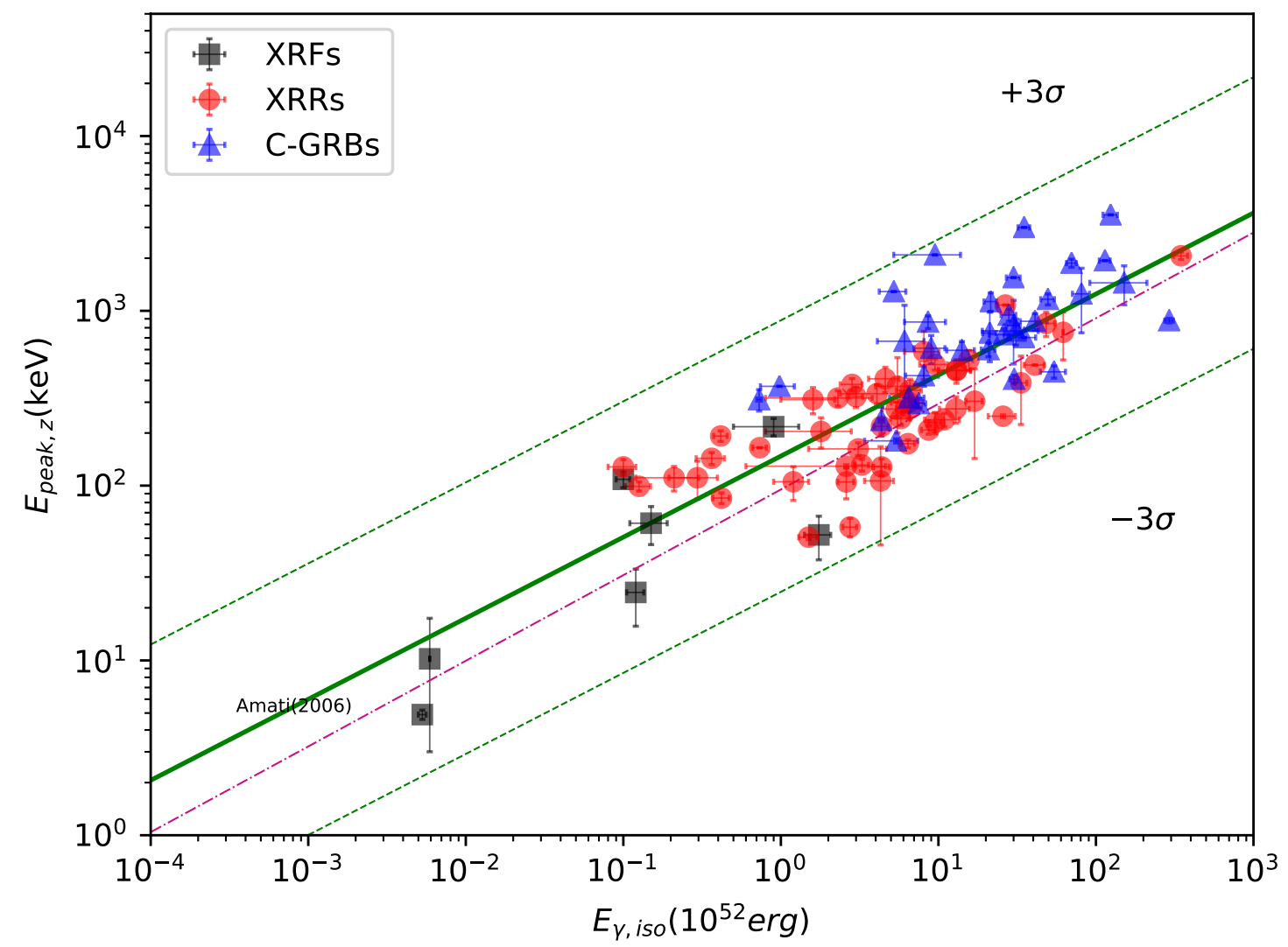

Fig. 10.- Correlation between GRB peak energy $E_{\text {peak,z }}$ in the rest frame and GRB isotropic-equivalent energy $E_{\gamma, \text { iso }}$. The green solid line is the best fit with the function of $\log \left(E_{\text {peak }, z}\right)(\mathrm{keV})=(2.17 \pm 0.04)+(0.46 \pm 0.03) \log \left[E_{\gamma, \text { iso }} /\left(10^{52} \mathrm{erg}\right)\right]$, and the extrinsic scatter is $\sigma=0.26 \pm 0.02$. and the green dashed lines are marked for the $3 \sigma$ regions. The pink dashed-dotted line is the best fit with the function of $E_{\text {peak }, z}=95 \mathrm{keV}\left(E_{\gamma, \text { iso }} / 10^{52} \mathrm{erg}\right)^{0.49}$ reported by Amati (2006). XRFs, XRRs, and C-GRBs are marked as black squares, red dots, and blue triangles, respectively. 


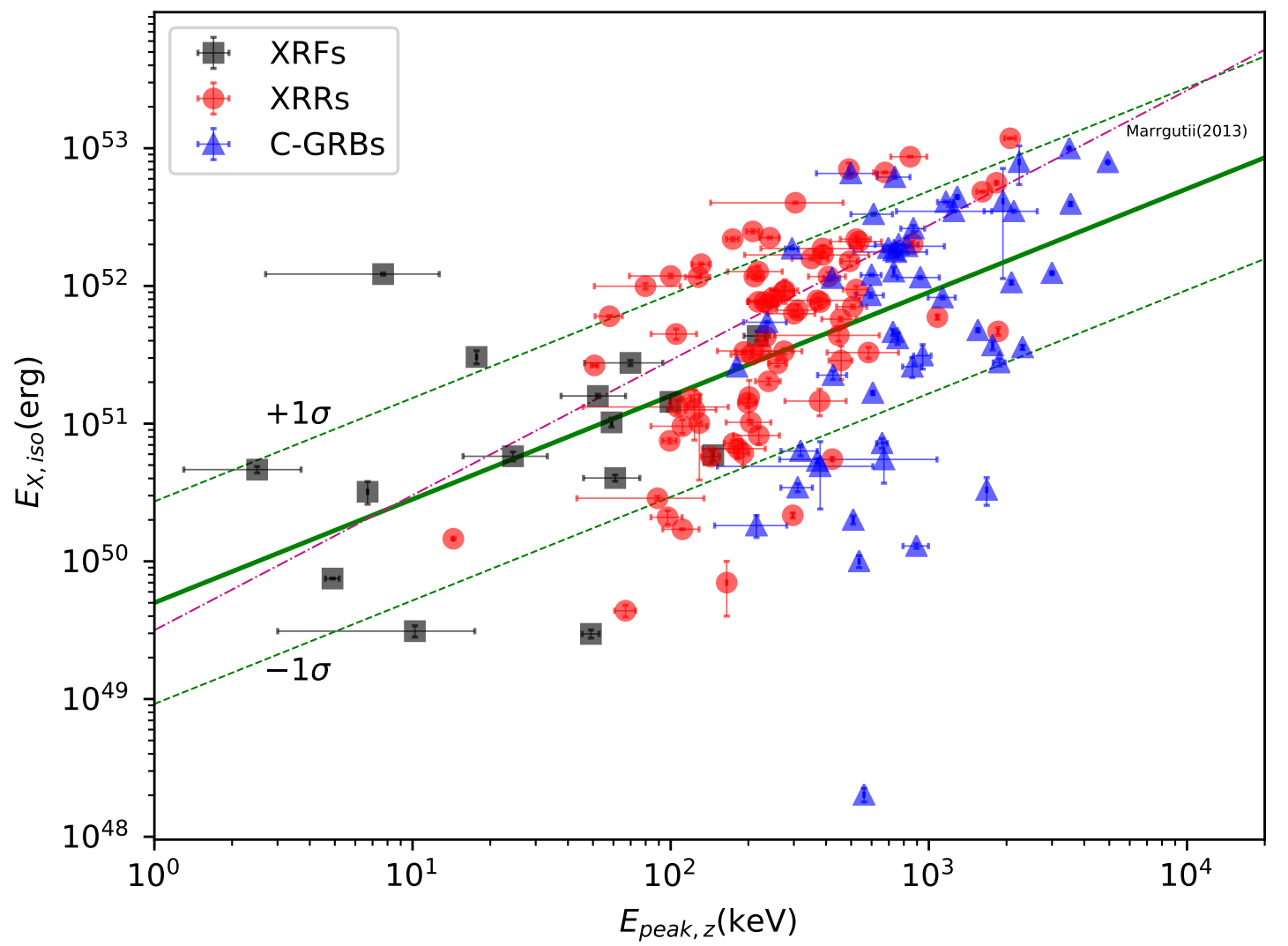

Fig. 11. - Correlation between GRB X-ray energy $E_{X, i s o}$ and GRB peak energy $E_{\text {peak,z }}$. The green solid line is the best fit with the function of $\log \left(E_{X, i s o}\right)=(49.69 \pm 0.27)+$ $(0.75 \pm 0.11) \log \left(E_{\text {peak }, z}\right)$, and the extrinsic scatter is $\sigma=0.73 \pm 0.04$. The green dashed lines are marked for the $1 \sigma$ regions. The pink dashed line is the best fit using the function of $\log \left(E_{X, i s o}\right)=(49.50 \pm 0.15)+(0.98 \pm 0.02) \log \left(E_{\text {peak,z }}\right)$ reported by Margutti et al. (2013). XRFs, XRRs, and C-GRBs are marked as black squares, red dots, and blue triangles, respectively. 


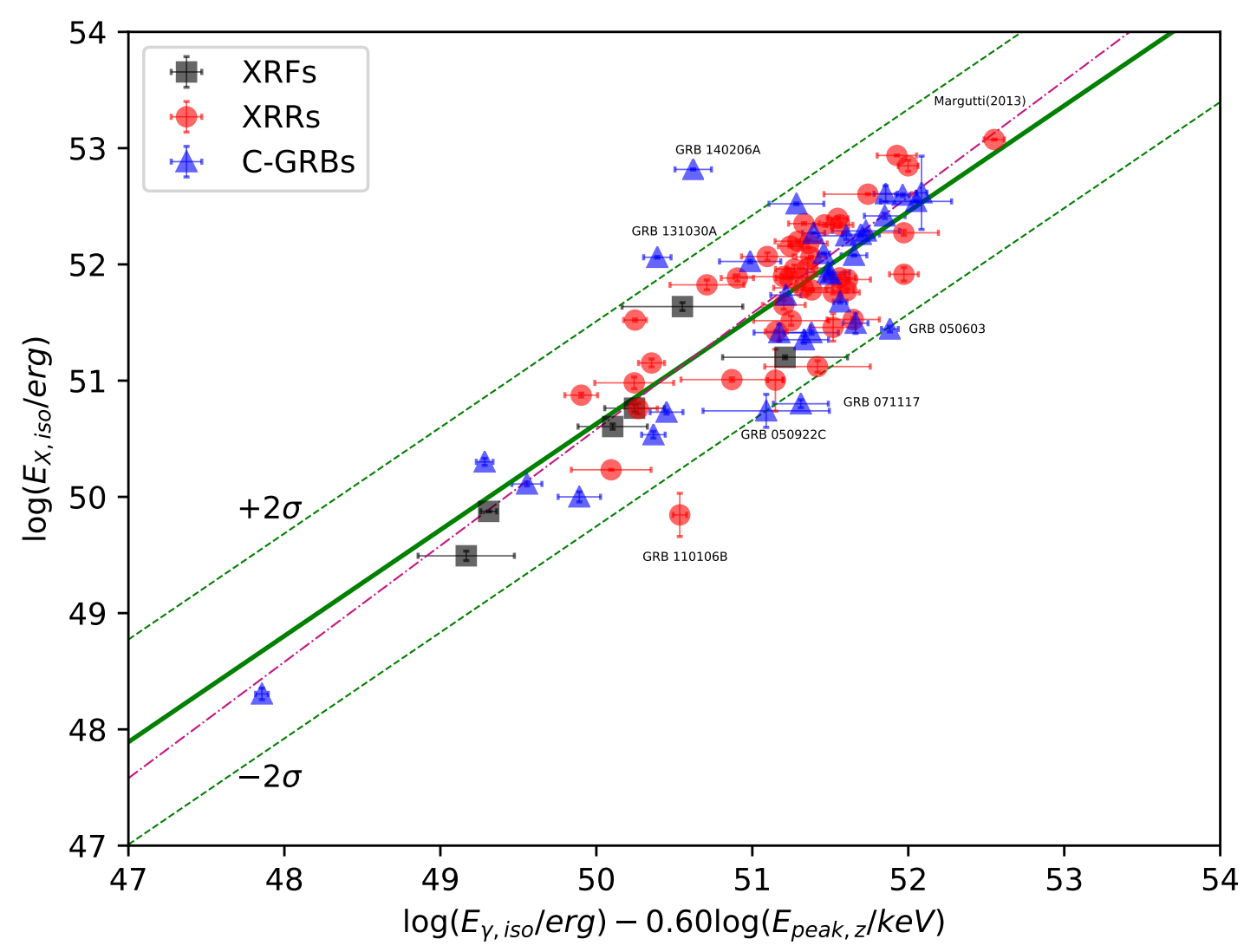

Fig. 12.- Correlation between $E_{X, i s o}$ and $E_{\gamma, i s o}-\left(E_{p e a k, z}\right)$. The green solid line is the best fit with the function of $\log \left(E_{X, i s o}\right)=(4.78 \pm 2.79)+(0.92 \pm 0.06)\left(\log E_{\gamma, \text { iso }}-0.6 \log \left(E_{\text {peak }}\right)\right.$ with the extrinsic scatter of $\sigma=0.44 \pm 0.04$. The green dashed lines are marked for the $2 \sigma$ regions. The pink dashed-dotted line is the best fit using the function of $\log \left(E_{X, i s o}\right)=$ $(0.58 \pm 0.25)+(1.00 \pm 0.06)\left(\log \left(E_{\gamma, i s o}\right)\right)-(0.60 \pm 0.10) \log \left(E_{\text {peak }}\right)$ reported by Margutti et al.(2013). XRFs, XRRs, and C-GRBs are marked as black squares, red dots, and blue triangles, respectively. All the GRBs labeled at outliers in the figure are L-GRBs. 


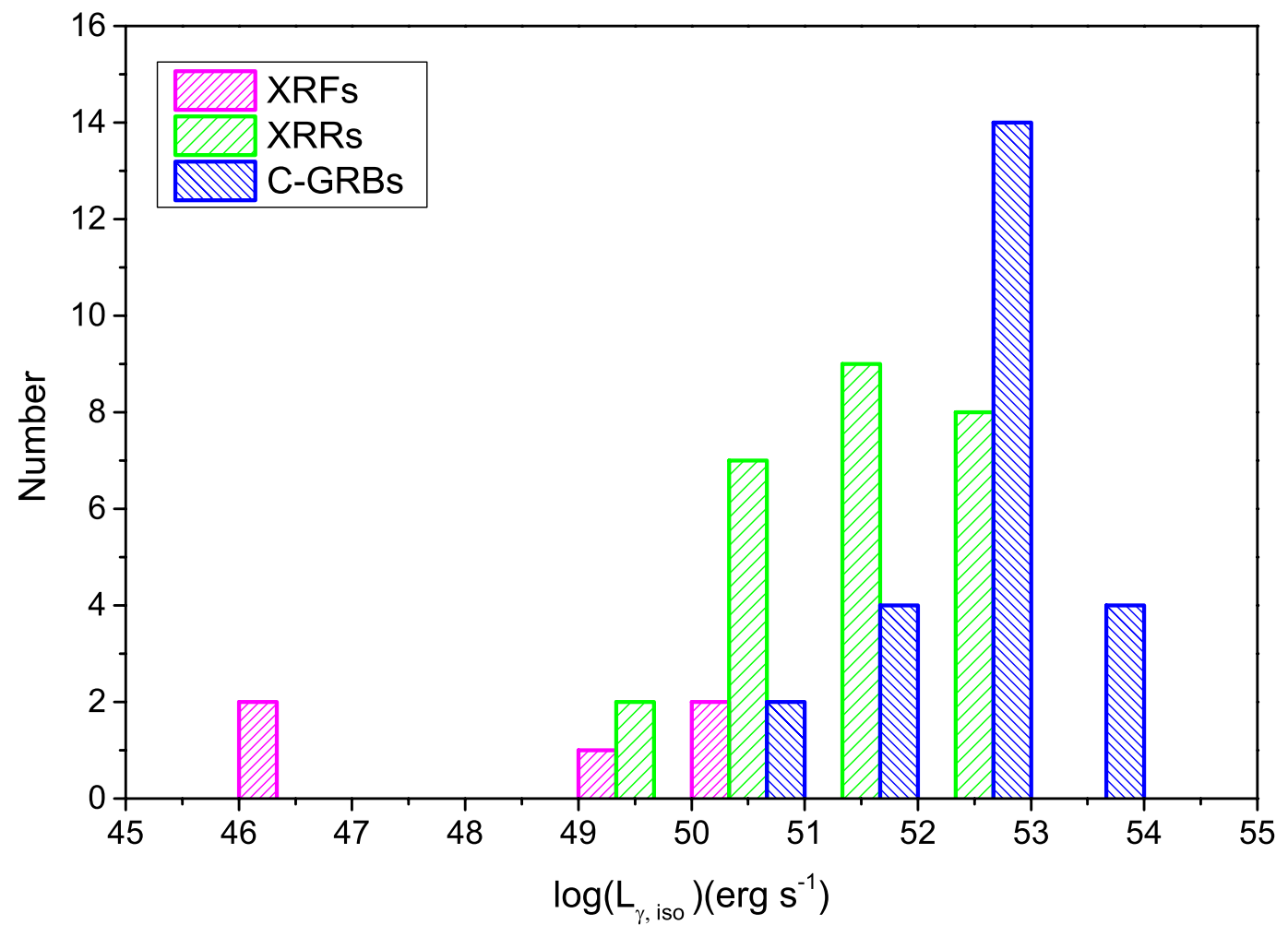

Fig. 13.- GRB isotropic luminosity $L_{\gamma, \text { iso }}$ distribution for XRF, XRR, and C-GRB classes. XRFs, XRRs, and C-GRBs are marked as pink, green, and blue, respectively. 


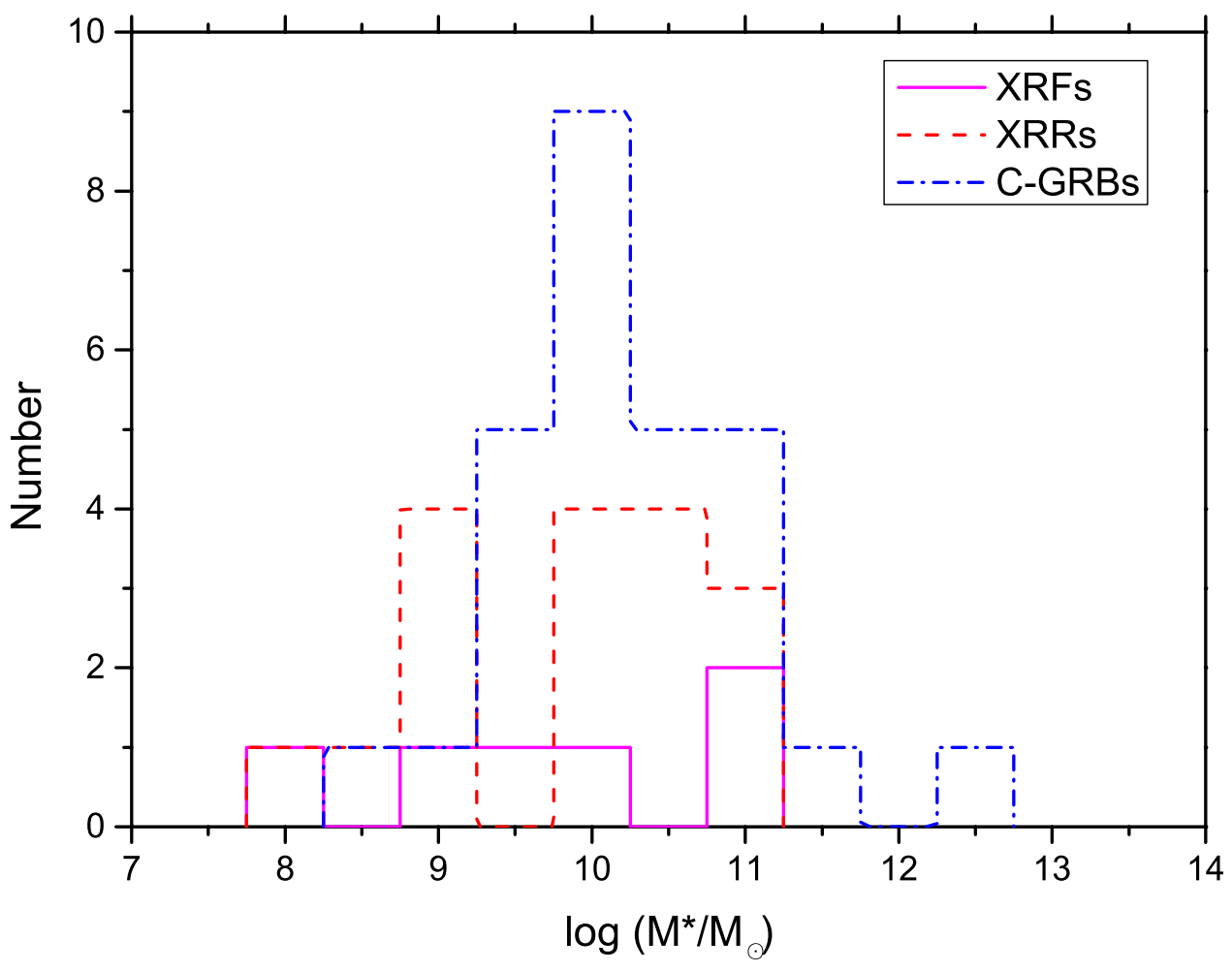

Fig. 14.- Distribution of stellar mass $\left(M^{*}\right)$ in GRB host galaxy for XRFs, XRRs, and C-GRBs. XRFs, XRRs, and C-GRBs are marked as the pink solid line, the red dashed line, and the blue dashed-dotted line, respectively. 


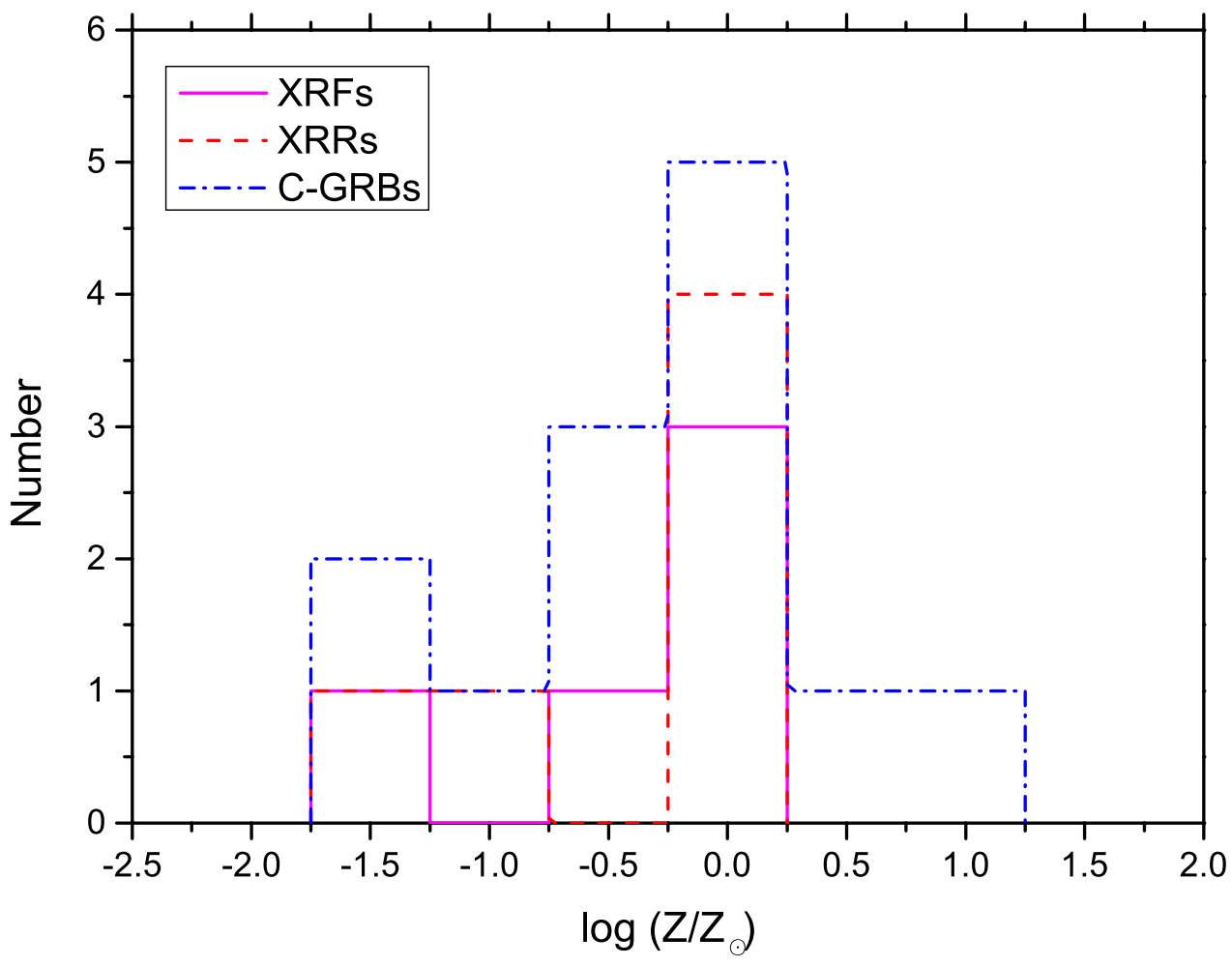

Fig. 15.- Distribution of metallicity $(Z)$ in GRB host galaxy for the XRFs, XRRs, and C-GRBs. XRFs, XRRs, and C-GRBs are marked as the pink solid line, the red dashed line, and the blue dashed-dotted line, respectively. 


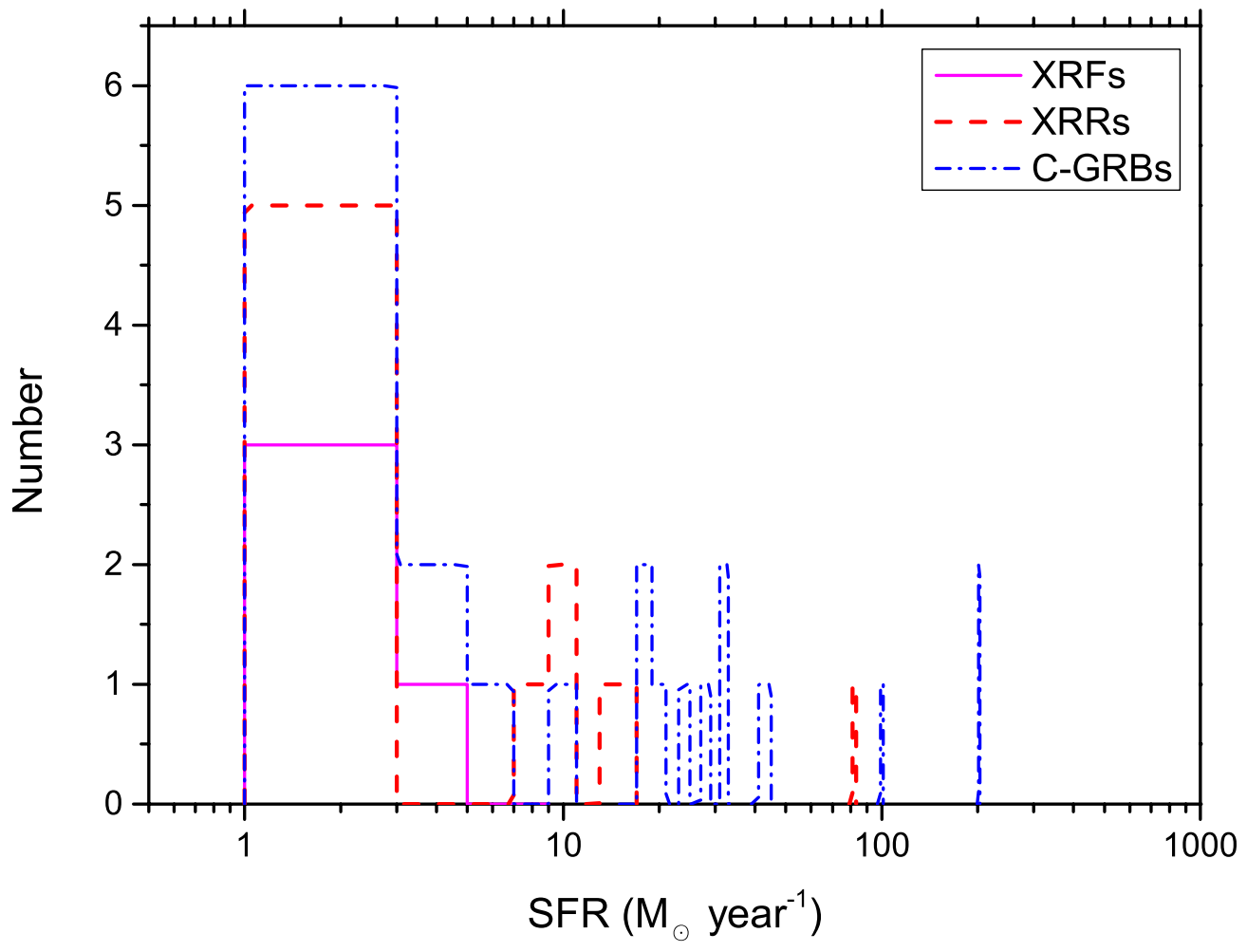

Fig. 16.- Distribution of star formation rate (SFR) in GRB host galaxy for XRFs, XRRs, and C-GRBs. XRFs, XRRs, and C-GRBs are marked as the pink solid line, the red dashed line, and the blue dashed-dotted line, respectively. 
Table 1. Statistical Results of XRF, XRR, and C-GRB in the Swift-BAT3 GRB catalog

\begin{tabular}{lcccc}
\hline \hline \multicolumn{1}{c}{ Class } & Total Number [fraction] & L-GRB Number [fraction] & S-GRB Number [fraction] & Unknown $^{b}[$ fraction] \\
\hline XRF & $81[(7.3 \pm 0.8) \%]$ & $70[(86.4 \pm 10.3) \%]$ & $3[(3.7 \pm 2.1) \%]$ & $8[(9.9 \pm 3.5) \%]$ \\
XRR & $540[(48.9 \pm 2.1) \%]$ & $509[(94.3 \pm 4.2) \%]$ & $27[(5.0 \pm 1.0) \%]$ & $4[(0.7 \pm 0.4) \%]$ \\
C-GRB & $394[(35.7 \pm 1.8) \%]$ & $328[(83.2 \pm 4.6) \%]$ & $65[(16.5 \pm 2.0) \%]$ & $1[(0.3 \pm 0.3) \%]$ \\
Unknown $^{a}$ & $89[(8.1 \pm 0.9) \%]$ & - & - & - \\
\hline
\end{tabular}

Note. - "a" indicates that these sources have no Swift-XRT observations, and we do not clarify them as XRFs, XRRs, or C-GRBs; "b" indicates that these sources have no $T_{90}$ numbers, and we cannot judge whether they belong to long-duration GRB or short-duration GRB subclass. 
Table 2. XRF and XRR samples

\begin{tabular}{|c|c|c|c|c|c|c|c|c|}
\hline GRB Name & $\mathrm{z}$ & $\begin{array}{r}T_{90} \\
(\mathrm{~s})\end{array}$ & $\alpha_{P L}$ & $\alpha_{C P L}$ & $\begin{array}{c}S(15-150 \mathrm{keV}) \\
\left(10^{-8} \mathrm{erg} \mathrm{cm}^{-2}\right)\end{array}$ & SR & $\begin{array}{c}E_{\text {peak }}^{o b s} \\
(\mathrm{keV})\end{array}$ & Ref \\
\hline XRF 050215B & 2.52 & 11.04 & $-2.25 \pm 0.12$ & -1.95 & 22.5 & $1.30 \pm 0.23$ & 5.6 & Lie16 \\
\hline XRF 050406 & 2.7 & 5.78 & $-2.40 \pm 0.17$ & $0.42_{-0.89}^{+1.50}$ & $6.7 \pm 0.7$ & $3.15 \pm 1.22$ & $29.4_{-3.0}^{+3.1}$ & Lie16 \\
\hline XRF 050416A & 0.6528 & 6.67 & $-3.17 \pm 0.14$ & $-0.82_{-0.77}^{+1.08}$ & $37.4 \pm 2.4$ & $5.83 \pm 2.39$ & $14.8_{-7.0}^{+3.6}$ & Lie16 \\
\hline XRF 050714B & 2.438 & 49.36 & $-2.40 \pm 0.17$ & $-0.01_{-0.72}^{+1.04}$ & $55.0 \pm 4.9$ & $2.42 \pm 0.68$ & $30.1_{-5.2}^{+3.0}$ & Lie16 \\
\hline XRF 050815 & - & 2.99 & $-1.82 \pm 0.12$ & $0.40_{-0.67}^{+0.86}$ & $8.2 \pm 0.8$ & $1.32 \pm 0.26$ & $43.6_{-3.5}^{+5.8}$ & Lie16 \\
\hline XRF 050819 & 2.5043 & 37.72 & $-2.64 \pm 0.14$ & $-0.49_{-0.65}^{+1.04}$ & $30.5 \pm 2.4$ & $3.08 \pm 0.99$ & $23.3_{-10.7}^{+3.0}$ & Lie16 \\
\hline XRF 050822 & 1.434 & 104.29 & $-2.33 \pm 0.07$ & $-1.81_{-0.10}^{+0.36}$ & $234.8 \pm 11.5$ & $1.43 \pm 0.13$ & $13.9_{-6.5}^{+7.4}$ & Lie16 \\
\hline XRF 050824 & 0.8278 & 25.01 & $-2.79 \pm 0.18$ & -1.00 & $25.6 \pm 2.5$ & $1.74 \pm 0.79$ & 80.0 & Lie16 \\
\hline XRF 051109B & 0.08 & 15.70 & $-1.98 \pm 0.12$ & $-0.36_{-0.56}^{+0.75}$ & $22.8 \pm 2.1$ & $1.39 \pm 0.27$ & $39.4_{-4.1}^{+5.9}$ & Lie16 \\
\hline XRF 060218 & 0.03342 & - & $-2.18 \pm 0.10$ & $-1.65_{-0.18}^{+0.48}$ & $162.2 \pm 12.1$ & $1.37 \pm 0.18$ & $4.7 \pm 0.3$ & Ama08 \\
\hline XRF 060219 & - & 62.04 & $-2.53 \pm 0.16$ & $\begin{array}{r}-0.97_{-0.52}^{+0.18} \\
-0.98\end{array}$ & $43.6 \pm 4.1$ & $1.63 \pm 0.30$ & 24.1 & Lie16 \\
\hline XRF 060428B & 0.348 & 96.00 & $-2.78 \pm 0.12$ & $-0.41_{-0.79}^{+1.21}$ & $71.2 \pm 5.1$ & $4.44 \pm 1.80$ & 20.4 & Lie16 \\
\hline XRF 060512 & 2.1 & 8.40 & $-2.36 \pm 0.15$ & $-1.58_{-0.21}^{+1.08}$ & - & $1.30 \pm 0.48$ & 19.0 & Lie16 \\
\hline XRF 060516 & - & 128.00 & $-2.04 \pm 0.12$ & $\begin{array}{r}-0.80_{-0.79}^{+0.81} \\
\end{array}$ & $74.6 \pm 7.4$ & $1.40 \pm 0.29$ & $36.3_{-8.8}^{+6.8}$ & Lie16 \\
\hline XRF 060805A & - & 4.93 & $-2.11 \pm 0.19$ & $\begin{array}{l}0.49_{-1.13}^{+3.69} \\
\end{array}$ & $5.5 \pm 0.9$ & $3.29 \pm 2.20$ & $31.9_{-3.7}^{+6.0}$ & Lie16 \\
\hline XRF 060923B & 1.51 & 8.95 & $-2.47 \pm 0.12$ & $\begin{array}{l}-1.81_{-0.09}^{+0.65} \\
+1\end{array}$ & - & $1.38 \pm 0.36$ & 10.5 & Lie16 \\
\hline XRF 060923C & - & 73.73 & $-2.24 \pm 0.11$ & $-1.13_{-0.44}^{+0.89}$ & $138.8 \pm 13.0$ & $1.60 \pm 0.35$ & $28.3_{-13.4}^{+4.6}$ & Lie16 \\
\hline XRF 060926 & 3.2086 & 8.82 & $-2.50 \pm 0.11$ & -1.93 & - & $1.41 \pm 0.34$ & 4.2 & Lie16 \\
\hline XRF 061027 & - & - & $-1.88 \pm 0.23$ & $0.14 \pm 1.02$ & $24.0 \pm 5.5$ & $1.46 \pm 0.25$ & 41.0 & Lie16 \\
\hline XRF 061210 & 0.4095 & 85.23 & $-1.55 \pm 0.14$ & $-1.56_{-0.13}^{+0.14}$ & $109.8 \pm 8.8$ & $1.60 \pm 0.34$ & - & - \\
\hline XRF 061218 & - & - & $-2.61 \pm 0.29$ & $0.87_{-1.42}^{+3.95}$ & $3.6 \pm 0.6$ & $8.25 \pm 10.66$ & 24.6 & Lie16 \\
\hline XRF 070126 & - & - & $-1.88 \pm 0.25$ & $2.39 \pm 0.50$ & $11.7 \pm 1.9$ & $8.82 \pm 9.46$ & $36.4_{-3.8}^{+1.5}$ & Lie16 \\
\hline XRF 070330 & - & 6.64 & $-1.88 \pm 0.25$ & $0.67_{-0.66}^{+1.04}$ & $16.7 \pm 1.4$ & $1.66 \pm 0.36$ & $39.6_{-2.5}^{+3.8}$ & Lie16 \\
\hline XRF 070419A & 0.9705 & 160.0 & $-2.37 \pm 0.12$ & $-0.84_{-0.53}^{+0.78}$ & $58.2 \pm 4.9$ & $2.02 \pm 0.48$ & $26.5_{-11.2}^{+3.6}$ & Lie16 \\
\hline XRF 070714A & - & 3.00 & $-2.56 \pm 0.10$ & $-1.97 \pm 0.46$ & - & $1.48 \pm 0.33$ & $1.8 \pm 0.7$ & Lie16 \\
\hline XRF $070721 \mathrm{~A}$ & - & 3.35 & $-2.35 \pm 0.18$ & $1.35_{-1.27}^{+3.80}$ & - & $1.87 \pm 0.50$ & $26.9_{-2.7}^{+2.8}$ & Lie16 \\
\hline XRF 071008 & - & 17.98 & $-2.14 \pm 0.13$ & $-1.11_{-0.52}^{+0.70}$ & $22.5 \pm 2.2$ & $1.41 \pm 0.28$ & 32.7 & Lie16 \\
\hline XRF 071031 & 2.6918 & 180.64 & $-2.35 \pm 0.13$ & $-1.99_{-0.01}^{+0.14}$ & $64.3 \pm 12.5$ & $1.72 \pm 0.76$ & $2.1_{-107.5}^{+187.8}$ & Lie16 \\
\hline XRF 071101 & - & 4.82 & $-2.00 \pm 0.22$ & $-0.82_{-0.66}^{+1.48}$ & $6.1 \pm 1.1$ & $1.34 \pm 0.47$ & $\begin{array}{l}-107.5 \\
37.7\end{array}$ & Lie16 \\
\hline XRF 071227 & 0.381 & 142.48 & $-2.01 \pm 0.18$ & $-0.23_{-0.87}^{+1.69}$ & $40.4 \pm 6.4$ & $1.71 \pm 0.63$ & $35.5 \pm 5.3$ & Lie16 \\
\hline XRF 080218B & - & 6.53 & $-2.53 \pm 0.14$ & $1.30_{-1.07}^{+2.43}$ & - & $4.24 \pm 3.08$ & $27.0_{-2.7}^{+1.8}$ & Lie16 \\
\hline XRF 080315 & - & - & $-2.18 \pm 0.27$ & 1.58 & $10.1 \pm 1.5$ & $53.78 \pm 143.02$ & 30.1 & Lie16 \\
\hline XRF 080319D & - & 26.94 & $-1.79 \pm 0.17$ & $0.49_{-1.15}^{+2.47}$ & $23.5 \pm 4.3$ & $1.92 \pm 0.86$ & $39.1 \pm 4.1$ & Lie16 \\
\hline XRF 080330 & 1.5119 & 60.36 & $-2.65 \pm 0.25$ & $-1.98_{-0.01}^{+0.56}$ & $21.3 \pm 5.6$ & $2.18 \pm 1.57$ & $1.0_{-0.3}^{+11.0}$ & Lie16 \\
\hline XRF 080515 & - & 20.86 & $-2.19 \pm 0.08$ & $\begin{array}{l}-0.39_{-0.48}^{+0.59} \\
\text { - }\end{array}$ & - & $1.76 \pm 0.54$ & $33.5_{-3.1}^{+2.3}$ & Lie16 \\
\hline XRF 080520 & 1.5457 & 3.32 & $-3.08 \pm 0.26$ & -1.90 & - & $2.12 \pm 1.38$ & 2.6 & Lie16 \\
\hline XRF 080822B & - & - & $-2.41 \pm 0.26$ & $2.00_{-1.35}^{+2.83}$ & $16.8 \pm 2.4$ & $4.74 \pm 2.86$ & $31.0_{-3.8}^{+3.4}$ & Lie16 \\
\hline XRF 081007 & 0.5295 & 9.73 & $-2.43 \pm 0.10$ & $-1.52_{-0.33}^{+0.55}$ & - & $1.35 \pm 0.29$ & $39.9 \pm 9.8$ & Ama09 \\
\hline XRF 090111 & - & 25.26 & $-2.28 \pm 0.08$ & $\begin{array}{r}-1.79_{-0.22}^{+0.38} \\
-0.38\end{array}$ & $63.0 \pm 3.6$ & $1.36 \pm 0.15$ & $16.4_{-7.6}^{+8.1}$ & Lie16 \\
\hline XRF 090205 & 4.6497 & 8.81 & $-2.01 \pm 0.11$ & $-0.39_{-0.56}^{+0.74}$ & $17.4 \pm 1.5$ & $1.43 \pm 0.36$ & $38.4_{-3.8}^{+5.0}$ & Lie16 \\
\hline XRF 090308A & - & 25.10 & $-2.34 \pm 0.17$ & $-1.09_{-0.76}^{+0.94}$ & $19.9 \pm 2.3$ & $1.74 \pm 0.46$ & 26.7 & Lie16 \\
\hline XRF 090417A & - & 0.07 & $-2.81 \pm 0.16$ & $0.39 \pm 0.76$ & $2.2 \pm 0.3$ & $1.36 \pm 0.35$ & $42.86_{-8.0}^{+5.1}$ & Lie16 \\
\hline XRF 090726 & 2.71 & 56.68 & $-2.29 \pm 0.10$ & $-1.30_{-0.41}^{+0.50}$ & $78.7 \pm 4.4$ & $1.39 \pm 0.08$ & 26.9 & Lie16 \\
\hline XRF 090807A & - & 146.35 & $-2.22 \pm 0.07$ & $\begin{array}{r}-0.41 \\
-1.48_{-0.27}^{+0.37}\end{array}$ & $199.8 \pm 10.9$ & $1.38 \pm 0.14$ & $27.0_{-12.5}^{+4.4}$ & Lie16 \\
\hline XRF $100212 \mathrm{~A}$ & - & 163.76 & $-2.24 \pm 0.10$ & $\begin{array}{r}-2.22_{-0.23}^{+0.09} \\
\text { + }\end{array}$ & $102.0 \pm 7.9$ & $1.52 \pm 0.25$ & - & - \\
\hline
\end{tabular}


Table 2-Continued

\begin{tabular}{|c|c|c|c|c|c|c|c|c|}
\hline GRB Name & $\mathrm{z}$ & $\begin{array}{l}T_{90} \\
(\mathrm{~s})\end{array}$ & $\alpha_{P L}$ & $\alpha_{C P L}$ & $\begin{array}{c}S(15-150 \mathrm{keV}) \\
\left(10^{-8} \mathrm{erg} \mathrm{cm}^{-2}\right)\end{array}$ & SR & $\begin{array}{l}E_{\text {peak }}^{o b s} \\
(\mathrm{keV})\end{array}$ & Ref \\
\hline XRF 100316D & 0.0591 & 521.88 & $-2.36 \pm 0.08$ & $-1.88_{-0.06}^{+0.37}$ & 321.0 & 1.33 & $9.6_{-4.3}^{+9.3}$ & Lie16 \\
\hline XRF 100324A & - & 6.40 & $-2.28 \pm 0.22$ & $-1.40_{-0.34}^{+1.34}$ & $6.8 \pm 1.2$ & $1.59 \pm 0.55$ & 23.8 & Lie16 \\
\hline XRF 100425A & 1.755 & 38.97 & $-2.44 \pm 0.16$ & $-0.88_{-0.67}^{+1.23}$ & $46.1 \pm 4.5$ & $1.63 \pm 0.33$ & $25.4_{-12.3}^{+4.7}$ & Lie16 \\
\hline XRF 110112A & - & 0.52 & $-2.12 \pm 0.23$ & $-0.89_{-0.61}^{+1.67}$ & $2.6 \pm 0.5$ & $1.48 \pm 0.55$ & 33.7 & Lie16 \\
\hline XRF $110312 \mathrm{~A}$ & - & 35.14 & $-2.33 \pm 0.13$ & $\begin{array}{r}-0.63_{-0.38}^{+0.73} \\
-1.53^{-0.3}\end{array}$ & 73.6 & $1.61 \pm 0.34$ & 20.6 & Lie16 \\
\hline XRF 110319A & - & 20.68 & $-2.55 \pm 0.04$ & $1.38 \pm 0.22$ & $143.2 \pm 3.6$ & $1.82 \pm 0.10$ & $21.0_{-5.0}^{+2.7}$ & Lie16 \\
\hline XRF $111018 \mathrm{~A}$ & - & 32.71 & $-2.18 \pm 0.14$ & $-2.16_{-0.03}^{+1.28}$ & $36.3 \pm 4.0$ & $1.41 \pm 0.29$ & - & - \\
\hline XRF 111029A & - & 7.20 & $-2.11 \pm 0.07$ & $\begin{array}{l}-0.77_{-0.33}^{+0.39} \\
\end{array}$ & $37.3 \pm 1.9$ & $1.42 \pm 0.15$ & $36.1_{-3.0}^{+2.7}$ & Lie16 \\
\hline XRF 111129A & 1.0796 & 8.48 & $-2.53 \pm 0.19$ & -1.91 & $17.2 \pm 2.1$ & $1.73 \pm 0.49$ & 5.0 & Lie16 \\
\hline XRF 120116A & - & 38.04 & $-2.69 \pm 0.04$ & $-1.31_{-0.20}^{+0.22}$ & $288.3 \pm 6.4$ & $2.11 \pm 0.11$ & $19.4_{-3.9}^{+2.4}$ & Lie16 \\
\hline XRF $120724 \mathrm{~A}$ & 1.48 & 77.92 & $-2.43 \pm 0.13$ & $-0.75_{-0.53}^{+0.80}$ & $80.1 \pm 6.2$ & $1.36 \pm 0.21$ & $26.7_{-12.8}^{+3.9}$ & Lie16 \\
\hline XRF $121108 \mathrm{~A}$ & - & 89.55 & $-2.29 \pm 0.10$ & $-1.01_{-0.48}^{+0.63}$ & $82.8 \pm 6.4$ & $1.83 \pm 0.38$ & $26.5_{-11.7}^{+3.4^{8}}$ & Lie16 \\
\hline XRF $121212 \mathrm{~A}$ & - & 6.06 & $-2.50 \pm 0.19$ & -1.88 & 12.7 & $2.57 \pm 0.22$ & 8.4 & Lie16 \\
\hline XRF $121229 \mathrm{~A}$ & 2.707 & 111.46 & $-2.27 \pm 0.19$ & $-1.32_{-0.38}^{+1.19}$ & $77.5 \pm 10.6$ & $1.49 \pm 0.40$ & 27.6 & Lie16 \\
\hline XRF $130608 \mathrm{~A}$ & - & 151.85 & $-2.62 \pm 0.20$ & -1.93 & $87.8 \pm 8.4$ & $1.53 \pm 0.36$ & 3.4 & Lie16 \\
\hline XRF $130612 \mathrm{~A}$ & 2.006 & 4.00 & $-1.99 \pm 0.12$ & $0.84_{-0.75}^{+1.11}$ & $23.0 \pm 2.1$ & $1.96 \pm 0.51$ & $37.3_{-2.6}^{+3.4}$ & Lie16 \\
\hline XRF 130615A & - & 332.56 & $-2.09 \pm 0.08$ & $-0.72_{-0.41}^{+0.52}$ & $199.7 \pm 12.8$ & $1.46 \pm 0.20$ & $35.5_{-3.6}^{+3.0}$ & Lie16 \\
\hline XRF $131002 B$ & - & 52.00 & $-2.23 \pm 0.08$ & $\begin{array}{r}-0.89_{-0.38}^{+0.41} \\
+0.46\end{array}$ & $68.9 \pm 3.9$ & $1.62 \pm 0.22$ & $31.0_{-5.1}^{+2.6}$ & Lie16 \\
\hline XRF $131018 \mathrm{~A}$ & - & 73.22 & $-2.24 \pm 0.07$ & $\begin{array}{r}-1.71_{-0.14}^{+0.38} \\
\end{array}$ & $102.5 \pm 5.4$ & $1.34 \pm 0.13$ & $20.8_{-9.8}^{+6.1}$ & Lie16 \\
\hline XRF 131120A & - & 130.43 & $-2.87 \pm 0.24$ & -0.79 & $61.9 \pm 7.5$ & $1.89 \pm 0.56$ & 17.1 & Lie16 \\
\hline XRF $131127 \mathrm{~A}$ & - & 94.97 & $-2.35 \pm 0.08$ & $\begin{array}{l}-1.82_{-0.09}^{+0.35} \\
\end{array}$ & $187.6 \pm 10.1$ & $1.44 \pm 0.16$ & $12.8_{-6.0}^{+8.1}$ & Lie16 \\
\hline XRF $140622 \mathrm{~A}$ & 0.959 & 0.13 & $-3.08 \pm 0.15$ & $\begin{array}{l}-0.09 \\
1.23_{-0.65}^{+1.17}\end{array}$ & $2.7 \pm 0.3$ & $1.39 \pm 0.30$ & $44.2_{-3.9}^{+6.0}$ & Lie16 \\
\hline XRF $140730 \mathrm{~A}$ & - & 40.90 & $-2.39 \pm 0.15$ & $-1.68_{-0.16}^{+0.81}$ & $27.8 \pm 3.0$ & $1.60 \pm 0.35$ & 16.2 & Lie16 \\
\hline XRF 141026A & - & 139.48 & $-2.33 \pm 0.09$ & -1.99 & $123.9 \pm 7.3$ & $1.43 \pm 0.18$ & $\mathbf{0 . 9 _ { - 0 . 2 } ^ { + 0 . 7 }}$ & Lie16 \\
\hline XRF 150120B & - & 24.34 & $-2.24 \pm 0.13$ & -2.22 & $50.1 \pm 4.7$ & $1.36 \pm 0.22$ & - & - \\
\hline XRF $150201 \mathrm{~A}$ & - & 26.59 & $-2.35 \pm 0.13$ & $-0.98_{-0.45}^{+0.84}$ & $69.7 \pm 6.3$ & $1.80 \pm 0.42$ & $27.3_{-13.5}^{+4.4}$ & Lie16 \\
\hline XRF $150720 \mathrm{~A}$ & - & 238.54 & $-2.48 \pm 0.19$ & $\begin{array}{r}-1.98_{-0.01}^{+0.45} \\
-0.83\end{array}$ & $70.6 \pm 13.4$ & $1.47 \pm 0.57$ & $1.2_{-0.4}^{+13.0}$ & Lie16 \\
\hline XRF $150821 \mathrm{~A}$ & 0.755 & 168.94 & $-1.93 \pm 0.17$ & $0.61_{-0.93}^{+1.49}$ & $71.3 \pm 9.2$ & $1.45 \pm 0.38$ & $\begin{array}{l}{ }^{-0}+1_{-4.5}^{+7.1} \\
42.1_{-4}\end{array}$ & Lie16 \\
\hline XRF 150901A & - & 64.00 & $-2.59 \pm 0.25$ & -1.87 & $45.6 \pm 6.6$ & $1.57 \pm 0.45$ & 5.8 & Lie16 \\
\hline XRF 150915A & 1.968 & 160.00 & $-2.47 \pm 0.21$ & -1.92 & $71.9 \pm 13.4$ & $1.39 \pm 0.51$ & 4.9 & Lie16 \\
\hline XRF 151023A & - & 10.76 & $-2.25 \pm 0.12$ & $-1.36_{-0.39}^{+.64}$ & $24.3 \pm 2.0$ & $1.46 \pm 0.23$ & 27.3 & Lie16 \\
\hline XRF $151029 \mathrm{~A}$ & 1.423 & 8.95 & $-2.18 \pm 0.10$ & $\begin{array}{r}-0.39 \\
-0.28_{-0.51}^{+0.65}\end{array}$ & $38.0 \pm 2.8$ & $1.76 \pm 0.33$ & $34.0_{-34}^{+3.0}$ & Lie16 \\
\hline XRF 160117B & 0.87 & 11.54 & $-2.29 \pm 0.11$ & $-1.78_{-.11}^{+0.55}$ & $31.7 \pm 2.5$ & $1.40 \pm 0.22$ & $16.1_{-7.8}^{+11.8}$ & Lie16 \\
\hline XRF $160501 \mathrm{~A}$ & - & - & $-2.28 \pm 0.12$ & $-0.64_{-0.61}^{+\dot{0} 0.79}$ & $53.8 \pm 4.5$ & $1.77 \pm 0.36$ & 31.3 & Lie16 \\
\hline XRF $160525 \mathrm{~A}$ & - & - & $-2.62 \pm 0.27$ & $0.93_{-1.29}^{+3.61}$ & $16.7 \pm 2.4$ & $6.50 \pm 5.47$ & $25.3_{-38}^{+3.5}$ & Lie16 \\
\hline XRF 160827A & - & 14.05 & $-2.14 \pm 0.15$ & $0.24_{-.87}^{+1.29}$ & $33.0 \pm 3.5$ & $1.88 \pm 0.52$ & $35.6_{-5.5}^{+4.2}$ & Lie16 \\
\hline XRR 041219B & - & 9.86 & $-2.03 \pm 0.16$ & $-2.17_{-0.17}^{+0.51}$ & 26.2 & $1.05 \pm 0.26$ & 72.2 & Lie16 \\
\hline XRR 041220 & - & 5.58 & $-1.67 \pm 0.06$ & $\begin{array}{r}-0.17 \\
-1.53_{-0.13}^{+0.25}\end{array}$ & $36.1 \pm 1.6$ & $0.80 \pm 0.05$ & $155.3 \pm 43.7$ & Lie16 \\
\hline XRR 041224 & - & 177.56 & $-1.73 \pm 0.03$ & $-0.98 \pm 0.13$ & $855.1 \pm 17.2$ & $0.84 \pm 0.03$ & $68.9_{-3.4}^{+5.8}$ & Lie16 \\
\hline XRR 041228 & - & 56.30 & $-1.59 \pm 0.04$ & $-1.59_{-0.04}^{+0.07}$ & $357.4 \pm 10.6$ & $0.75 \pm 0.04$ & -3.4 & - \\
\hline XRR 050223 & 0.584 & 22.68 & $-1.82 \pm 0.08$ & $-1.46 \pm 0.33$ & $62.7 \pm 3.8$ & $0.92 \pm 0.08$ & 68.1 & Lie16 \\
\hline XRR 050315 & 1.95 & 95.40 & $-2.09 \pm 0.04$ & $-1.77_{-0.09}^{+0.18}$ & $307.9 \pm 8.4$ & $1.09 \pm 0.05$ & $33.9_{-14.3}^{+6.6}$ & Lie16 \\
\hline XRR 050318 & 1.4436 & 16.12 & $-1.89 \pm 0.05$ & $\begin{array}{r}-0.09 \\
-1.03 \pm 0.20\end{array}$ & $104.4 \pm 3.7$ & $1.04 \pm 0.06$ & $49.2_{-3.0}^{+4.7}$ & Lie16 \\
\hline XRR 050319 & 3.2425 & 151.58 & $-1.93 \pm 0.09$ & $-1.62_{-0.28}^{+0.37}$ & $129.0 \pm 8.6$ & $1.00 \pm 0.10$ & $51.4 \pm 24.6$ & Lie16 \\
\hline XRR 050410 & - & 64.00 & $-1.62 \pm 0.04$ & $-0.81 \pm 0.18$ & $434.9 \pm 12.4$ & $0.78 \pm 0.03$ & $75.6_{-5.1}^{+10.5}$ & Lie16 \\
\hline
\end{tabular}


Table 2-Continued

\begin{tabular}{|c|c|c|c|c|c|c|c|c|}
\hline GRB Name & $\mathrm{z}$ & $\begin{array}{c}T_{90} \\
(\mathrm{~s})\end{array}$ & $\alpha_{P L}$ & $\alpha_{C P L}$ & $\begin{array}{c}S(15-150 \mathrm{keV}) \\
\left(10^{-8} \mathrm{erg} \mathrm{cm}^{-2}\right)\end{array}$ & SR & $\begin{array}{l}E_{\text {peak }}^{o b s} \\
(\mathrm{keV})\end{array}$ & Ref \\
\hline XRR 050418 & - & 81.76 & $-1.67 \pm 0.03$ & $-1.28 \pm 0.12$ & $510.2 \pm 10.0$ & $0.79 \pm 0.02$ & $98.2_{-10.1}^{+38.5}$ & Lie16 \\
\hline XRR 050421 & - & 8.41 & $-1.67 \pm 0.22$ & $-0.43_{-0.85}^{+1.53}$ & $8.4 \pm 1.4$ & $0.90 \pm 0.25$ & $\begin{array}{l}-10.1 \\
58.1 \pm 22.4\end{array}$ & Lie16 \\
\hline XRR 050502B & 5.2 & 17.72 & $-1.58 \pm 0.07$ & $-1.15_{-0.24}^{+0.31}$ & $45.1 \pm 2.5$ & $0.78 \pm 0.06$ & $89.8 \pm 15.0$ & Lie16 \\
\hline XRR 050507 & - & 15.00 & $-1.60 \pm 0.10$ & $0.53_{-0.53}^{+0.69}$ & $27.3 \pm 2.3$ & $0.90 \pm 0.25$ & $55.5_{-4.1}^{+6.8}$ & Lie16 \\
\hline XRR 050509A & - & 10.51 & $-2.09 \pm 0.08$ & $-2.09 \pm 0.08$ & 33.5 & $1.23 \pm 0.11$ & - & - \\
\hline XRR 050509B & 0.2249 & 0.02 & $-1.28 \pm 0.20$ & $2.19_{-1.36}^{+3.37}$ & $0.6 \pm 0.1$ & $0.75 \pm 0.18$ & $58.9_{-5.3}^{+20.0}$ & Lie16 \\
\hline XRR $050525 \mathrm{~A}$ & 0.606 & 8.84 & -1.78 & $-1.03 \pm 0.05$ & $1513.1 \pm 10.0$ & $0.79 \pm 0.01$ & $80.4_{-1.5}^{+1.7}$ & Lie16 \\
\hline XRR 050607 & - & 48.00 & $-1.92 \pm 0.09$ & $-1.80_{-0.24}^{+0.42}$ & $60.3 \pm 3.9$ & $0.96 \pm 0.10$ & $73.4 \pm 36.7$ & Lie16 \\
\hline XRR 050701 & - & 21.70 & $-1.68 \pm 0.04$ & $\begin{array}{l}-1.57_{-0.09}^{+0.17} \\
-x^{+0}\end{array}$ & $134.7 \pm 3.9$ & $0.81 \pm 0.03$ & 186.2 & Lie16 \\
\hline XRR 050713A & - & 124.67 & $-1.53 \pm 0.04$ & $\begin{array}{l}-1.41_{-0.08}^{+0.09} \\
\end{array}$ & $508.2 \pm 11.7$ & $0.72 \pm 0.03$ & 252.6 & Lie16 \\
\hline XRR 050715 & - & 187.00 & $-1.66 \pm 0.07$ & $-1.66_{-0.29}^{+0.08}$ & $178.3 \pm 8.0$ & 0.78 & - & - \\
\hline XRR 050721 & - & 104.70 & $-1.86 \pm 0.09$ & $\begin{array}{r}-1.09_{-0.39}^{+0.49} \\
-{ }_{0}\end{array}$ & $373.8 \pm 24.9$ & $0.97 \pm 0.10$ & $54.4_{-6.6}^{+258.2}$ & Lie16 \\
\hline XRR 050724 & 0.257 & 98.68 & $-1.93 \pm 0.10$ & $\begin{array}{r}-2.02_{-0.14}^{+0.29} \\
\end{array}$ & $89.0 \pm 14.8$ & $0.94 \pm 0.15$ & 11.5 & Lie16 \\
\hline XRR 050801 & 1.38 & 19.57 & $-2.00 \pm 0.12$ & $\begin{array}{l}-2.03_{-0.05}^{+0.156} \\
\end{array}$ & $31.1 \pm 2.5$ & $0.99 \pm 0.13$ & 36.1 & Lie16 \\
\hline XRR 050814 & 5.3 & 142.85 & $-1.75 \pm 0.08$ & $\begin{array}{r}-0.99_{-0.34}^{+0.40} \\
-\end{array}$ & $179.2 \pm 11.4$ & $0.90 \pm 0.09$ & $61.8_{-6.9}^{+55.2}$ & Lie16 \\
\hline XRR 050906 & - & - & $-1.66 \pm 0.26$ & $\begin{array}{l}2.75_{-1.32}^{+3.62} \\
x_{-1.34}\end{array}$ & $0.6 \pm 0.1$ & $0.78 \pm 0.16$ & $\begin{array}{r}57.0_{-7.9}^{+8.3} \\
\end{array}$ & Lie16 \\
\hline XRR 050908 & 3.3467 & 18.28 & $-1.83 \pm 0.08$ & $-0.94_{-0.34}^{+0.41}$ & $44.2 \pm 2.9$ & $1.02 \pm 0.11$ & $50.5_{-4.8}^{+15.7}$ & Lie16 \\
\hline XRR 050911 & - & 16.24 & $-1.82 \pm 0.14$ & $\begin{array}{l}-1.79_{-0.48}^{+0.54} \\
-{ }_{-0.57}\end{array}$ & $31.9 \pm 3.2$ & $0.88 \pm 0.13$ & $\begin{array}{l}-4.0 \\
-\end{array}$ & - \\
\hline XRR 050915B & - & 43.10 & $-1.89 \pm 0.03$ & $\begin{array}{r}-0.48 \\
-1.37 \pm 0.13\end{array}$ & $340.4 \pm 7.1$ & $0.95 \pm 0.03$ & $59.4_{-3.6}^{+7.3}$ & Lie16 \\
\hline XRR 050916 & - & 85.18 & $-1.79 \pm 0.11$ & $\begin{array}{l}-0.93_{-0.49}^{+0.75} \\
\text { (1) }\end{array}$ & $104.6 \pm 9.7$ & $0.97 \pm 0.13$ & $54.7 \pm 7.1$ & Lie16 \\
\hline XRR 050922B & 4.5 & 157.02 & $-2.11 \pm 0.13$ & $-1.82_{-0.31}^{+0.56}$ & $229.7 \pm 21.2$ & $1.14 \pm 0.18$ & $25.0 \pm 11.8$ & Lie16 \\
\hline XRR 051001 & 2.4296 & 190.26 & $-2.02 \pm 0.07$ & $-1.63 \pm 0.26$ & $168.8 \pm 8.6$ & $1.08 \pm 0.09$ & $39.9 \pm 15.7$ & Lie16 \\
\hline XRR 051012 & - & 12.80 & $-2.11 \pm 0.10$ & $-1.31_{-0.43}^{+0.55}$ & $25.0 \pm 1.9$ & $1.27 \pm 0.18$ & $34.2_{-17.0}^{+7.7}$ & Lie16 \\
\hline XRR 051016A & - & 21.00 & $-1.74 \pm 0.12$ & $\begin{array}{r}-1.47_{-0.35}^{+0.58} \\
-\end{array}$ & $82.7 \pm 7.0$ & $0.85 \pm 0.12$ & 95.6 & Lie16 \\
\hline XRR 051021B & - & 46.51 & $-1.54 \pm 0.07$ & $\begin{array}{r}-0.48_{-0.31}^{+0.36} \\
-0.36\end{array}$ & $81.9 \pm 4.5$ & $0.78 \pm 0.06$ & $68.7_{-5.9}^{+15.9}$ & Lie16 \\
\hline XRR 051113 & - & 93.30 & $-1.69 \pm 0.07$ & $\begin{array}{l}-1.49_{-0.13}^{+0.27} \\
\end{array}$ & $255.1 \pm 11.5$ & $0.81 \pm 0.06$ & $130.0 \pm 31.5$ & Lie16 \\
\hline XRR 051117A & - & 140.63 & $-1.82 \pm 0.03$ & $-1.53 \pm 0.13$ & $429.9 \pm 9.5$ & $0.89 \pm 0.03$ & $80.4_{-9.2}^{+112.5}$ & Lie16 \\
\hline XRR 051117B & - & 9.02 & $-1.54 \pm 0.15$ & $-0.54_{-0.56}^{+0.74}$ & $16.4 \pm 1.9$ & $0.77 \pm 0.13$ & $70.8 \pm 10.6$ & Lie16 \\
\hline XRR 051213 & - & 71.06 & $-1.60 \pm 0.10$ & $\begin{array}{r}-0.74_{-0.40}^{+0.56} \\
-0.50\end{array}$ & $73.6 \pm 5.8$ & $0.83 \pm 0.10$ & $66.6_{-8.4}^{+103.7}$ & Lie16 \\
\hline XRR 060108 & 2.03 & 14.22 & $-2.0 \pm 0.08$ & $-2.01_{-0.05}^{+0.40}$ & $37.2 \pm 2.0$ & $1.00 \pm 0.09$ & 30.4 & Lie16 \\
\hline XRR 060109 & - & 115.59 & $-1.89 \pm 0.12$ & $\begin{array}{r}-1.31_{-0.43}^{+0.55} \\
-0.05\end{array}$ & $62.1 \pm 5.8$ & $1.02 \pm 0.15$ & $50.2 \pm 20.2$ & Lie16 \\
\hline XRR 060110 & - & 21.34 & $-1.63 \pm 0.04$ & $\begin{array}{r}-1.59_{-0.06}^{+0.43} \\
+0.12\end{array}$ & $150.0 \pm 3.3$ & $0.78 \pm 0.03$ & - & - \\
\hline XRR 060111A & - & 13.21 & $-1.62 \pm 0.03$ & $-0.88 \pm 0.14$ & $119.7 \pm 2.9$ & $0.80 \pm 0.03$ & $73.9_{-4.6}^{+8.5}$ & Lie16 \\
\hline XRR 060115 & 3.5328 & 139.09 & $-1.70 \pm 0.06$ & $-1.01 \pm 0.24$ & $171.1 \pm 7.5$ & $0.87 \pm 0.05$ & $66.1_{-6.1}^{+21.2}$ & Lie16 \\
\hline XRR 060124 & 2.3 & 13.42 & $-1.81 \pm 0.09$ & $\begin{array}{l}-1.74_{-0.32}^{+0.39} \\
\text { - }\end{array}$ & $46.1 \pm 3.0$ & $0.89 \pm 0.09$ & 148.5 & Lie16 \\
\hline XRR 060202 & 0.783 & 192.88 & $-1.77 \pm 0.06$ & $-1.77_{-0.06}^{+0.11}$ & $224.7 \pm 8.4$ & $0.85 \pm 0.02$ & - & - \\
\hline XRR 060203 & - & 69.52 & $-1.60 \pm 0.11$ & $\begin{array}{r}-1.47_{-0.18}^{+0.05} \\
-0.05\end{array}$ & $90.5 \pm 6.9$ & $0.76 \pm 0.09$ & 198.8 & Lie16 \\
\hline XRR 060206 & 4.0559 & 7.55 & $-1.66 \pm 0.04$ & $-1.12 \pm 0.15$ & $84.2 \pm 2.2$ & $0.81 \pm 0.03$ & $80.7_{-7.0}^{+19.4}$ & Lie16 \\
\hline XRR 060211A & - & 126.46 & $-1.75 \pm 0.06$ & $-0.94 \pm 0.23$ & $157.6 \pm 7.0$ & $0.92 \pm 0.06$ & $58.7_{-4.6}^{+11.1}$ & Lie16 \\
\hline XRR 060211B & - & 27.73 & $-1.53 \pm 0.10$ & $-1.19_{-0.25}^{+0.42}$ & $43.2 \pm 3.3$ & $0.74 \pm 0.08$ & 117.0 & Lie16 \\
\hline XRR 060223A & 4.41 & 11.32 & $-1.69 \pm 0.06$ & $-1.05 \pm 0.23$ & $63.9 \pm 2.7$ & $0.85 \pm 0.05$ & $69.9_{-7.0}^{+30.2}$ & Lie16 \\
\hline XRR 060223B & - & 10.36 & $-1.47 \pm 0.03$ & $-1.44 \pm 0.05$ & $84.3 \pm 4.3$ & $1.02 \pm 0.16$ & $\begin{array}{l}-1.0 \\
-\end{array}$ & - \\
\hline XRR 060306 & 1.559 & 60.94 & $-1.76 \pm 0.05$ & $-1.25 \pm 0.19$ & $205.4 \pm 7.0$ & $0.88 \pm 0.04$ & $69.4_{-6.8}^{+34.5}$ & Lie16 \\
\hline XRR 060312 & - & 44.66 & $-1.82 \pm 0.04$ & $-1.35 \pm 0.16$ & $183.7 \pm 5.0$ & $0.92 \pm 0.04$ & $64.7_{-5.4}^{+19.1}$ & Lie16 \\
\hline XRR 060322 & - & 229.47 & $-1.56 \pm 0.04$ & $-1.07 \pm 0.15$ & $521.5 \pm 13.3$ & $0.75 \pm 0.03$ & $96.8_{-10.0}^{+34.3}$ & Lie16 \\
\hline
\end{tabular}


Table 2-Continued

\begin{tabular}{|c|c|c|c|c|c|c|c|c|}
\hline GRB Name & $\mathrm{z}$ & $\begin{array}{l}T_{90} \\
(\mathrm{~s})\end{array}$ & $\alpha_{P L}$ & $\alpha_{C P L}$ & $\begin{array}{c}S(15-150 \mathrm{keV}) \\
\left(10^{-8} \mathrm{erg} \mathrm{cm}^{-2}\right)\end{array}$ & $\mathrm{SR}$ & $\begin{array}{l}E_{\text {peak }}^{o b s} \\
(\mathrm{keV})\end{array}$ & Ref \\
\hline XRR 060323 & - & 23.87 & $-1.54 \pm 0.08$ & $-1.43_{-0.42}^{+0.28}$ & $63.2 \pm 3.6$ & $0.73 \pm 0.06$ & 265.8 & Lie16 \\
\hline XRR 060413 & - & 141.12 & $-1.66 \pm 0.04$ & $-1.53 \pm 0.11$ & $351.5 \pm 8.3$ & $0.79 \pm 0.03$ & $179.3 \pm 45.0$ & Lie16 \\
\hline XRR 060418 & 1.49 & 109.08 & $-1.68 \pm 0.03$ & $-1.55 \pm 0.08$ & $831.1 \pm 14.6$ & $0.80 \pm 0.02$ & $182.8 \pm 56.3$ & Lie16 \\
\hline XRR 060421 & - & 12.00 & $-1.52 \pm 0.04$ & $-1.19 \pm 0.14$ & $122.5 \pm 3.1$ & $0.72 \pm 0.03$ & $126.3_{-18.6}^{+174.4}$ & Lie16 \\
\hline XRR 060424 & - & 37.68 & $-1.70 \pm 0.09$ & $-1.19_{-0.31}^{+0.41}$ & $65.6 \pm 4.6$ & $0.85 \pm 0.09$ & $73.4 \pm 11.6$ & Lie16 \\
\hline XRR 060427 & - & 62.00 & $-1.88 \pm 0.14$ & $-1.89 \pm 0.30$ & $47.1 \pm 5.1$ & $0.96 \pm 0.15$ & - & - \\
\hline XRR 060428A & - & 39.48 & $-2.02 \pm 0.05$ & $-1.71 \pm 0.19$ & $135.7 \pm 4.7$ & $1.05 \pm 0.06$ & $42.0 \pm 20.9$ & Lie16 \\
\hline XRR 060507 & - & 183.31 & $-1.81 \pm 0.04$ & $-1.45 \pm 0.17$ & $398.5 \pm 13.4$ & $0.96 \pm 0.05$ & $72.4_{-8.2}^{+108.1}$ & Lie16 \\
\hline XRR 060510B & 4.941 & 262.94 & $-1.77 \pm 0.04$ & $-1.59 \pm 0.12$ & $391.3 \pm 9.8$ & $0.86 \pm 0.03$ & $113.8 \pm 21.9$ & Lie16 \\
\hline XRR 060522 & 5.11 & 69.12 & $-1.54 \pm 0.07$ & $-0.70_{-0.34}^{+0.42}$ & $104.3 \pm 6.5$ & $0.78 \pm 0.07$ & $73.3_{-8.4}^{+55.4}$ & Lie16 \\
\hline XRR 060526 & 3.2213 & 298.04 & $-1.96 \pm 0.11$ & $-1.97 \pm 0.51$ & $122.4 \pm 10.0$ & $0.97 \pm 0.11$ & $24.9 \pm 5.0$ & Ama08 \\
\hline XRR 060604 & 2.1357 & 96.00 & $-2.05 \pm 0.21$ & $-2.12_{-0.07}^{+0.87}$ & $37.9 \pm 6.1$ & 1.03 & 74.5 & Lie16 \\
\hline XRR 060605 & 3.773 & 79.84 & $-1.52 \pm 0.10$ & $-0.88_{-0.38}^{+0.48}$ & $66.3 \pm 5.4$ & $0.77 \pm 0.08$ & $79.5 \pm 12.6$ & Lie16 \\
\hline XRR 060607B & - & 31.58 & $-1.62 \pm 0.06$ & $-1.52_{-0.10}^{+0.28}$ & $103.5 \pm 7.8$ & $0.97 \pm 0.10$ & 229.6 & Lie16 \\
\hline XRR 060614 & 0.1254 & 109.10 & $-2.04 \pm 0.02$ & $-2.23_{-0.11}^{+0.01}$ & $1882.9 \pm 69.7$ & $1.15 \pm 0.08$ & $98.5_{-1.8}^{+29.9}$ & Lie16 \\
\hline XRR 060707 & 3.424 & 66.64 & $-1.67 \pm 0.06$ & $-0.42_{-0.29}^{+0.34}$ & $157.7 \pm 7.5$ & $0.85 \pm 0.06$ & $61.9_{-4.1}^{+7.5}$ & Lie16 \\
\hline XRR 060708 & 1.92 & 10.03 & $-1.64 \pm 0.06$ & $\begin{array}{r}-1.22 \pm 0.22 \\
-1.29\end{array}$ & $48.0 \pm 2.1$ & $0.80 \pm 0.05$ & $88.9 \pm 12.9$ & Lie16 \\
\hline XRR 060714 & 2.7108 & 116.06 & $-1.89 \pm 0.05$ & $-1.54 \pm 0.19$ & $276.9 \pm 10.3$ & $0.97 \pm 0.05$ & $56.9 \pm 6.7$ & Lie16 \\
\hline XRR 060717 & - & 3.00 & $-1.66 \pm 0.18$ & $-0.41_{-0.85}^{+1.38}$ & $5.8 \pm 0.9$ & $0.96 \pm 0.24$ & $53.9 \pm 6.2$ & Lie16 \\
\hline XRR 060719 & 1.532 & 66.92 & $-1.89 \pm 0.05$ & $-1.67 \pm 0.18$ & $150.1 \pm 5.3$ & $0.95 \pm 0.05$ & $68.6 \pm 11.2$ & Lie16 \\
\hline XRR 060728 & - & - & $-1.44 \pm 0.22$ & $-0.17_{-0.73}^{+1.35}$ & $19.6 \pm 3.7$ & $0.79 \pm 0.59$ & $64.6 \pm 12.8$ & Lie16 \\
\hline XRR 060729 & 0.5428 & 113.04 & $-1.69 \pm 0.06$ & $-1.60 \pm 0.25$ & $258.5 \pm 12.3$ & $0.81 \pm 0.06$ & $201.2 \pm 68.9$ & Lie16 \\
\hline XRR 060804 & - & 18.19 & $-1.78 \pm 0.13$ & $-0.16_{-0.93}^{+1.68}$ & $43.7 \pm 6.4$ & $1.27 \pm 0.36$ & $43.6 \pm 4.7$ & Lie16 \\
\hline XRR 060807 & - & 54.05 & $-1.54 \pm 0.10$ & $-1.40_{-0.17}^{+0.44}$ & $85.3 \pm 6.2$ & $0.73 \pm 0.08$ & 208.7 & Lie16 \\
\hline XRR 060825 & - & 7.98 & $-1.69 \pm 0.03$ & $-1.07 \pm 0.14$ & $95.5 \pm 2.3$ & $0.84 \pm 0.03$ & $71.4_{-4.9}^{+10.4}$ & Lie16 \\
\hline XRR 060904A & 2.55 & 80.06 & $-1.54 \pm 0.02$ & $-1.51_{-0.02}^{+0.06}$ & $782.8 \pm 8.1$ & $0.73 \pm 0.01$ & $163.0 \pm 31.0$ & GCN5518 \\
\hline XRR 060904B & 0.7029 & 189.98 & $-1.66 \pm 0.07$ & $-1.23_{-0.24}^{+0.32}$ & $164.3 \pm 8.8$ & $0.82 \pm 0.06$ & $84.1 \pm 13.3$ & Lie16 \\
\hline XRR 060906 & 3.6856 & 44.59 & $-1.98 \pm 0.05$ & $-1.55 \pm 0.20$ & $220.4 \pm 7.9$ & $1.03 \pm 0.06$ & $47.8_{-9.5}^{+21.4}$ & Lie16 \\
\hline XRR 060912A & 0.937 & 5.03 & $-1.73 \pm 0.04$ & $-1.73 \pm 0.04$ & $136.4 \pm 3.0$ & $0.83 \pm 0.02$ & - & - \\
\hline XRR 060919 & - & 9.00 & $-1.83 \pm 0.09$ & $-0.84_{-0.42}^{+0.52}$ & $49.9 \pm 3.5$ & $0.98 \pm 0.11$ & $53.3_{-5.4}^{+24.4}$ & Lie16 \\
\hline XRR 060923A & 2.47 & 58.49 & $-1.78 \pm 0.11$ & $-0.28_{-0.54}^{+0.42}$ & $87.3 \pm 7.7$ & $1.02 \pm 0.15$ & $50.8_{-4.6}^{+11.5}$ & Lie16 \\
\hline XRR 060927 & 5.4636 & 22.42 & $-1.61 \pm 0.04$ & $-0.81 \pm 0.17$ & $112.0 \pm 3.4$ & $0.81 \pm 0.03$ & $70.7_{-4.8}^{+9.7}$ & Lie16 \\
\hline XRR 060929 & - & 552.96 & $-1.91 \pm 0.21$ & $-1.92 \pm 0.34$ & $73.8 \pm 20.2$ & $1.02 \pm 1.05$ & - & - \\
\hline XRR 061002 & - & 17.63 & $-1.72 \pm 0.10$ & $-1.72 \pm 0.10$ & $69.6 \pm 4.3$ & $0.82 \pm 0.10$ & - & - \\
\hline XRR 061004 & - & 6.26 & $-1.77 \pm 0.04$ & $-1.40 \pm 0.18$ & $54.8 \pm 1.8$ & $0.88 \pm 0.04$ & $74.2_{-9.0}^{+244.8}$ & Lie16 \\
\hline XRR 061006 & 0.4377 & 129.79 & $-1.74 \pm 0.08$ & $-1.74 \pm 0.08$ & $143.2 \pm 6.9$ & $0.83 \pm 0.07$ & - & - \\
\hline XRR 061019 & - & 180.38 & $-1.98 \pm 0.18$ & $-1.58_{-0.29}^{+0.76}$ & $226.9 \pm 28.6$ & $1.05 \pm 0.24$ & 44.6 & Lie16 \\
\hline XRR 061110A & 0.7578 & 44.51 & $-1.64 \pm 0.06$ & $\begin{array}{r}-1.56_{-0.10}^{+0.22} \\
+r^{+0.29}\end{array}$ & $111.6 \pm 4.6$ & $0.79 \pm 0.05$ & 240.3 & Lie16 \\
\hline XRR 061202 & - & 94.19 & $-1.55 \pm 0.03$ & $-1.44_{-0.07}^{+0.13}$ & $342.1 \pm 7.5$ & $0.73 \pm 0.02$ & 276.7 & Lie16 \\
\hline XRR 061222B & 3.355 & 37.25 & $-1.96 \pm 0.06$ & $-1.31 \pm 0.25$ & $215.4 \pm 10.1$ & $1.05 \pm 0.24$ & $47.3_{-5.7}^{+10.3}$ & Lie16 \\
\hline XRR 070103 & 2.6208 & 18.41 & $-1.89 \pm 0.10$ & $-1.22_{-0.41}^{+0.53}$ & $30.3 \pm 2.7$ & $1.06 \pm 0.15$ & $46.6 \pm 20.8$ & Lie16 \\
\hline XRR 070110 & 2.3521 & 88.43 & $-1.57 \pm 0.06$ & $-1.57 \pm 0.06$ & $164.7 \pm 5.4$ & $0.74 \pm 0.03$ & $110.4 \pm 50.7$ & Lia15 \\
\hline XRR 070129 & 2.3384 & 459.75 & $-1.99 \pm 0.07$ & $-1.63_{-0.26}^{+0.35}$ & $288.0 \pm 16.7$ & $1.05 \pm 0.10$ & $43.5 \pm 13.2$ & Lie16 \\
\hline XRR 070208 & 1.165 & 64.00 & $-1.97 \pm 0.18$ & $\begin{array}{r}-1.65_{-0.26}^{+0.20} \\
+0.00\end{array}$ & $50.8 \pm 6.2$ & $1.00 \pm 0.23$ & $51.3 \pm 25.0$ & Lie16 \\
\hline XRR 070219 & - & 18.00 & $-1.81 \pm 0.11$ & $-1.81_{-0.16}^{+0.32}$ & $28.1 \pm 2.6$ & $0.86 \pm 0.06$ & - & - \\
\hline
\end{tabular}


Table 2-Continued

\begin{tabular}{|c|c|c|c|c|c|c|c|c|}
\hline GRB Name & $\mathrm{z}$ & $\begin{array}{c}T_{90} \\
(\mathrm{~s})\end{array}$ & $\alpha_{P L}$ & $\alpha_{C P L}$ & $\begin{array}{c}S(15-150 \mathrm{keV}) \\
\left(10^{-8} \mathrm{erg} \mathrm{cm}^{-2}\right)\end{array}$ & $\mathrm{SR}$ & $\begin{array}{l}E_{\text {peak }}^{o b s} \\
(\mathrm{keV})\end{array}$ & Ref \\
\hline XRR 070223 & - & 128.00 & $-1.89 \pm 0.06$ & $-1.48 \pm 0.23$ & $169.7 \pm 8.3$ & $0.98 \pm 0.07$ & $54.4 \pm 7.2$ & Lie16 \\
\hline XRR 070306 & 1.49594 & 209.24 & $-1.63 \pm 0.04$ & $-1.60_{-0.06}^{+0.12}$ & $545.6 \pm 13.5$ & 0.77 & - & - \\
\hline XRR 070411 & 2.9538 & 115.69 & $-1.66 \pm 0.05$ & $-1.66 \pm 0.05$ & $272.4 \pm 7.5$ & $0.79 \pm 0.03$ & - & - \\
\hline XRR 070412 & - & 33.88 & $-1.41 \pm 0.10$ & $-0.34_{-0.44}^{+0.54}$ & $42.4 \pm 3.8$ & $0.75 \pm 0.09$ & $69.9_{-8.3}^{+51.7}$ & Lie16 \\
\hline XRR 070419B & 1.9588 & 238.01 & $-1.65 \pm 0.03$ & $\begin{array}{r}-1.62_{-0.04}^{+0.08} \\
+0.04\end{array}$ & $744.5 \pm 10.1$ & $0.79 \pm 0.02$ & $27.0_{-9.0}^{+8.0}$ & Lia15 \\
\hline XRR 070429A & - & 168.00 & $-2.10 \pm 0.13$ & $\begin{array}{r}-2.18_{-0.28}^{+0.20} \\
+0.20\end{array}$ & $78.1 \pm 15.9$ & $1.17 \pm 0.41$ & 81.8 & Lie16 \\
\hline XRR 070429B & 0.9023 & 0.49 & $-1.65 \pm 0.11$ & $-1.10_{-0.36}^{+0.28}$ & $6.2 \pm 0.6$ & $0.84 \pm 0.12$ & $72.9 \pm 13.0$ & Lie16 \\
\hline XRR 070506 & 2.309 & 5.99 & $-1.73 \pm 0.09$ & $\begin{array}{r}-0.77_{-0.38}^{+0.46} \\
-0.36\end{array}$ & $20.7 \pm 1.6$ & $0.95 \pm 0.11$ & $55.1_{-5.6}^{+24.6}$ & Lie16 \\
\hline XRR 070509 & - & 7.83 & $-2.21 \pm 0.11$ & $-1.95_{-0.01}^{+0.38}$ & $17.9 \pm 1.6$ & $1.27 \pm 0.19$ & $\begin{array}{l}-5.6 \\
6.6 \pm 3.1\end{array}$ & Lie16 \\
\hline XRR 070517 & - & 7.82 & $-1.91 \pm 0.12$ & $-1.18_{-0.46}^{+0.61}$ & $20.1 \pm 2.0$ & $1.07 \pm 0.18$ & 46.4 & Lie16 \\
\hline XRR 070518 & - & 5.50 & $-2.01 \pm 0.11$ & $-1.84_{-0.28}^{+0.54}$ & $16.4 \pm 1.4$ & $1.04 \pm 0.16$ & 37.5 & Lie16 \\
\hline XRR 070520A & - & 48.00 & $-1.69 \pm 0.15$ & $-0.29_{-0.61}^{+0.82}$ & $35.7 \pm 4.0$ & $0.86 \pm 0.15$ & $60.3_{-7.5}^{+34.3}$ & Lie16 \\
\hline XRR 070521 & 2.0865 & 38.63 & $-1.33 \pm 0.02$ & $-1.11 \pm 0.07$ & $331.2 \pm 10.7$ & $0.99 \pm 0.04$ & $237.7_{-36.4}^{+166.0}$ & Lie16 \\
\hline XRR 070611 & 2.0394 & 13.18 & $-1.66 \pm 0.12$ & $-0.75_{-0.51}^{+0.83}$ & $37.2 \pm 3.7$ & $0.87 \pm 0.13$ & $62.9 \pm 6.5$ & Lie16 \\
\hline XRR 070612A & 0.617 & 365.28 & $-1.64 \pm 0.05$ & $-1.44 \pm 0.12$ & $1065.9 \pm 33.4$ & $0.79 \pm 0.04$ & 137.7 & Lie16 \\
\hline XRR 070612B & - & 18.00 & $-1.56 \pm 0.06$ & $-1.08 \pm 0.23$ & $177.0 \pm 7.3$ & $0.74 \pm 0.05$ & $100.8_{-14.4}^{+411.8}$ & Lie16 \\
\hline XRR 070616 & - & 406.88 & $-1.59 \pm 0.02$ & $-1.47 \pm 0.07$ & $1949.4 \pm 15.0$ & $0.75 \pm 0.01$ & $245.0 \pm 50.6$ & Lie16 \\
\hline XRR 070621 & - & 33.26 & $-1.53 \pm 0.03$ & $-1.48 \pm 0.08$ & $440.0 \pm 7.8$ & $0.72 \pm 0.02$ & - & - \\
\hline XRR 070704 & - & 384.94 & $-1.65 \pm 0.04$ & $-1.57_{-0.07}^{+0.13}$ & $589.2 \pm 14.0$ & $0.78 \pm 0.03$ & $264.3 \pm 79.6$ & Lie16 \\
\hline XRR 070724A & 0.4571 & 0.43 & $-1.86 \pm 0.16$ & $-0.68_{-0.65}^{+0.91}$ & $3.0 \pm 0.4$ & $0.96 \pm 0.23$ & $45.9 \pm 8.6$ & Lie16 \\
\hline XRR 070731 & - & 2.86 & $-1.58 \pm 0.11$ & $-0.55_{-0.51}^{+0.63}$ & $15.3 \pm 1.4$ & $0.83 \pm 0.11$ & $64.3_{-7.9}^{+85.5}$ & Lie16 \\
\hline XRR 070802 & 2.4541 & 15.80 & $-1.75 \pm 0.13$ & $\begin{array}{r}-1.04_{-0.28}^{+0.75} \\
-0.01\end{array}$ & $25.9 \pm 2.8$ & $0.93 \pm 0.15$ & 58.3 & Lie16 \\
\hline XRR 070805 & - & 26.43 & $-1.73 \pm 0.09$ & $\begin{array}{r}-0.88 \\
-1.73 \pm 0.09\end{array}$ & $55.1 \pm 2.9$ & $0.93 \pm 0.09$ & - & - \\
\hline XRR 070809 & 0.2187 & 1.28 & $-1.66 \pm 0.11$ & $-1.43_{-0.22}^{+0.54}$ & $7.3 \pm 0.5$ & $0.95 \pm 0.14$ & $111.1 \pm 43.2$ & Lie16 \\
\hline XRR 070810A & 2.17 & 9.04 & $-2.05 \pm 0.06$ & $\begin{array}{r}-0.22 \\
-1.37 \pm 0.28\end{array}$ & $62.9 \pm 3.0$ & $1.15 \pm 0.09$ & $39.0_{-10.9}^{+5.7}$ & Lie16 \\
\hline XRR 070911 & - & 161.72 & $-1.71 \pm 0.02$ & $-1.67_{-0.03}^{+0.07}$ & $1267.7 \pm 12.7$ & $0.82 \pm 0.01$ & $481.7 \pm 161.8$ & Lie16 \\
\hline XRR 070913 & - & 2.68 & $-1.41 \pm 0.12$ & $0.72_{-0.70}^{+1.07}$ & $14.8 \pm 1.5$ & $0.75 \pm 0.11$ & $62.3_{-5.8}^{+15.3}$ & Lie16 \\
\hline XRR 070917 & - & 8.72 & $-1.50 \pm 0.03$ & $-1.41 \pm 0.06$ & $123.9 \pm 5.9$ & $0.97 \pm 0.04$ & 364.5 & Lie16 \\
\hline XRR 070920A & - & 55.76 & $-1.60 \pm 0.10$ & $-1.16_{-0.21}^{+0.48}$ & $49.2 \pm 4.2$ & $0.80 \pm 0.10$ & $85.9 \pm 20.4$ & Lie16 \\
\hline XRR 070920B & - & 22.26 & $-1.87 \pm 0.05$ & $-0.39 \pm 0.27$ & $69.5 \pm 2.8$ & $1.18 \pm 0.14$ & $44.7_{-1.9}^{+2.6}$ & Lie16 \\
\hline XRR 071001 & - & 57.43 & $-1.67 \pm 0.08$ & $-1.36_{-0.25}^{+0.18}$ & $76.0 \pm 4.6$ & $0.83 \pm 0.07$ & $92.2 \pm 18.3$ & Lie16 \\
\hline XRR 071013 & - & 75.58 & $-1.61 \pm 0.25$ & $-1.64_{-0.75}^{+0.17}$ & $25.0 \pm 5.9$ & $0.93 \pm 0.33$ & - & - \\
\hline XRR 071018 & - & 273.57 & $-1.56 \pm 0.12$ & $-1.57 \pm 0.12$ & $106.0 \pm 8.3$ & $0.73 \pm 0.07$ & - & - \\
\hline XRR 071021 & 2.452 & 228.72 & $-1.66 \pm 0.10$ & $-1.65_{-0.44}^{+0.16}$ & $137.2 \pm 7.9$ & $0.78 \pm 0.08$ & - & - \\
\hline XRR 071025 & 5.2 & 241.30 & $-1.71 \pm 0.03$ & $-1.65 \pm 0.06$ & $727.5 \pm 12.9$ & $0.82 \pm 0.02$ & 295.0 & Lie16 \\
\hline XRR 071028A & - & 26.86 & $-1.79 \pm 0.12$ & $-0.74_{-0.56}^{+0.78}$ & $26.6 \pm 2.9$ & $1.05 \pm 0.19$ & $49.0 \pm 6.2$ & Lie16 \\
\hline XRR 071118 & - & 105.60 & $-1.53 \pm 0.14$ & $-1.14_{-0.33}^{+0.66}$ & $63.3 \pm 7.4$ & $0.75 \pm 0.12$ & 99.7 & Lie16 \\
\hline XRR 071122 & 1.14 & 71.43 & $-1.62 \pm 0.15$ & $-1.42_{-0.25}^{+0.61}$ & $60.7 \pm 6.4$ & $0.78 \pm 0.13$ & 138.9 & Lie16 \\
\hline XRR 071129 & - & 408.08 & $-1.86 \pm 0.07$ & $\begin{array}{r}-0.25 \\
-1.47 \pm 0.24\end{array}$ & $351.6 \pm 18.5$ & $0.96 \pm 0.07$ & $59.3 \pm 7.2$ & Lie16 \\
\hline XRR 080121 & - & 0.32 & $-1.61 \pm 0.23$ & $-1.32_{-0.54}^{+0.89}$ & $3.2 \pm 0.5$ & $0.79 \pm 0.20$ & 107.7 & Lie16 \\
\hline XRR 080123 & 0.495 & 114.91 & $-2.07 \pm 0.25$ & -1.98 & $55.2 \pm 8.5$ & $1.06 \pm 0.28$ & 8.2 & Lie16 \\
\hline XRR 080205 & - & 106.30 & $-1.96 \pm 0.06$ & $-1.83 \pm 0.20$ & $200.4 \pm 7.2$ & $0.99 \pm 0.07$ & $58.8 \pm 24.2$ & Lie16 \\
\hline XRR 080207 & 2.0858 & 292.46 & $-1.56 \pm 0.03$ & $-1.25 \pm 0.12$ & $623.1 \pm 12.1$ & $0.74 \pm 0.04$ & $124.6_{-16.5}^{+90.2}$ & Lie16 \\
\hline XRR 080210 & 2.6419 & 42.26 & $-1.72 \pm 0.05$ & $-1.71 \pm 0.06$ & $182.4 \pm 5.7$ & 0.82 & - & - \\
\hline XRR 080212 & - & 126.31 & $-1.51 \pm 0.06$ & $-0.23 \pm 0.28$ & $301.4 \pm 12.9$ & $0.74 \pm 0.04$ & $69.9_{-4.5}^{+8.6}$ & Lie16 \\
\hline
\end{tabular}


Table 2-Continued

\begin{tabular}{|c|c|c|c|c|c|c|c|c|}
\hline GRB Name & $\mathrm{z}$ & $\begin{array}{l}T_{90} \\
(\mathrm{~s})\end{array}$ & $\alpha_{P L}$ & $\alpha_{C P L}$ & $\begin{array}{c}S(15-150 \mathrm{keV}) \\
\left(10^{-8} \mathrm{erg} \mathrm{cm}^{-2}\right)\end{array}$ & $\mathrm{SR}$ & $\begin{array}{l}E_{p e a k}^{o b s} \\
(\mathrm{keV})\end{array}$ & Ref \\
\hline XRR 080229A & - & 64.00 & $-1.81 \pm 0.03$ & $-1.81 \pm 0.03$ & $912.9 \pm 16.2$ & $0.88 \pm 0.03$ & - & - \\
\hline XRR 080307 & - & 124.00 & $-1.70 \pm 0.10$ & $-1.70_{-0.10}^{+0.27}$ & $94.4 \pm 4.5$ & $0.80 \pm 0.05$ & - & - \\
\hline XRR 080310 & 2.42743 & 363.21 & $-2.22 \pm 0.07$ & $-1.65_{-0.18}^{+0.38}$ & $225.7 \pm 9.9$ & $1.25 \pm 0.10$ & $23.3_{-11.4}^{+5.7}$ & Lie16 \\
\hline XRR 080319A & - & 43.62 & $-1.54 \pm 0.06$ & $-1.32_{-0.14}^{+0.18}$ & $440.9 \pm 16.2$ & $0.73 \pm 0.05$ & 167.7 & Lie16 \\
\hline XRR 080320 & - & 13.82 & $-1.62 \pm 0.11$ & $-1.51_{-0.16}^{+0.45}$ & $28.0 \pm 2.3$ & $0.78 \pm 0.09$ & 189.7 & Lie16 \\
\hline XRR 080325 & 1.78 & 166.74 & $-1.56 \pm 0.08$ & $-1.56_{-0.08}^{+0.17}$ & $503.8 \pm 22.4$ & $0.75 \pm 0.04$ & - & - \\
\hline XRR 080409 & - & 22.66 & $-2.04 \pm 0.09$ & $\begin{array}{r}-0.69_{-0.45}^{+0.08} \\
-0.56\end{array}$ & $56.1 \pm 3.9$ & $1.28 \pm 0.17$ & $40.3 \pm 4.5$ & Lie16 \\
\hline XRR 080411 & 1.0301 & 56.33 & $-1.68 \pm 0.01$ & $-1.66 \pm 0.02$ & $2645.4 \pm 16.1$ & $0.80 \pm 0.01$ & $258.1 \pm 34.5$ & Ama08 \\
\hline XRR 080413A & 2.433 & 46.36 & $-1.53 \pm 0.03$ & $-1.43_{-0.07}^{+0.11}$ & $349.0 \pm 5.9$ & $0.72 \pm 0.02$ & $170.1 \pm 52.4$ & Ama08 \\
\hline XRR 080426 & - & 1.73 & $-1.91 \pm 0.06$ & $-1.75 \pm 0.20$ & $36.9 \pm 1.5$ & $0.96 \pm 0.06$ & $69.8 \pm 25.5$ & Lie16 \\
\hline XRR 080430 & 0.767 & 13.87 & $-1.73 \pm 0.04$ & $-1.73 \pm 0.08$ & $118.7 \pm 2.4$ & 0.83 & - & - \\
\hline XRR 080503 & - & 176.00 & $-1.87 \pm 0.06$ & $-1.68 \pm 0.21$ & $199.2 \pm 8.7$ & $0.94 \pm 0.06$ & $73.2 \pm 28.7$ & Lie16 \\
\hline XRR 080506 & - & 152.20 & $-1.75 \pm 0.09$ & $-1.76 \pm 0.09$ & $130.5 \pm 8.0$ & $0.84 \pm 0.05$ & - & - \\
\hline XRR 080516 & 3.2 & 5.76 & $-1.82 \pm 0.13$ & $-1.56_{-0.35}^{+0.59}$ & $26.1 \pm 2.4$ & $0.91 \pm 0.13$ & $76.6 \pm 38.2$ & Lie16 \\
\hline XRR 080603B & 2.6892 & 59.12 & $-1.73 \pm 0.03$ & $\begin{array}{r}-0.35 \\
-1.21 \pm 0.13\end{array}$ & $246.4 \pm 5.3$ & $0.85 \pm 0.03$ & $74.9_{-5.4}^{+12.9}$ & Lie16 \\
\hline XRR 080604 & 1.4171 & 77.61 & $-1.68 \pm 0.09$ & $-1.19_{-0.30}^{+0.42}$ & $74.4 \pm 5.5$ & $0.86 \pm 0.09$ & $72.3 \pm 11.2$ & Lie16 \\
\hline XRR 080701 & - & 17.82 & $-2.12 \pm 0.08$ & $-1.65_{-0.19}^{+0.35}$ & $70.0 \pm 4.2$ & $1.20 \pm 0.12$ & 29.8 & Lie16 \\
\hline XRR 080703 & - & 3.52 & $-1.45 \pm 0.11$ & $-0.42_{-0.50}^{+0.19}$ & $19.0 \pm 1.9$ & $0.75 \pm 0.10$ & $71.5_{-10.2}^{+214.8}$ & Lie16 \\
\hline XRR 080707 & 1.2322 & 30.16 & $-1.70 \pm 0.08$ & $-1.70 \pm 0.09$ & $62.9 \pm 3.5$ & 0.81 & - & - \\
\hline XRR 080723A & - & 17.34 & $-1.72 \pm 0.10$ & $-1.43_{-0.24}^{+0.50}$ & $33.1 \pm 2.8$ & $0.86 \pm 0.10$ & $85.5 \pm 20.1$ & Lie16 \\
\hline XRR 080802 & - & 179.50 & $-1.67 \pm 0.09$ & $-0.98 \pm 0.45$ & $123.4 \pm 9.6$ & $0.88 \pm 0.10$ & $63.3 \pm 8.4$ & Lie16 \\
\hline XRR 080903 & - & 66.34 & $-1.66 \pm 0.05$ & $-0.93 \pm 0.22$ & $118.8 \pm 3.7$ & $0.95 \pm 0.07$ & $68.0_{-5.7}^{+17.5}$ & Lie16 \\
\hline XRR 080905B & 2.3739 & 120.94 & $-1.65 \pm 0.07$ & $-1.58_{-0.38}^{+0.28}$ & $179.7 \pm 8.6$ & $0.79 \pm 0.06$ & 256.1 & Lie16 \\
\hline XRR 080915A & - & 26.29 & $-1.58 \pm 0.15$ & $\begin{array}{l}-1.29_{-0.30}^{+1.30} \\
-1.30\end{array}$ & $28.0 \pm 3.9$ & $0.78 \pm 0.10$ & 103.1 & Lie16 \\
\hline XRR 080916A & 0.6887 & 61.35 & $-1.55 \pm 0.02$ & $-1.19 \pm 0.09$ & $421.8 \pm 6.3$ & $0.73 \pm 0.02$ & $121.0_{-12.3}^{+35.2}$ & Lie16 \\
\hline XRR 080928 & 1.6919 & 233.66 & $-1.73 \pm 0.06$ & $-1.73_{-0.24}^{+0.12}$ & $246.0 \pm 6.3$ & $0.82 \pm 0.03$ & - & - \\
\hline XRR 081008 & 1.9683 & 187.82 & $-1.62 \pm 0.04$ & $-1.35 \pm 0.14$ & $414.7 \pm 10.2$ & $0.78 \pm 0.03$ & $118.2_{-18.6}^{+953.0}$ & Lie16 \\
\hline XRR 081017 & - & - & $-1.63 \pm 0.12$ & $-1.51_{-0.18}^{+0.42}$ & $142.4 \pm 11.3$ & $0.77 \pm 0.09$ & 201.7 & Lie16 \\
\hline XRR 081022 & - & 157.58 & $-1.62 \pm 0.06$ & $\begin{array}{r}-1.31_{-0.18}^{+0.10} \\
-0.18\end{array}$ & $251.2 \pm 10.4$ & $0.78 \pm 0.05$ & $108.3 \pm 20.1$ & Lie16 \\
\hline XRR 081028A & 3.038 & 284.42 & $-1.77 \pm 0.04$ & $\begin{array}{r}-0.18 \\
-1.40 \pm 0.16\end{array}$ & $379.6 \pm 11.4$ & $0.88 \pm 0.04$ & $75.2_{-8.6}^{+71.3}$ & Lie16 \\
\hline XRR 081102 & - & 50.32 & $-1.66 \pm 0.06$ & $-1.47_{-0.15}^{+0.26}$ & $222.3 \pm 10.5$ & $0.81 \pm 0.05$ & $129.7 \pm 32.7$ & Lie16 \\
\hline XRR 081104 & - & 64.00 & $-1.94 \pm 0.07$ & $-1.52 \pm 0.27$ & $202.9 \pm 10.2$ & $1.00 \pm 0.08$ & $51.2 \pm 18.4$ & Lie16 \\
\hline XRR 081109A & 0.9787 & 221.49 & $-1.61 \pm 0.04$ & $-1.45_{-0.13}^{+0.17}$ & $394.2 \pm 11.7$ & $0.77 \pm 0.03$ & $169.7 \pm 42.3$ & Lie16 \\
\hline XRR 081118 & 2.58 & 53.40 & $-2.11 \pm 0.08$ & $-1.73_{-0.19}^{+0.33}$ & $113.3 \pm 6.3$ & $1.15 \pm 0.11$ & $29.7_{-14.0}^{+19.9}$ & Lie16 \\
\hline XRR 081127 & - & 17.30 & $-2.04 \pm 0.15$ & $-2.11_{-0.15}^{+0.136}$ & 38.4 & $0.94 \pm 0.32$ & 60.6 & Lie16 \\
\hline XRR 081128 & - & 102.32 & $-1.88 \pm 0.04$ & $\begin{array}{l}-0.94 \pm 0.20 \\
-0.15\end{array}$ & $233.6 \pm 7.9$ & $1.03 \pm 0.05$ & $49.9_{-2.7}^{+4.0}$ & Lie16 \\
\hline XRR 081203B & - & 161.60 & $-1.69 \pm 0.07$ & $-1.69 \pm 0.07$ & $184.4 \pm 15.1$ & $1.00 \pm 0.13$ & - & - \\
\hline XRR 081221 & 2.26 & 33.91 & $-1.75 \pm 0.01$ & $-1.30 \pm 0.06$ & $1888.0 \pm 15.1$ & $0.82 \pm 0.01$ & $90.4_{-3.8}^{+6.0}$ & Lie16 \\
\hline XRR 081230 & 2.03 & 60.69 & $-1.91 \pm 0.08$ & $-1.49_{-0.29}^{+0.34}$ & $82.1 \pm 4.8$ & $1.00 \pm 0.09$ & $52.2 \pm 20.0$ & Lie16 \\
\hline XRR 090107A & - & 12.08 & $-1.64 \pm 0.14$ & $0.29_{-0.71}^{+1.23}$ & $18.9 \pm 2.4$ & $1.10 \pm 0.26$ & $48.8_{-5.2}^{+14.9}$ & Lie16 \\
\hline XRR 090113 & 1.7493 & 9.10 & $-1.53 \pm 0.05$ & $-1.46 \pm 0.12$ & $77.3 \pm 2.4$ & $0.73 \pm 0.03$ & 394.6 & Lie16 \\
\hline XRR 090123 & - & 137.34 & $-1.59 \pm 0.06$ & $-1.57 \pm 0.17$ & $292.6 \pm 11.4$ & $0.75 \pm 0.04$ & - & - \\
\hline XRR 090313 & 3.3736 & 83.04 & $-1.90 \pm 0.14$ & $-1.90_{-0.20}^{+0.46}$ & $146.6 \pm 14.9$ & $0.93 \pm 0.13$ & - & - \\
\hline XRR 090401A & - & 117.00 & $-1.63 \pm 0.02$ & $-1.52 \pm 0.07$ & $1108.0 \pm 15.5$ & $0.77 \pm 0.02$ & $242.7 \pm 58.9$ & Lie16 \\
\hline XRR 090404 & 2.87 & 82.02 & $-2.26 \pm 0.04$ & $-1.94_{-0.08}^{+0.16}$ & $301.5 \pm 7.2$ & $1.24 \pm 0.05$ & $8.0_{-3.6}^{+9.7}$ & Lie16 \\
\hline
\end{tabular}


Table 2-Continued

\begin{tabular}{|c|c|c|c|c|c|c|c|c|}
\hline GRB Name & $\mathrm{z}$ & $\begin{array}{l}T_{90} \\
(\mathrm{~s})\end{array}$ & $\alpha_{P L}$ & $\alpha_{C P L}$ & $\begin{array}{c}S(15-150 \mathrm{keV}) \\
\left(10^{-8} \mathrm{erg} \mathrm{cm}^{-2}\right)\end{array}$ & SR & $\begin{array}{l}E_{\text {peak }}^{o b s} \\
(\mathrm{keV})\end{array}$ & Ref \\
\hline XRR 090407 & 1.4485 & 315.47 & $-1.65 \pm 0.14$ & $-1.59_{-0.66}^{+0.45}$ & $115.1 \pm 11.3$ & 0.78 & 310.0 & Lie16 \\
\hline XRR 090417B & 0.345 & 266.94 & $-1.81 \pm 0.07$ & $\begin{array}{r}-2.30_{-0.07}^{+0.06} \\
-0.07\end{array}$ & $190.8 \pm 10.7$ & $1.17 \pm 0.23$ & 112.9 & Lie16 \\
\hline XRR 090418B & - & 38.00 & $-1.68 \pm 0.02$ & $-1.55 \pm 0.06$ & $1640.9 \pm 14.2$ & $0.80 \pm 0.01$ & $200.9_{-34.6}^{+2417.5}$ & Lie16 \\
\hline XRR 090422 & - & 8.48 & $-1.68 \pm 0.14$ & $-1.71_{-0.13}^{+0.20}$ & $15.9 \pm 2.0$ & $1.00 \pm 0.20$ & $\begin{array}{l}-34.6 \\
-\end{array}$ & - \\
\hline XRR 090423 & 8.26 & 10.30 & $-1.81 \pm 0.05$ & $-0.76 \pm 0.22$ & $62.4 \pm 2.3$ & $0.98 \pm 0.05$ & $53.2_{-2.9}^{+4.7}$ & Lie16 \\
\hline XRR 090426 & 2.609 & 1.24 & $-1.84 \pm 0.11$ & $-1.11_{-0.40}^{+0.50}$ & $17.6 \pm 1.4$ & $0.97 \pm 0.13$ & $55.1 \pm 8.1$ & Lie16 \\
\hline XRR 090429B & 9.38 & 5.58 & $-1.89 \pm 0.06$ & $\begin{array}{r}-0.40 \\
-0.54 \pm 0.34\end{array}$ & $32.9 \pm 1.7$ & $1.15 \pm 0.11$ & $45.3_{-2.7}^{+4.2}$ & Lie16 \\
\hline XRR 090509 & - & 336.38 & $-1.70 \pm 0.10$ & $-1.33 \pm 0.40$ & $320.5 \pm 23.0$ & $0.83 \pm 0.09$ & $88.5 \pm 29.3$ & Lie16 \\
\hline XRR 090516A & 4.109 & 181.01 & $-1.67 \pm 0.06$ & $-1.67_{-0.25}^{+0.14}$ & $928.2 \pm 33.6$ & $0.80 \pm 0.03$ & $315.0 \pm 15.0$ & Gru13 \\
\hline XRR 090529A & 2.625 & 70.44 & $-1.99 \pm 0.08$ & $\begin{array}{l}-0.87_{-0.39}^{+0.49} \\
-0.37\end{array}$ & $93.4 \pm 6.0$ & $1.19 \pm 0.13$ & $42.1_{-4.2}^{+5.7}$ & Lie16 \\
\hline XRR 090530 & 1.266 & 40.46 & $-1.56 \pm 0.08$ & $-1.08 \pm 0.27$ & $104.8 \pm 6.0$ & $0.76 \pm 0.06$ & $92.1 \pm 15.3$ & Lie16 \\
\hline XRR 090531A & - & 42.80 & $-1.58 \pm 0.05$ & $-0.94 \pm 0.20$ & $154.0 \pm 5.8$ & $0.78 \pm 0.04$ & $78.6_{-7.8}^{+26.7}$ & Lie16 \\
\hline XRR 090618 & 0.54 & 113.34 & $-1.58 \pm 0.01$ & $-1.49 \pm 0.04$ & $10890.3 \pm 60.5$ & $0.74 \pm 0.01$ & $162.0 \pm 3.0$ & Gru13 \\
\hline XRR 090621A & - & 219.24 & $-1.67 \pm 0.05$ & $-1.30 \pm 0.19$ & $282.9 \pm 10.0$ & $0.82 \pm 0.04$ & $90.5_{-12.6}^{+450.6}$ & Lie16 \\
\hline XRR 090708 & - & 8.71 & $-1.86 \pm 0.11$ & $-1.17_{-0.39}^{+0.48}$ & $47.3 \pm 3.8$ & $0.99 \pm 0.13$ & $53.0 \pm 8.8$ & Lie16 \\
\hline XRR 090715B & 3 & 266.40 & $-1.58 \pm 0.03$ & $-1.58_{-0.03}^{+0.10}$ & $567.2 \pm 11.8$ & $0.75 \pm 0.02$ & $134.0 \pm 30.0$ & Gru13 \\
\hline XRR 090728 & - & 64.00 & $-1.92 \pm 0.13$ & $\begin{array}{l}-1.56_{-0.32}^{+0.09} \\
-0.52\end{array}$ & $106.2 \pm 9.2$ & $0.98 \pm 0.17$ & $55.8 \pm 27.9$ & Lie16 \\
\hline XRR 090813 & - & 7.14 & $-1.70 \pm 0.06$ & $-1.67 \pm 0.21$ & $132.7 \pm 4.9$ & $0.81 \pm 0.04$ & - & - \\
\hline XRR 090814A & 0.696 & 78.06 & $-1.84 \pm 0.10$ & $-1.02 \pm 0.39$ & $116.4 \pm 8.6$ & $0.99 \pm 0.12$ & $52.4_{-6.3}^{+47.5}$ & Lie16 \\
\hline XRR 090823 & - & 25.60 & $-2.26 \pm 0.15$ & $-2.35_{-0.15}^{+0.93}$ & $19.3 \pm 1.9$ & $1.19 \pm 0.24$ & 159.7 & Lie16 \\
\hline XRR 090904A & - & 129.53 & $-2.01 \pm 0.05$ & $-1.67 \pm 0.17$ & $289.7 \pm 9.8$ & $1.05 \pm 0.05$ & $42.4 \pm 20.4$ & Lie16 \\
\hline XRR 090904B & - & 64.00 & $-1.56 \pm 0.04$ & $-1.56_{-0.04}^{+0.07}$ & $1276.6 \pm 26.9$ & $0.75 \pm 0.02$ & - & - \\
\hline XRR 090912 & - & 135.52 & $-1.68 \pm 0.05$ & $-0.96 \pm 0.20$ & $447.9 \pm 15.5$ & $0.84 \pm 0.04$ & $69.1_{-5.5}^{+14.3}$ & Lie16 \\
\hline XRR 090929B & - & 357.36 & $-1.81 \pm 0.04$ & $-1.64_{-0.12}^{+0.17}$ & $570.3 \pm 16.8$ & $0.89 \pm 0.04$ & $98.1 \pm 21.1$ & Lie16 \\
\hline XRR 091020 & 1.71 & 38.92 & $-1.53 \pm 0.03$ & $-1.45_{-0.07}^{+0.13}$ & $377.9 \pm 7.9$ & $0.72 \pm 0.02$ & $187.0 \pm 25.0$ & Gru13 \\
\hline XRR 091026 & - & 174.00 & $-1.70 \pm 0.07$ & $-1.61 \pm 0.26$ & $200.9 \pm 10.6$ & $0.82 \pm 0.06$ & $183.9 \pm 82.1$ & Lie16 \\
\hline XRR 091029 & 2.752 & 39.18 & $-1.88 \pm 0.03$ & $-1.46 \pm 0.13$ & $240.5 \pm 5.3$ & $0.95 \pm 0.03$ & $61.3_{-4.6}^{+12.5}$ & Lie16 \\
\hline XRR 091104 & - & 107.14 & $-1.73 \pm 0.12$ & $-1.12_{-0.25}^{+0.62}$ & $71.1 \pm 7.1$ & $0.91 \pm 0.13$ & $62.3 \pm 18.7$ & Lie16 \\
\hline XRR 091127 & 0.49044 & 6.96 & $-2.02 \pm 0.04$ & $-1.80 \pm 0.14$ & - & $1.02 \pm 0.06$ & $34.0 \pm 1.0$ & Gru13 \\
\hline XRR 091130B & - & 101.76 & $-2.09 \pm 0.07$ & $-1.52_{-0.24}^{+0.32}$ & $137.3 \pm 7.5$ & $1.19 \pm 0.11$ & $34.1_{-14.3}^{+6.6}$ & Lie16 \\
\hline XRR 091208B & 1.0633 & 14.80 & $-1.76 \pm 0.05$ & $-1.58_{-0.14}^{+0.21}$ & $316.0 \pm 11.1$ & $0.85 \pm 0.05$ & $116.1 \pm 25.5$ & Lie16 \\
\hline XRR 091221 & - & 62.90 & $-1.58 \pm 0.03$ & $-1.55 \pm 0.04$ & $565.7 \pm 8.2$ & $0.75 \pm 0.02$ & - & - \\
\hline XRR 100111A & - & 14.00 & $-1.74 \pm 0.07$ & $-1.71 \pm 0.28$ & $67.4 \pm 3.1$ & $0.84 \pm 0.06$ & 341.0 & Lie16 \\
\hline XRR 100203A & - & 11.20 & $-1.59 \pm 0.18$ & $-1.59_{-0.18}^{+0.60}$ & $24.5 \pm 3.4$ & 0.75 & - & - \\
\hline XRR 100205A & - & 25.84 & $-1.58 \pm 0.12$ & $-1.38_{-0.22}^{+0.56}$ & $35.6 \pm 3.4$ & $0.76 \pm 0.10$ & 142.6 & Lie16 \\
\hline XRR 100213B & 0.604 & 91.86 & $-2.29 \pm 0.08$ & $-2.28 \pm 0.07$ & 122.0 & $1.25 \pm 0.10$ & - & - \\
\hline XRR $100302 \mathrm{~A}$ & 4.813 & 17.95 & $-1.72 \pm 0.10$ & $-1.43 \pm 0.32$ & $30.7 \pm 2.2$ & $0.85 \pm 0.09$ & $90.2 \pm 19.9$ & Lie16 \\
\hline XRR 100316C & - & 9.27 & $-1.57 \pm 0.13$ & $-1.49_{-0.17}^{+0.46}$ & $18.2 \pm 1.5$ & $0.74 \pm 0.09$ & 289.9 & Lie16 \\
\hline XRR 100424A & 2.465 & 104.00 & $-1.83 \pm 0.06$ & $-1.69 \pm 0.22$ & $147.5 \pm 7.0$ & $0.91 \pm 0.06$ & $95.3 \pm 36.9$ & Lie16 \\
\hline XRR 100427A & - & 24.00 & $-1.71 \pm 0.14$ & $-1.00_{-0.46}^{+0.72}$ & $23.6 \pm 2.5$ & $0.87 \pm 0.14$ & $65.5 \pm 10.5$ & Lie16 \\
\hline XRR 100504A & - & 97.00 & $-1.76 \pm 0.05$ & $-1.68 \pm 0.18$ & $232.2 \pm 7.9$ & $0.86 \pm 0.04$ & $179.8 \pm 57.0$ & Lie16 \\
\hline XRR 100513A & 4.772 & 83.50 & $-1.59 \pm 0.07$ & $-1.52 \pm 0.30$ & $142.0 \pm 6.9$ & $0.76 \pm 0.05$ & 321.4 & Lie16 \\
\hline XRR $100514 \mathrm{~A}$ & - & 23.02 & $-1.98 \pm 0.13$ & $-0.91_{-0.52}^{+0.68}$ & $36.4 \pm 3.5$ & $1.17 \pm 0.20$ & $42.5_{-12.6}^{+20.5}$ & Lie16 \\
\hline XRR $100522 \mathrm{~A}$ & - & 48.00 & $-1.90 \pm 0.05$ & $-1.61 \pm 0.17$ & $199.5 \pm 6.8$ & $0.96 \pm 0.05$ & $60.6 \pm 8.0$ & Lie16 \\
\hline XRR 100526A & - & 97.60 & $-1.84 \pm 0.08$ & $-1.84_{-0.49}^{+0.22}$ & $231.3 \pm 14.6$ & $0.93 \pm 0.07$ & - & - \\
\hline
\end{tabular}


Table 2-Continued

\begin{tabular}{|c|c|c|c|c|c|c|c|c|}
\hline GRB Name & $\mathrm{z}$ & $\begin{array}{c}T_{90} \\
(\mathrm{~s})\end{array}$ & $\alpha_{P L}$ & $\alpha_{C P L}$ & $\begin{array}{c}S(15-150 \mathrm{keV}) \\
\left(10^{-8} \mathrm{erg} \mathrm{cm}^{-2}\right)\end{array}$ & $\mathrm{SR}$ & $\begin{array}{l}E_{p e a k}^{o b s} \\
(\mathrm{keV})\end{array}$ & Ref \\
\hline XRR 100614A & - & 225.00 & $-1.86 \pm 0.07$ & $-1.75 \pm 0.26$ & $260.8 \pm 14.1$ & $0.93 \pm 0.07$ & $92.2 \pm 44.5$ & Lie16 \\
\hline XRR 100615A & 1.398 & 38.82 & $-1.86 \pm 0.02$ & $-1.65 \pm 0.09$ & $482.1 \pm 6.4$ & $0.91 \pm 0.02$ & $85.5_{-8.6}^{+54.0}$ & Lie16 \\
\hline XRR 100619A & - & 97.70 & $-1.78 \pm 0.03$ & $-1.74_{-0.05}^{+0.12}$ & $438.9 \pm 7.9$ & $0.86 \pm 0.05$ & $337.7 \pm 145.5$ & Lie16 \\
\hline XRR $100621 \mathrm{~A}$ & 0.542 & 63.55 & $-1.90 \pm 0.01$ & $-1.81 \pm 0.05$ & $2041.9 \pm 17.8$ & $0.93 \pm 0.01$ & $83.0 \pm 9.0$ & Gru13 \\
\hline XRR 100628A & 0.102 & 0.04 & $-1.25 \pm 0.12$ & $2.65_{-0.91}^{+1.85}$ & 0.6 & $0.96 \pm 0.35$ & $74.3_{-4.5}^{+6.1}$ & Lie16 \\
\hline XRR 100702A & - & 0.51 & $-1.61 \pm 0.08$ & $-1.06 \pm 0.31$ & $13.9 \pm 0.8$ & $0.77 \pm 0.07$ & $87.5 \pm 12.7$ & Lie16 \\
\hline XRR 100704A & - & 196.88 & $-1.70 \pm 0.03$ & $-1.65_{-0.04}^{+0.12}$ & $582.1 \pm 11.2$ & $0.81 \pm 0.02$ & 381.8 & Lie16 \\
\hline XRR 100719A & - & 36.00 & $-1.68 \pm 0.13$ & $\begin{array}{r}-1.68_{-0.12}^{+0.043} \\
-0.13\end{array}$ & $53.2 \pm 4.3$ & $0.80 \pm 0.10$ & - & - \\
\hline XRR 100724A & 1.288 & 1.39 & $-1.92 \pm 0.10$ & $-0.51_{-0.52}^{+0.64}$ & $14.1 \pm 1.2$ & $1.24 \pm 0.20$ & $42.5_{-4.1}^{+7.6}$ & Lie16 \\
\hline XRR 100725B & - & 173.70 & $-1.82 \pm 0.03$ & $-1.82 \pm 0.03$ & $653.9 \pm 9.5$ & $0.88 \pm 0.01$ & - & - \\
\hline XRR 100727A & - & 71.90 & $-1.81 \pm 0.06$ & $-1.47 \pm 0.21$ & $115.9 \pm 5.7$ & $0.91 \pm 0.06$ & $69.5 \pm 10.5$ & Lie16 \\
\hline XRR 100728A & 1.567 & 193.38 & $-1.12 \pm 0.01$ & $-1.06 \pm 0.01$ & $1089.4 \pm 26.7$ & $0.99 \pm 0.04$ & - & - \\
\hline XRR 100728B & 2.106 & 12.08 & $-1.54 \pm 0.07$ & $-1.34_{-0.16}^{+0.30}$ & $165.3 \pm 7.6$ & $0.73 \pm 0.06$ & $104.0 \pm 14.0$ & Gru13 \\
\hline XRR 100802A & - & 487.34 & $-1.82 \pm 0.05$ & $-1.40 \pm 0.20$ & $331.7 \pm 13.0$ & $0.92 \pm 0.05$ & $64.6_{-7.4}^{+186.9}$ & Lie16 \\
\hline XRR 100805A & - & 16.74 & $-1.76 \pm 0.12$ & $-1.17_{-0.47}^{+0.54}$ & $48.7 \pm 4.0$ & $0.88 \pm 0.26$ & $68.3 \pm 21.1$ & Lie16 \\
\hline XRR 100823A & - & 17.74 & $-2.17 \pm 0.09$ & $-1.82_{-0.11}^{+0.41}$ & $39.4 \pm 2.7$ & $1.22 \pm 0.14$ & $19.8 \pm 9.6$ & Lie16 \\
\hline XRR 100902A & - & 428.83 & $-1.99 \pm 0.07$ & $\begin{array}{r}-1.97_{-0.18}^{+0.11} \\
-0.18\end{array}$ & $321.5 \pm 13.3$ & $0.99 \pm 0.07$ & 84.8 & Lie16 \\
\hline XRR 100904A & - & 31.25 & $-1.67 \pm 0.08$ & $-1.57_{-0.28}^{+0.31}$ & $125.8 \pm 7.1$ & $0.80 \pm 0.07$ & 199.0 & Lie16 \\
\hline XRR 100906A & 1.727 & 114.63 & $-1.72 \pm 0.02$ & $\begin{array}{r}-1.72 \pm 0.02 \\
-0.28\end{array}$ & $1192.0 \pm 14.3$ & $0.83 \pm 0.02$ & $142.0 \pm 60.0$ & Gru13 \\
\hline XRR 100928A & - & 4.16 & $-1.80 \pm 0.14$ & $-1.37_{-0.45}^{+0.64}$ & $38.0 \pm 3.6$ & $0.88 \pm 0.13$ & 73.3 & Lie16 \\
\hline XRR 101008A & - & 103.50 & $-1.55 \pm 0.12$ & $-1.55 \pm 0.12$ & $116.0 \pm 10.2$ & $0.73 \pm 0.10$ & - & - \\
\hline XRR 101020A & - & 172.34 & $-2.07 \pm 0.09$ & $-1.61_{-0.26}^{+0.44}$ & $243.1 \pm 16.0$ & $1.14 \pm 0.12$ & $35.2 \pm 13.7$ & Lie16 \\
\hline XRR 101024A & - & 18.69 & $-1.83 \pm 0.04$ & $-1.15 \pm 0.15$ & $145.4 \pm 3.6$ & $0.92 \pm 0.03$ & $60.3_{-3.5}^{+6.1}$ & Lie16 \\
\hline XRR 101030A & - & 98.67 & $-1.75 \pm 0.04$ & $-1.75 \pm 0.04$ & $299.4 \pm 7.7$ & $0.84 \pm 0.03$ & - & - \\
\hline XRR 101219B & 0.55185 & 41.86 & $-1.77 \pm 0.13$ & $-0.88_{-0.50}^{+0.68}$ & $236.7 \pm 24.6$ & $0.96 \pm 0.16$ & $54.8 \pm 7.8$ & Lie16 \\
\hline XRR 101225A & 0.847 & - & $-1.83 \pm 0.15$ & $-1.45_{-0.38}^{+0.67}$ & $198.4 \pm 25.3$ & $0.97 \pm 0.20$ & $57.0 \pm 24.9$ & Lie16 \\
\hline XRR 110106A & - & 3.00 & $-1.57 \pm 0.14$ & $-1.24_{-0.58}^{+0.63}$ & $23.3 \pm 2.3$ & $0.76 \pm 0.12$ & 110.9 & Lie16 \\
\hline XRR 110106B & 0.618 & 43.42 & $-1.82 \pm 0.06$ & $-1.67 \pm 0.23$ & $202.0 \pm 8.9$ & $0.90 \pm 0.06$ & 102.0 & Lie16 \\
\hline XRR 110107A & - & 77.00 & $-1.60 \pm 0.03$ & $-1.50 \pm 0.06$ & $556.1 \pm 8.6$ & $0.76 \pm 0.02$ & $280.1 \pm 82.9$ & Lie16 \\
\hline XRR 110205A & 2.22 & 249.42 & $-1.67 \pm 0.02$ & $-1.59 \pm 0.06$ & $1571.7 \pm 21.3$ & $0.80 \pm 0.02$ & $263.3 \pm 84.1$ & Lie16 \\
\hline XRR 110208A & - & 15.98 & $-1.94 \pm 0.13$ & $-1.02_{-0.51}^{+0.68}$ & $21.1 \pm 2.1$ & $1.13 \pm 0.20$ & 43.7 & Lie16 \\
\hline XRR 110210A & - & 192.89 & $-1.77 \pm 0.15$ & $-1.73_{-0.52}^{+0.62}$ & $87.5 \pm 9.5$ & $0.86 \pm 0.14$ & 253.1 & Lie16 \\
\hline XRR $110212 \mathrm{~A}$ & - & 3.70 & $-1.94 \pm 0.08$ & $\begin{array}{l}-0.79_{-0.38}^{+0.47} \\
\end{array}$ & $23.9 \pm 1.5$ & $1.15 \pm 0.12$ & $44.2_{-3.7}^{+6.1}$ & Lie16 \\
\hline XRR 110213A & 1.4607 & 48.00 & $-1.83 \pm 0.06$ & $\begin{array}{r}-1.83_{-0.38}^{+0.38} \\
\end{array}$ & $599.7 \pm 23.2$ & $0.89 \pm 0.04$ & $98.4 \pm 8.5$ & Gru13 \\
\hline XRR 110223A & - & 7.00 & $-1.58 \pm 0.16$ & $-0.67_{-0.65}^{+0.89}$ & $15.2 \pm 2.1$ & $0.84 \pm 0.17$ & $65.2 \pm 10.7$ & Lie16 \\
\hline XRR 110223B & - & 61.45 & $-1.65 \pm 0.10$ & $-1.65 \pm 0.11$ & $61.7 \pm 4.1$ & $1.14 \pm 0.13$ & - & - \\
\hline XRR 110305A & - & 12.00 & $-1.59 \pm 0.10$ & $-1.30_{-0.19}^{+0.38}$ & $78.1 \pm 5.3$ & $0.76 \pm 0.07$ & 120.3 & Lie16 \\
\hline XRR 110315A & - & 87.68 & $-1.77 \pm 0.04$ & $-1.66_{-0.09}^{+0.16}$ & $404.2 \pm 10.3$ & $0.85 \pm 0.03$ & $155.6 \pm 40.7$ & Lie16 \\
\hline XRR 110318A & - & 15.96 & $-1.58 \pm 0.03$ & $-1.06 \pm 0.11$ & $492.8 \pm 9.1$ & $0.75 \pm 0.02$ & $94.6_{-7.4}^{+16.7}$ & Lie16 \\
\hline XRR $110402 \mathrm{~A}$ & - & 56.21 & $-1.56 \pm 0.08$ & $-1.29_{-0.21}^{+0.38}$ & $319.0 \pm 18.0$ & $0.74 \pm 0.07$ & $132.7 \pm 32.3$ & Lie16 \\
\hline XRR 110407A & - & 155.57 & $-1.73 \pm 0.06$ & $-0.74 \pm 0.30$ & $172.4 \pm 8.7$ & $0.92 \pm 0.07$ & $57.8_{-4.4}^{+10.9}$ & Lie16 \\
\hline XRR 110411A & - & 79.40 & $-2.04 \pm 0.04$ & $-1.50 \pm 0.15$ & $329.5 \pm 8.1$ & $1.08 \pm 0.04$ & $41.9_{-4.5}^{+3.5}$ & Lie16 \\
\hline XRR $110412 \mathrm{~A}$ & - & 24.00 & $-1.54 \pm 0.07$ & $-0.78 \pm 0.29$ & $166.3 \pm 9.3$ & $0.78 \pm 0.06$ & $74.8_{-8.5}^{+4.8}$ & Lie16 \\
\hline XRR 110414A & - & 153.12 & $-1.67 \pm 0.07$ & $-1.66_{-0.41}^{+0.23}$ & $350.0 \pm 15.8$ & $0.79 \pm 0.07$ & - & - \\
\hline XRR 110420A & - & 13.87 & $-2.27 \pm 0.03$ & $-1.99_{-0.01}^{+0.08}$ & 640.0 & $1.20 \pm 0.04$ & $2.4 \pm 1.0$ & Lie16 \\
\hline
\end{tabular}


Table 2-Continued

\begin{tabular}{|c|c|c|c|c|c|c|c|c|}
\hline GRB Name & $\mathrm{z}$ & $\begin{array}{r}T_{90} \\
(\mathrm{~s})\end{array}$ & $\alpha_{P L}$ & $\alpha_{C P L}$ & $\begin{array}{c}S(15-150 \mathrm{keV}) \\
\left(10^{-8} \mathrm{erg} \mathrm{cm}^{-2}\right)\end{array}$ & $\mathrm{SR}$ & $\begin{array}{l}E_{p e a k}^{o b s} \\
(\mathrm{keV})\end{array}$ & Ref \\
\hline XRR 110530A & - & 55.73 & $-1.87 \pm 0.14$ & -1.88 & $41.4 \pm 5.0$ & $0.89 \pm 0.13$ & - & - \\
\hline XRR 110715A & 0.8224 & 13.00 & $-1.63 \pm 0.01$ & $-1.25 \pm 0.06$ & 877.7 & 0.96 & $119.8_{-7.5}^{+13.3}$ & Lie16 \\
\hline XRR 110719A & - & 41.23 & $-1.74 \pm 0.08$ & $-1.40_{-0.23}^{+0.34}$ & $184.8 \pm 10.9$ & $0.86 \pm 0.08$ & $81.3 \pm 11.2$ & Lie16 \\
\hline XRR 110726A & 1.036 & 5.16 & $-1.86 \pm 0.09$ & $\begin{array}{l}-0.64_{-0.39}^{+0.48} \\
\end{array}$ & $21.8 \pm 1.6$ & $1.11 \pm 0.14$ & $46.5_{-4.1}^{+7.8}$ & Lie16 \\
\hline XRR 110801A & 1.858 & 385.26 & $-1.82 \pm 0.05$ & $-1.61_{-0.15}^{+0.20}$ & $445.2 \pm 16.0$ & $0.90 \pm 0.05$ & $83.5 \pm 14.7$ & Lie16 \\
\hline XRR 110808A & 1.348 & 40.70 & $-2.11 \pm 0.18$ & $-1.35_{-0.53}^{+1.30}$ & $33.6 \pm 5.0$ & $1.31 \pm 0.56$ & 31.8 & Lie16 \\
\hline XRR 110818A & 3.36 & 102.84 & $-1.58 \pm 0.05$ & $\begin{array}{r}-1.47_{-0.11}^{+0.53} \\
-0.23\end{array}$ & $396.1 \pm 13.4$ & $0.75 \pm 0.11$ & 247.5 & Lie16 \\
\hline XRR 110820A & - & 264.62 & $-1.99 \pm 0.14$ & $-1.99_{-0.51}^{+0.44}$ & $82.2 \pm 6.6$ & $1.00 \pm 0.12$ & - & - \\
\hline XRR 110921A & - & 32.55 & $-1.64 \pm 0.08$ & $\begin{array}{l}-1.54_{-0.55}^{+0.21} \\
\end{array}$ & $216.2 \pm 11.7$ & $0.78 \pm 0.07$ & 213.5 & Lie16 \\
\hline XRR 111008A & 4.9907 & 62.85 & $-1.82 \pm 0.04$ & $-1.70 \pm 0.17$ & $514.7 \pm 14.7$ & $0.89 \pm 0.04$ & $122.6 \pm 29.7$ & Lie16 \\
\hline XRR 111016A & - & 549.00 & $-1.91 \pm 0.06$ & $-1.91 \pm 0.12$ & $390.6 \pm 14.7$ & $0.93 \pm 0.16$ & - & - \\
\hline XRR $111022 \mathrm{~A}$ & - & 22.82 & $-1.73 \pm 0.04$ & $-0.93 \pm 0.18$ & $191.7 \pm 5.9$ & $0.88 \pm 0.09$ & $63.7_{-4.0}^{+8.2}$ & Lie16 \\
\hline XRR 111022B & - & 83.03 & $-1.65 \pm 0.10$ & $-1.52_{-0.17}^{+0.44}$ & $88.5 \pm 7.1$ & $0.80 \pm 0.09$ & 165.6 & Lie16 \\
\hline XRR 111026A & - & 3.58 & $-1.68 \pm 0.10$ & $-1.09_{-0.36}^{+0.52}$ & $15.7 \pm 1.3$ & $0.86 \pm 0.10$ & $69.4 \pm 11.2$ & Lie16 \\
\hline XRR 111109A & - & 13.00 & $-1.86 \pm 0.13$ & $-1.06_{-0.53}^{+0.70}$ & $22.1 \pm 2.5$ & $1.06 \pm 0.18$ & $47.4 \pm 23.6$ & Lie16 \\
\hline XRR 111121A & - & 113.33 & $-1.61 \pm 0.06$ & $-1.61_{-0.06}^{+0.19}$ & $215.1 \pm 7.4$ & $0.76 \pm 0.05$ & $1780.0 \pm 300.0$ & Gru13 \\
\hline XRR 111123A & 3.1516 & 290.00 & $-1.66 \pm 0.03$ & $-1.64_{-0.04}^{+0.08}$ & $715.6 \pm 12.9$ & $0.79 \pm 0.02$ & - & - \\
\hline XRR 111201A & - & 20.80 & $-1.67 \pm 0.15$ & $-1.68 \pm 0.17$ & 29.3 & $0.99 \pm 0.21$ & - & - \\
\hline XRR $111204 \mathrm{~A}$ & - & 48.00 & $-1.83 \pm 0.15$ & $-1.83_{-0.62}^{+0.55}$ & $47.0 \pm 4.5$ & $0.89 \pm 0.14$ & - & - \\
\hline XRR 111208A & - & 168.00 & $-1.55 \pm 0.15$ & $-0.78_{-0.48}^{+0.70}$ & $93.0 \pm 9.3$ & $0.73 \pm 0.12$ & $84.3 \pm 15.2$ & Lie16 \\
\hline XRR $111212 \mathrm{~A}$ & - & 56.30 & $-1.63 \pm 0.09$ & $\begin{array}{l}-0.88_{-0.40}^{+0.40} \\
-0.50\end{array}$ & $130.6 \pm 9.4$ & $0.82 \pm 0.09$ & $71.1 \pm 9.4$ & Lie16 \\
\hline XRR 111215A & - & 373.82 & $-1.60 \pm 0.10$ & $\begin{array}{r}-1.16_{-0.22}^{+0.40} \\
-0.43\end{array}$ & $260.8 \pm 20.5$ & $0.79 \pm 0.09$ & $87.9 \pm 17.0$ & Lie16 \\
\hline XRR 111225A & 0.297 & 105.73 & $-1.75 \pm 0.08$ & $-1.30_{-0.26}^{+0.36}$ & $119.3 \pm 7.5$ & $0.89 \pm 0.08$ & $68.2 \pm 9.6$ & Lie16 \\
\hline XRR 111228A & 0.71627 & 101.24 & $-2.22 \pm 0.03$ & $\begin{array}{l}-1.99 \\
-0.20\end{array}$ & $805.9 \pm 12.0$ & $1.16 \pm 0.03$ & $33.8 \pm 4.1$ & Zan16 \\
\hline XRR 111229A & 1.3805 & 25.37 & $-1.83 \pm 0.17$ & $-1.80 \pm 0.68$ & $29.4 \pm 4.2$ & $0.96 \pm 0.07$ & 289.8 & Lie16 \\
\hline XRR 120102A & - & 38.71 & $-1.59 \pm 0.03$ & $-1.59 \pm 0.03$ & $432.8 \pm 7.1$ & $0.75 \pm 0.02$ & $291.0 \pm 33.0$ & Gru13 \\
\hline XRR 120106A & - & 63.50 & $-1.53 \pm 0.08$ & $-1.32 \pm 0.18$ & $97.1 \pm 6.0$ & $0.73 \pm 0.07$ & 154.3 & Lie16 \\
\hline XRR 120118B & 2.943 & 20.30 & $-2.07 \pm 0.05$ & $-1.59 \pm 0.21$ & $167.4 \pm 6.4$ & $1.12 \pm 0.07$ & $36.9_{-13.9}^{+5.9}$ & Lie16 \\
\hline XRR 120215A & - & 7.92 & $-1.68 \pm 0.10$ & $-1.26_{-0.21}^{+0.48}$ & $24.5 \pm 2.0$ & $0.84 \pm 0.10$ & $78.6 \pm 12.9$ & Lie16 \\
\hline XRR 120219A & 1.728 & 92.53 & $-1.54 \pm 0.11$ & $0.39_{-0.63}^{+0.91}$ & $56.5 \pm 5.9$ & $0.96 \pm 0.16$ & $53.5_{-4.8}^{+12.3}$ & Lie16 \\
\hline XRR 120308A & - & 61.26 & $-1.72 \pm 0.06$ & $-1.10 \pm 0.25$ & $109.4 \pm 5.6$ & $0.88 \pm 0.07$ & $65.0_{-7.1}^{+40.3}$ & Lie16 \\
\hline XRR 120311B & - & 29.69 & $-1.94 \pm 0.07$ & $-1.24 \pm 0.30$ & $104.7 \pm 5.3$ & $0.98 \pm 0.09$ & $44.9_{-5.1}^{+13.6}$ & Lie16 \\
\hline XRR 120312A & - & 14.16 & $-1.71 \pm 0.12$ & $-1.19_{-0.36}^{+0.62}$ & $54.8 \pm 4.5$ & $0.83 \pm 0.11$ & $78.2 \pm 13.4$ & Lie16 \\
\hline XRR 120320A & - & 24.98 & $-1.57 \pm 0.09$ & $-0.31 \pm 0.42$ & $55.3 \pm 4.1$ & $0.82 \pm 0.09$ & $63.3_{-6.0}^{+17.9}$ & Lie16 \\
\hline XRR 120326A & 1.798 & 69.48 & $-1.99 \pm 0.04$ & $-1.40 \pm 0.15$ & $251.7 \pm 6.2$ & $1.05 \pm 0.04$ & $46.8_{-3.3}^{+3.9}$ & Lie16 \\
\hline XRR 120328A & - & 29.93 & $-1.87 \pm 0.10$ & $-0.88_{-0.45}^{+0.58}$ & $42.5 \pm 3.6$ & $1.09 \pm 0.15$ & $46.6_{-5.2}^{+30.3}$ & Lie16 \\
\hline XRR 120401A & 4.5 & 130.27 & $-1.71 \pm 0.11$ & $-1.16_{-0.37}^{+0.45}$ & $87.4 \pm 7.9$ & $0.87 \pm 0.12$ & $68.5 \pm 21.3$ & Lie16 \\
\hline XRR 120403A & - & 1.40 & $-1.59 \pm 0.14$ & $-0.42_{-0.57}^{+0.76}$ & $10.0 \pm 1.1$ & $0.81 \pm 0.14$ & $64.9_{-8.8}^{+123.4}$ & Lie16 \\
\hline XRR 120403B & - & 7.28 & $-1.79 \pm 0.16$ & $-1.30_{-0.39}^{+0.61}$ & $20.0 \pm 2.3$ & $0.91 \pm 0.06$ & $65.6 \pm 25.2$ & Lie16 \\
\hline XRR 120404A & 2.876 & 38.72 & $-1.85 \pm 0.07$ & $\begin{array}{l}-1.81_{-0.32}^{+0.39} \\
-0.35 \\
\end{array}$ & $157.1 \pm 6.5$ & $0.91 \pm 0.06$ & 226.3 & Lie16 \\
\hline XRR $120422 \mathrm{~A}$ & 0.28253 & 60.35 & $-1.99 \pm 0.22$ & $\begin{array}{l}-2.24_{-0.06}^{+1.32} \\
\end{array}$ & $30.1 \pm 7.2$ & $1.14 \pm 0.35$ & 97.0 & Lie16 \\
\hline XRR 120514A & - & 164.34 & $-1.57 \pm 0.05$ & $-1.43_{-0.12}^{+0.20}$ & $277.0 \pm 9.2$ & $0.74 \pm 0.10$ & 209.8 & Lie16 \\
\hline XRR $120521 \mathrm{C}$ & 5.93 & 27.07 & $-1.66 \pm 0.06$ & $-1.36_{-0.18}^{+0.123}$ & $116.8 \pm 4.8$ & $0.81 \pm 0.05$ & $103.0 \pm 18.8$ & Lie16 \\
\hline XRR 120701A & - & 14.16 & $-0.97 \pm 0.05$ & $-0.90_{-0.12}^{+0.18}$ & 16.1 & $0.97 \pm 0.30$ & - & - \\
\hline XRR 120703A & - & 51.70 & $-1.59 \pm 0.04$ & $-1.49_{-0.07}^{+0.15}$ & $375.5 \pm 8.9$ & $0.75 \pm 0.03$ & 262.2 & Lie16 \\
\hline
\end{tabular}


Table 2-Continued

\begin{tabular}{|c|c|c|c|c|c|c|c|c|}
\hline GRB Name & $\mathrm{z}$ & $\begin{array}{l}T_{90} \\
(\mathrm{~s})\end{array}$ & $\alpha_{P L}$ & $\alpha_{C P L}$ & $\begin{array}{c}S(15-150 \mathrm{keV}) \\
\left(10^{-8} \mathrm{erg} \mathrm{cm}^{-2}\right)\end{array}$ & $\mathrm{SR}$ & $\begin{array}{l}E_{\text {peak }}^{o b s} \\
(\mathrm{keV})\end{array}$ & Ref \\
\hline XRR 120711B & - & 60.10 & $-1.71 \pm 0.15$ & $-0.89_{-0.60}^{+1.09}$ & $52.2 \pm 7.7$ & $0.96 \pm 0.21$ & $55.3 \pm 16.6$ & Lie16 \\
\hline XRR 120714A & - & 20.00 & $-1.67 \pm 0.08$ & $\begin{array}{r}-1.13_{-0.30}^{+0.30} \\
\text { +0.36 }\end{array}$ & $85.1 \pm 5.5$ & $0.83 \pm 0.08$ & $75.1 \pm 11.1$ & Lie16 \\
\hline XRR 120714B & 0.3984 & 157.31 & $-1.53 \pm 0.08$ & $-0.69_{-0.34}^{+0.40}$ & $114.9 \pm 8.0$ & $0.79 \pm 0.19$ & $71.2_{-8.6}^{+50.9}$ & Lie16 \\
\hline XRR 120722A & 0.9586 & 36.32 & $-2.01 \pm 0.12$ & $-2.29 \pm 0.33$ & $123.7 \pm 13.8$ & $0.92 \pm 0.33$ & 118.7 & Lie16 \\
\hline XRR 120728A & - & 20.98 & $-1.77 \pm 0.06$ & $-1.39 \pm 0.22$ & $233.9 \pm 9.8$ & $0.86 \pm 0.06$ & $80.9 \pm 11.6$ & Lie16 \\
\hline XRR 120729A & 0.8 & 93.93 & $-1.61 \pm 0.04$ & $-1.61_{-0.04}^{+0.14}$ & $251.1 \pm 6.5$ & $0.76 \pm 0.03$ & $175.9 \pm 17.6$ & Can17 \\
\hline XRR $120802 \mathrm{~A}$ & 3.796 & 50.29 & $-1.85 \pm 0.05$ & $\begin{array}{r}-0.04 \\
-1.22 \pm 0.22\end{array}$ & $164.2 \pm 6.2$ & $0.95 \pm 0.05$ & $57.3_{-4.7}^{+15.0}$ & Lie16 \\
\hline XRR 120807A & - & 19.88 & $-1.83 \pm 0.15$ & $-1.84_{-0.15}^{+0.37}$ & $29.6 \pm 3.1$ & $1.02 \pm 0.25$ & $\begin{array}{l}-4.1 \\
-\end{array}$ & - \\
\hline XRR 120811A & - & 29.01 & $-1.96 \pm 0.07$ & $-1.96 \pm 0.07$ & $75.3 \pm 3.1$ & $0.97 \pm 0.06$ & - & - \\
\hline XRR $120811 C$ & 2.671 & 24.34 & $-1.99 \pm 0.03$ & $-1.33 \pm 0.13$ & $284.5 \pm 5.7$ & $1.05 \pm 0.03$ & $47.3_{-2.4}^{+2.6}$ & Lie16 \\
\hline XRR 120817A & - & 28.21 & $-1.97 \pm 0.08$ & $-1.82_{-0.19}^{+0.34}$ & $69.5 \pm 3.1$ & $1.00 \pm 0.08$ & $\begin{array}{c}-2.4 \\
53.2 \pm 25.0\end{array}$ & Lie16 \\
\hline XRR 120819A & - & 71.02 & $-1.48 \pm 0.11$ & $\begin{array}{l}-0.51_{-0.52}^{+0.19} \\
\end{array}$ & $129.6 \pm 11.7$ & $0.73 \pm 0.09$ & $76.1 \pm 10.7$ & Lie16 \\
\hline XRR 120907A & 0.97 & 6.08 & $-1.65 \pm 0.09$ & $-0.80_{-0.42}^{+0.56}$ & $54.1 \pm 4.3$ & $0.87 \pm 0.10$ & $63.0_{-7.8}^{+196.8}$ & Lie16 \\
\hline XRR 120911A & - & 21.47 & $-1.72 \pm 0.08$ & $-1.07_{-0.35}^{+0.45}$ & $99.7 \pm 6.8$ & $0.89 \pm 0.09$ & $63.5 \pm 8.3$ & Lie16 \\
\hline XRR 120913A & - & 36.04 & $-2.18 \pm 0.07$ & $-2.17 \pm 0.11$ & $83.6 \pm 4.5$ & $1.19 \pm 0.10$ & $26.0 \pm 4.0$ & Gru13 \\
\hline XRR 120913B & - & 122.59 & $-1.63 \pm 0.02$ & $-1.54 \pm 0.06$ & $1101.9 \pm 13.9$ & $0.77 \pm 0.02$ & 271.2 & Lie16 \\
\hline XRR 120918A & - & 25.10 & $-1.60 \pm 0.05$ & $-1.00 \pm 0.19$ & $298.1 \pm 9.6$ & $0.77 \pm 0.02$ & $85.9_{-8.4}^{+28.7}$ & Lie16 \\
\hline XRR $120922 \mathrm{~A}$ & 3.1 & 168.22 & $-2.00 \pm 0.04$ & $-1.58 \pm 0.16$ & $530.8 \pm 14.5$ & $1.04 \pm 0.04$ & $46.1_{-6.8}^{+7.5}$ & Lie16 \\
\hline XRR 120923A & 7.84 & 26.08 & $-1.80 \pm 0.12$ & $0.30_{-0.69}^{+0.99}$ & $32.2 \pm 3.1$ & $1.28 \pm 0.24$ & $44.5_{-3.5}^{+6.8}$ & Lie16 \\
\hline XRR 120927A & - & 35.06 & $-1.63 \pm 0.04$ & $\begin{array}{c}-0.69 \\
-0.66 \pm 0.18\end{array}$ & $231.3 \pm 6.8$ & $0.81 \pm 0.03$ & $67.8_{-3.8}^{+6.5}$ & Lie16 \\
\hline XRR 121014A & - & 80.00 & $-1.87 \pm 0.09$ & $-1.87_{-0.33}^{+0.11}$ & $107.2 \pm 6.6$ & $0.91 \pm 0.05$ & -3.0 & - \\
\hline XRR 121017A & - & 4.61 & $-1.75 \pm 0.09$ & $-1.29_{-0.29}^{+0.41}$ & $62.7 \pm 4.2$ & $0.88 \pm 0.09$ & $69.9 \pm 9.8$ & Lie16 \\
\hline XRR $121027 \mathrm{~A}$ & 1.773 & 80.09 & $-1.81 \pm 0.05$ & $-1.58_{-0.17}^{+0.29}$ & $194.6 \pm 8.6$ & $0.96 \pm 0.06$ & $82.5 \pm 16.2$ & Lie16 \\
\hline XRR 121028A & - & 2.88 & $-1.71 \pm 0.08$ & $\begin{array}{r}-1.40_{-0.22}^{+0.36} \\
+0.16\end{array}$ & $32.7 \pm 1.9$ & $0.84 \pm 0.07$ & 91.8 & Lie16 \\
\hline XRR $121102 \mathrm{~A}$ & - & 54.68 & $-1.86 \pm 0.04$ & $-1.73 \pm 0.16$ & $202.3 \pm 6.1$ & $0.92 \pm 0.04$ & $99.6 \pm 21.9$ & Lie16 \\
\hline XRR 121123A & - & 326.09 & $-1.72 \pm 0.02$ & $-0.99 \pm 0.10$ & $1242.7 \pm 18.2$ & $0.84 \pm 0.02$ & $69.9_{-2.7}^{+4.0}$ & Lie16 \\
\hline XRR $121128 \mathrm{~A}$ & 2.2 & 23.43 & $-1.86 \pm 0.02$ & $-1.32 \pm 0.09$ & $583.0 \pm 7.6$ & $0.91 \pm 0.02$ & $64.9_{-2.7}^{+4.2}$ & Lie16 \\
\hline XRR 121201A & 3.385 & 38.00 & $-1.87 \pm 0.08$ & $-1.83 \pm 0.29$ & $71.6 \pm 4.2$ & $0.92 \pm 0.08$ & $192.9 \pm 92.8$ & Lie16 \\
\hline XRR $121202 \mathrm{~A}$ & - & 19.54 & $-1.60 \pm 0.05$ & $-0.90 \pm 0.23$ & $106.4 \pm 4.4$ & $0.79 \pm 0.05$ & $76.0_{-7.5}^{+27.4}$ & Lie16 \\
\hline XRR $121211 \mathrm{~A}$ & 1.023 & 182.70 & $-2.26 \pm 0.13$ & $-2.47_{-0.14}^{+0.45}$ & $116.0 \pm 18.1$ & $1.27 \pm 0.37$ & 182.0 & Lie16 \\
\hline XRR 121217A & 3.1 & 778.09 & $-1.54 \pm 0.04$ & $-1.50_{-0.06}^{+0.14}$ & $616.4 \pm 15.8$ & $0.73 \pm 0.03$ & $183.9 \pm 56.1$ & Lia15 \\
\hline XRR 121226A & - & 1.01 & $-1.50 \pm 0.14$ & $-1.52 \pm 0.14$ & $8.4 \pm 1.5$ & $0.92 \pm 0.24$ & - & - \\
\hline XRR 130102A & - & 132.78 & $-1.55 \pm 0.11$ & $-0.52_{-0.46}^{+0.58}$ & $78.3 \pm 6.6$ & $0.76 \pm 0.09$ & $72.0_{-8.6}^{+72.4}$ & Lie16 \\
\hline XRR 130131A & - & 51.52 & $-2.13 \pm 0.16$ & $-1.51_{-0.30}^{+0.80}$ & $28.7 \pm 3.4$ & $1.25 \pm 0.28$ & $30.8 \pm 12.3$ & Lie16 \\
\hline XRR 130206A & - & 121.00 & $-1.59 \pm 0.08$ & $\begin{array}{l}-1.59_{-0.08}^{+0.28} \\
\text { - }\end{array}$ & $197.8 \pm 9.7$ & $0.75 \pm 0.06$ & $132.6_{-34.0}^{+34.0}$ & Gru13 \\
\hline XRR $130211 \mathrm{~A}$ & - & 30.75 & $-1.85 \pm 0.12$ & $\begin{array}{r}-1.69_{-0.31}^{+0.48} \\
-0.48\end{array}$ & $64.2 \pm 5.7$ & $0.92 \pm 0.13$ & 84.7 & Lie16 \\
\hline XRR 130215A & 0.597 & 66.22 & $-1.61 \pm 0.07$ & $-1.18 \pm 0.24$ & $529.0 \pm 23.8$ & $0.76 \pm 0.06$ & $101.6 \pm 16.9$ & Lie16 \\
\hline XRR 130306A & - & 272.38 & $-1.75 \pm 0.02$ & $-1.56 \pm 0.10$ & $2928.6 \pm 43.5$ & $0.84 \pm 0.02$ & $124.4_{-19.0}^{+1950.3}$ & Lie16 \\
\hline XRR 130313A & - & 0.17 & $-1.51 \pm 0.17$ & $-1.35 \pm 0.75$ & $1.8 \pm 0.3$ & $0.92 \pm 0.23$ & 209.7 & Lie16 \\
\hline XRR 130315A & - & 230.51 & $-1.73 \pm 0.04$ & $-1.34 \pm 0.17$ & $438.7 \pm 12.9$ & $0.84 \pm 0.04$ & $83.6_{-9.9}^{+95.5}$ & Lie16 \\
\hline XRR 130327A & - & 6.37 & $-2.17 \pm 0.16$ & $-2.32_{-0.14}^{+0.50}$ & 22.7 & $1.04 \pm 0.19$ & 134.2 & Lie16 \\
\hline XRR 130418A & 1.218 & 274.92 & $-2.07 \pm 0.08$ & $\begin{array}{r}-1.29_{-0.35}^{+0.140} \\
-0.35\end{array}$ & $162.3 \pm 11.5$ & $1.23 \pm 0.15$ & $36.1_{-17.2}^{+8.0}$ & Lie16 \\
\hline XRR 130427B & 2.78 & 25.90 & $-1.63 \pm 0.08$ & $\begin{array}{r}-0.35 \\
-1.13_{-0.28}^{+0.35}\end{array}$ & $139.6 \pm 8.1$ & $0.80 \pm 0.07$ & $\begin{array}{l}-17.2 \\
82.9 \pm 12.8\end{array}$ & Lie16 \\
\hline XRR $130502 \mathrm{~A}$ & - & 7.57 & $-1.86 \pm 0.13$ & $-1.74_{-0.30}^{+0.55}$ & $42.6 \pm 3.8$ & $0.92 \pm 0.12$ & 99.1 & Lie16 \\
\hline XRR $130504 \mathrm{~A}$ & - & 120.03 & $-1.61 \pm 0.09$ & $-0.96_{-0.34}^{+0.41}$ & $112.6 \pm 7.5$ & $0.80 \pm 0.08$ & $76.1 \pm 10.5$ & Lie16 \\
\hline
\end{tabular}


Table 2-Continued

\begin{tabular}{|c|c|c|c|c|c|c|c|c|}
\hline GRB Name & $\mathrm{z}$ & $\begin{array}{l}T_{90} \\
(\mathrm{~s})\end{array}$ & $\alpha_{P L}$ & $\alpha_{C P L}$ & $\begin{array}{c}S\left(15-150 \mathrm{keV}^{-}\right) \\
\left(10^{-8} \mathrm{erg} \mathrm{cm}^{-2}\right)\end{array}$ & $\mathrm{SR}$ & $\begin{array}{l}E_{p e a k}^{o b s} \\
(\mathrm{keV})\end{array}$ & Ref \\
\hline XRR 130505A & 2.27 & 89.34 & $-1.71 \pm 0.07$ & $-1.71 \pm 0.06$ & $351.9 \pm 13.9$ & $0.81 \pm 0.04$ & $631.0 \pm 31.0$ & Gru13 \\
\hline XRR 130508A & - & 42.91 & $-1.85 \pm 0.12$ & $-1.19_{-0.49}^{+0.82}$ & $56.0 \pm 6.7$ & $1.04 \pm 0.20$ & 48.5 & Lie16 \\
\hline XRR 130514A & 3.6 & 214.19 & $-1.76 \pm 0.03$ & $-1.64 \pm 0.08$ & $891.2 \pm 14.2$ & $0.84 \pm 0.02$ & $165.5 \pm 37.2$ & Lie16 \\
\hline XRR 130515A & - & 0.30 & $-0.77 \pm 0.09$ & $-0.33_{-0.28}^{+0.45}$ & 0.7 & 1.20 & 233.6 & Lie16 \\
\hline XRR 130528A & 1.25 & 640.00 & $-1.55 \pm 0.05$ & $-1.46_{-0.09}^{+0.18}$ & $563.0 \pm 17.5$ & $0.74 \pm 0.03$ & 295.3 & Lie16 \\
\hline XRR 130529A & - & 87.59 & $-1.61 \pm 0.07$ & $-1.20_{-0.24}^{+0.31}$ & $120.2 \pm 7.1$ & $0.80 \pm 0.07$ & $88.9 \pm 15.4$ & Lie16 \\
\hline XRR 130603A & - & 69.13 & $-1.84 \pm 0.06$ & $-1.71_{-0.12}^{+0.23}$ & $176.1 \pm 7.9$ & $0.84 \pm 0.06$ & 104.2 & Lie16 \\
\hline XRR 130604A & 1.06 & 76.28 & $-1.63 \pm 0.07$ & $-1.63 \pm 0.07$ & $157.1 \pm 6.7$ & $0.77 \pm 0.05$ & - & - \\
\hline XRR 130605A & - & 10.18 & $-1.62 \pm 0.09$ & $-0.93_{-0.35}^{+0.42}$ & $217.5 \pm 14.2$ & $0.80 \pm 0.08$ & $75.2_{-10.0}^{+461.7}$ & Lie16 \\
\hline XRR 130606A & 5.9134 & 276.66 & $-1.52 \pm 0.06$ & $-1.29_{-0.15}^{+0.24}$ & $269.7 \pm 12.1$ & $0.73 \pm 0.05$ & $150.3 \pm 36.2$ & Lie16 \\
\hline XRR 130609A & - & 7.06 & $-1.88 \pm 0.06$ & $-1.28 \pm 0.22$ & $54.2 \pm 2.3$ & $0.98 \pm 0.06$ & $53.9_{-5.0}^{+14.8}$ & Lie16 \\
\hline XRR 130623A & - & 17.62 & $-1.80 \pm 0.10$ & $0.82_{-0.62}^{+0.85}$ & $71.8 \pm 5.8$ & $1.19 \pm 0.18$ & $47.1_{-3.0}^{+4.1}$ & Lie16 \\
\hline XRR 130627A & - & 18.05 & $-1.75 \pm 0.13$ & $1.08_{-0.67}^{+0.86}$ & $69.4 \pm 6.8$ & $0.99 \pm 0.17$ & $51.9_{-4.0}^{+5.3}$ & Lie16 \\
\hline XRR 130627B & - & 48.00 & $-1.75 \pm 0.09$ & $\begin{array}{r}-0.90_{-0.39}^{+0.48} \\
-0.48\end{array}$ & $75.8 \pm 5.7$ & $0.94 \pm 0.11$ & $56.9_{-6.4}^{+5.9}$ & Lie16 \\
\hline XRR $130701 \mathrm{~A}$ & 1.155 & 4.38 & $-1.58 \pm 0.02$ & $-0.90 \pm 0.10$ & $437.6 \pm 6.6$ & $0.73 \pm 0.02$ & $89.2_{-4.7}^{+7.7}$ & Lie16 \\
\hline XRR 130719A & - & 146.97 & $-1.68 \pm 0.09$ & $-1.67 \pm 0.09$ & $210.4 \pm 11.7$ & $0.80 \pm 0.07$ & - & - \\
\hline XRR 130725A & - & 101.52 & $-1.60 \pm 0.15$ & $-1.62_{-0.15}^{+0.30}$ & $98.6 \pm 8.4$ & $0.77 \pm 0.06$ & - & - \\
\hline XRR 130727A & - & 13.56 & $-1.65 \pm 0.02$ & $-1.42 \pm 0.09$ & $378.1 \pm 5.4$ & $0.78 \pm 0.02$ & $137.8_{-19.7}^{+163.1}$ & Lie16 \\
\hline XRR 130803A & - & 43.57 & $-1.65 \pm 0.07$ & $-1.17_{-0.27}^{+0.35}$ & $136.2 \pm 6.7$ & $0.79 \pm 0.06$ & $88.6 \pm 13.4$ & Lie16 \\
\hline XRR 130807A & - & 293.04 & $-1.64 \pm 0.07$ & $-0.92_{-0.29}^{+0.31}$ & $208.5 \pm 10.4$ & $0.81 \pm 0.06$ & $72.7_{-7.7}^{+43.2}$ & Lie16 \\
\hline XRR 130816A & - & 29.98 & $-1.86 \pm 0.17$ & $-1.52_{-0.35}^{+0.91}$ & $39.2 \pm 11.2$ & $0.96 \pm 0.19$ & 59.1 & Lie16 \\
\hline XRR 130816B & - & 8.68 & $-1.83 \pm 0.09$ & $-0.34_{-0.44}^{+0.55}$ & $24.8 \pm 1.8$ & $1.14 \pm 0.14$ & $46.2_{-3.4}^{+6.4}$ & Lie16 \\
\hline XRR $130822 \mathrm{~A}$ & - & 0.04 & $-1.67 \pm 0.15$ & $0.45_{-0.78}^{+1.12}$ & $1.2 \pm 0.1$ & $0.80 \pm 0.15$ & $60.3_{-5.5}^{+13.1}$ & Lie16 \\
\hline XRR 130831A & 0.4791 & 30.19 & $-1.93 \pm 0.02$ & $-1.91_{-0.11}^{+0.09}$ & $649.7 \pm 8.8$ & $0.95 \pm 0.02$ & $67.0 \pm 4.0$ & Gru13 \\
\hline XRR 130831B & - & 37.58 & $-1.75 \pm 0.10$ & $-0.24_{-0.55}^{+0.71}$ & $130.9 \pm 11.0$ & $0.98 \pm 0.13$ & $53.0_{-4.7}^{+13.0}$ & Lie16 \\
\hline XRR 130919A & - & 99.68 & $-1.51 \pm 0.07$ & $-0.39_{-0.34}^{+0.41}$ & $136.2 \pm 7.9$ & $0.77 \pm 0.06$ & $68.5_{-6.0}^{+17.2}$ & Lie16 \\
\hline XRR 130929A & - & 12.24 & $-1.96 \pm 0.09$ & $\begin{array}{l}-0.97_{-0.39}^{+0.48} \\
\end{array}$ & $64.7 \pm 4.6$ & $1.13 \pm 0.14$ & $43.8_{-5.7}^{+9.0}$ & Lie16 \\
\hline XRR 131001A & - & 5.00 & $-1.85 \pm 0.06$ & $-0.91 \pm 0.28$ & $51.6 \pm 2.4$ & $0.99 \pm 0.07$ & $52.5_{-3.8}^{+7.6}$ & Lie16 \\
\hline XRR $131002 \mathrm{~A}$ & - & 56.53 & $-1.62 \pm 0.10$ & $-0.54_{-0.42}^{+0.51}$ & $51.1 \pm 4.2$ & $0.86 \pm 0.11$ & $61.9_{-6.7}^{+29.3}$ & Lie16 \\
\hline XRR 131024A & - & 112.00 & $-1.84 \pm 0.09$ & $-1.44_{-0.35}^{+0.43}$ & $115.4 \pm 8.4$ & $0.95 \pm 0.10$ & $59.2 \pm 10.8$ & Lie16 \\
\hline XRR 131103A & 0.5955 & 15.21 & $-1.79 \pm 0.09$ & $\begin{array}{r}-1.34_{-0.27}^{+0.38} \\
\end{array}$ & $77.5 \pm 5.4$ & $0.92 \pm 0.10$ & $63.4 \pm 10.1$ & Lie16 \\
\hline XRR 131110A & - & 76.80 & $-1.67 \pm 0.05$ & $-1.39 \pm 0.16$ & $336.8 \pm 11.1$ & $0.80 \pm 0.04$ & $109.0 \pm 19.3$ & Lie16 \\
\hline XRR 131117A & 4.042 & 10.88 & $-1.81 \pm 0.09$ & $0.17_{-0.55}^{+0.80}$ & $24.8 \pm 2.0$ & $1.26 \pm 0.20$ & $44.4_{-3.0}^{+5.2}$ & Lie16 \\
\hline XRR 131128A & - & 3.00 & $-1.81 \pm 0.09$ & $-1.11 \pm 0.33$ & $30.4 \pm 1.8$ & $0.92 \pm 0.09$ & $60.9_{-7.2}^{+68.0}$ & Lie16 \\
\hline XRR $131202 \mathrm{~A}$ & - & 30.20 & $-1.54 \pm 0.12$ & $-1.44_{-0.88}^{+0.50}$ & $60.4 \pm 4.9$ & $0.73 \pm 0.09$ & 275.7 & Lie16 \\
\hline XRR 131224B & - & 8.35 & $-2.08 \pm 0.09$ & $-1.05_{-0.41}^{+0.50}$ & $23.5 \pm 1.7$ & $1.31 \pm 0.19$ & $36.3_{-8.6}^{+5.5}$ & Lie16 \\
\hline XRR 140108A & - & 95.23 & $-1.53 \pm 0.02$ & $-1.51_{-0.04}^{+0.08}$ & $714.4 \pm 8.8$ & $0.72 \pm 0.01$ & - & - \\
\hline XRR 140114A & 3.0 & 139.95 & $-2.07 \pm 0.04$ & $\begin{array}{r}-1.80_{-0.16}^{+0.19} \\
+0.19\end{array}$ & $310.4 \pm 9.5$ & $1.08 \pm 0.05$ & $32.5_{-13.9}^{+8.1}$ & Lie16 \\
\hline XRR 140118A & - & 84.09 & $-1.87 \pm 0.07$ & $-1.36_{-0.27}^{+0.32}$ & $243.9 \pm 12.2$ & $0.97 \pm 0.07$ & $55.6 \pm 6.5$ & Lie16 \\
\hline XRR 140211A & - & 109.53 & $-2.07 \pm 0.07$ & $-1.68_{-0.28}^{+0.32}$ & $180.3 \pm 9.5$ & $1.12 \pm 0.10$ & $34.2 \pm 14.3$ & Lie16 \\
\hline XRR 140213A & 1.2076 & 59.93 & $-1.80 \pm 0.02$ & $\begin{array}{r}-0.28 \\
-1.62 \pm 0.08\end{array}$ & $1182.9 \pm 14.0$ & $0.87 \pm 0.02$ & $113.7_{-14.9}^{+158.7}$ & Lie16 \\
\hline XRR 140301A & 1.416 & 27.80 & $-1.96 \pm 0.14$ & $-2.03_{-0.17}^{+0.38}$ & 42.5 & $1.03 \pm 0.31$ & 14.9 & Lie16 \\
\hline XRR $140311 \mathrm{~A}$ & 4.954 & 70.48 & $-1.70 \pm 0.12$ & $-1.48_{-0.22}^{+0.53}$ & $205.9 \pm 16.9$ & $0.82 \pm 0.10$ & 116.4 & Lie16 \\
\hline XRR 140320A & - & 0.51 & $-1.29 \pm 0.18$ & $-1.15_{-1.38}^{+0.59}$ & $2.1 \pm 0.6$ & $0.93 \pm 0.31$ & 340.8 & Lie16 \\
\hline XRR 140331A & 4.65 & 209.66 & $-1.96 \pm 0.15$ & $-1.42_{-0.47}^{+0.90}$ & $66.0 \pm 8.4$ & $1.12 \pm 0.25$ & 40.6 & Lie16 \\
\hline
\end{tabular}


Table 2-Continued

\begin{tabular}{|c|c|c|c|c|c|c|c|c|}
\hline GRB Name & $\mathrm{z}$ & $\begin{array}{l}T_{90} \\
(\mathrm{~s})\end{array}$ & $\alpha_{P L}$ & $\alpha_{C P L}$ & $\begin{array}{c}S(15-150 \mathrm{keV}) \\
\left(10^{-8} \mathrm{erg} \mathrm{cm}^{-2}\right)\end{array}$ & SR & $\begin{array}{l}E_{p e a k}^{o b s} \\
(\mathrm{keV})\end{array}$ & Ref \\
\hline XRR $140412 \mathrm{~A}$ & - & 48.00 & $-2.03 \pm 0.09$ & $-2.27_{-0.07}^{+0.37}$ & $71.7 \pm 4.1$ & $0.93 \pm 0.11$ & 106.3 & Lie16 \\
\hline XRR 140428A & - & 17.42 & $-1.54 \pm 0.13$ & $\begin{array}{l}-0.40_{-0.53}^{+0.70} \\
\end{array}$ & $30.7 \pm 3.3$ & $0.82 \pm 0.13$ & $64.4_{-8.4}^{+98.1}$ & Lie16 \\
\hline XRR 140430A & 1.6 & 173.59 & $-2.01 \pm 0.11$ & $\begin{array}{r}-2.11_{-0.23}^{+0.53} \\
-0.19\end{array}$ & $110.6 \pm 8.9$ & $0.93 \pm 0.24$ & 47.6 & Lie16 \\
\hline XRR $140502 \mathrm{~A}$ & - & 15.12 & $-1.22 \pm 0.07$ & $-1.23 \pm 0.06$ & $23.1 \pm 2.3$ & $1.01 \pm 0.18$ & - & - \\
\hline XRR $140506 \mathrm{~A}$ & 0.889 & 111.10 & $-1.56 \pm 0.08$ & $-1.45_{-0.53}^{+0.29}$ & $265.8 \pm 13.5$ & $0.74 \pm 0.06$ & 271.0 & Lie16 \\
\hline XRR 140509A & - & 23.22 & $-1.59 \pm 0.10$ & $-1.24_{-0.19}^{+0.44}$ & $120.1 \pm 8.4$ & $0.76 \pm 0.08$ & $107.7 \pm 25.4$ & Lie16 \\
\hline XRR 140515A & 6.33 & 23.42 & $-1.78 \pm 0.06$ & $-0.98 \pm 0.28$ & $61.9 \pm 3.2$ & $0.95 \pm 0.07$ & $56.4_{-4.9}^{+15.6}$ & Lie16 \\
\hline XRR 140516A & - & 0.26 & $-1.89 \pm 0.16$ & $0.49_{-0.84}^{+1.37}$ & $2.7 \pm 0.3$ & $1.28 \pm 0.32$ & $44.6_{-4.5}^{+8.0}$ & Lie16 \\
\hline XRR 140518A & 4.707 & 60.52 & $-1.89 \pm 0.06$ & $-0.98 \pm 0.27$ & $106.4 \pm 5.1$ & $1.06 \pm 0.08$ & $\begin{array}{l}47.9_{-3.6}^{+6.3} \\
{ }_{-3}\end{array}$ & Lie16 \\
\hline XRR 140529A & - & 8.14 & $-1.86 \pm 0.05$ & $-1.31 \pm 0.21$ & $216.4 \pm 7.8$ & $0.94 \pm 0.05$ & $60.0_{-5.5}^{+20.1}$ & Lie16 \\
\hline XRR 140607A & - & 94.77 & $-1.74 \pm 0.11$ & $-1.72_{-0.50}^{+0.39}$ & $210.9 \pm 15.0$ & $0.84 \pm 0.09$ & -0.0 & - \\
\hline XRR 140610A & - & 93.25 & $-1.57 \pm 0.03$ & $-1.35 \pm 0.11$ & $581.7 \pm 10.3$ & $0.74 \pm 0.01$ & $152.9_{-25.5}^{+605.0}$ & Lie16 \\
\hline XRR 140614B & - & 49.87 & $-2.05 \pm 0.13$ & $-0.95_{-0.41}^{+0.75}$ & $53.0 \pm 5.1$ & $1.26 \pm 0.23$ & 38.8 & Lie16 \\
\hline XRR 140619A & - & 233.05 & $-1.56 \pm 0.02$ & $\begin{array}{l}-1.53_{-0.02}^{+0.41} \\
-0.02\end{array}$ & $2121.4 \pm 19.8$ & $0.74 \pm 0.01$ & - & - \\
\hline XRR 140626A & - & 16.16 & $-1.89 \pm 0.09$ & $-0.62 \pm 0.40$ & $35.3 \pm 2.4$ & $1.13 \pm 0.13$ & $45.7_{-3.8}^{+6.4}$ & Lie16 \\
\hline XRR 140628A & - & 10.54 & $-1.56 \pm 0.04$ & $-1.15 \pm 0.18$ & $127.5 \pm 4.4$ & $0.76 \pm 0.04$ & $100.1_{-13.9}^{+14.9}$ & Lie16 \\
\hline XRR 140629A & 2.275 & 38.27 & $-1.85 \pm 0.05$ & $-1.33 \pm 0.21$ & $219.8 \pm 8.3$ & $0.94 \pm 0.05$ & $60.9_{-6.0}^{+27.8}$ & Lie16 \\
\hline XRR 140703A & 3.14 & 68.64 & $-1.70 \pm 0.07$ & $-1.70 \pm 0.06$ & $388.2 \pm 15.5$ & $0.81 \pm 0.05$ & - & - \\
\hline XRR 140706A & - & 47.84 & $-1.75 \pm 0.05$ & $-1.43 \pm 0.18$ & $189.8 \pm 6.2$ & $0.86 \pm 0.04$ & $84.7 \pm 11.8$ & Lie16 \\
\hline XRR 140709A & - & 105.19 & $-1.64 \pm 0.03$ & $-1.28 \pm 0.12$ & $542.2 \pm 10.5$ & $0.78 \pm 0.02$ & $103.8_{-11.5}^{+48.6}$ & Lie16 \\
\hline XRR 140710A & 0.558 & 3.00 & $-2.04 \pm 0.11$ & $-0.96_{-0.50}^{+0.64}$ & $20.9 \pm 1.8$ & $1.24 \pm 0.20$ & 39.6 & Lie16 \\
\hline XRR 140713A & - & 6.02 & $-1.84 \pm 0.07$ & $-1.82 \pm 0.23$ & $36.5 \pm 1.8$ & $0.90 \pm 0.07$ & 296.9 & Lie16 \\
\hline XRR 140716A-2 & - & 124.80 & $-1.69 \pm 0.09$ & $-1.43_{-0.21}^{+0.37}$ & $323.1 \pm 17.9$ & $0.81 \pm 0.08$ & $116.7 \pm 45.5$ & Lie16 \\
\hline XRR 140719A & - & 48.00 & $-1.78 \pm 0.14$ & $-0.86_{-0.57}^{+0.71}$ & $41.6 \pm 4.7$ & $0.97 \pm 0.17$ & $53.8 \pm 6.1$ & Lie16 \\
\hline XRR 140719B & - & 54.00 & $-1.72 \pm 0.09$ & $-1.03_{-0.35}^{+0.42}$ & $122.0 \pm 7.9$ & $0.87 \pm 0.09$ & $66.5_{-8.3}^{+423.3}$ & Lie16 \\
\hline XRR 140818B & - & 31.55 & $-1.83 \pm 0.14$ & $\begin{array}{r}-1.76_{-0.44}^{+0.59} \\
\end{array}$ & $64.6 \pm 5.8$ & $0.89 \pm 0.13$ & $163.9 \pm 81.9$ & Lie16 \\
\hline XRR $140824 \mathrm{~A}$ & - & 3.08 & $-1.99 \pm 0.10$ & $\begin{array}{l}-2.03_{-0.04}^{+0.44} \\
+0.51\end{array}$ & $18.4 \pm 1.2$ & $0.98 \pm 0.12$ & 29.8 & Lie16 \\
\hline XRR $140828 \mathrm{~A}$ & - & 19.15 & $-1.73 \pm 0.06$ & $-0.80 \pm 0.29$ & $136.9 \pm 6.7$ & $0.90 \pm 0.07$ & $59.8_{-4.7}^{+12.2}$ & Lie16 \\
\hline XRR 140907A & 1.21 & 80.00 & $-1.65 \pm 0.04$ & $-1.42 \pm 0.13$ & $443.6 \pm 10.2$ & $0.79 \pm 0.03$ & $129.6 \pm 22.9$ & Lie16 \\
\hline XRR 140916A & - & 68.98 & $-2.14 \pm 0.15$ & $-1.13_{-0.52}^{+0.89}$ & $139.8 \pm 14.1$ & $1.26 \pm 0.24$ & $37.0_{-11.8}^{+12.9}$ & Lie16 \\
\hline XRR 140919A & - & 152.30 & $-1.68 \pm 0.06$ & $-1.25_{-0.22}^{+0.25}$ & $597.2 \pm 24.9$ & $0.82 \pm 0.05$ & $86.2 \pm 12.0$ & Lie16 \\
\hline XRR 141015A & - & 11.00 & $-1.85 \pm 0.18$ & $-1.63_{-0.30}^{+1.05}$ & $15.4 \pm 2.3$ & $0.95 \pm 0.24$ & 66.9 & Lie16 \\
\hline XRR 141017A & - & 55.81 & $-1.56 \pm 0.03$ & $-1.03 \pm 0.13$ & $297.6 \pm 6.6$ & $0.74 \pm 0.02$ & $94.1_{-8.2}^{+20.8}$ & Lie16 \\
\hline XRR 141020A & - & 14.90 & $-1.81 \pm 0.10$ & $-1.32_{-0.38}^{+0.48}$ & $41.4 \pm 3.4$ & $0.96 \pm 0.11$ & 59.8 & Lie16 \\
\hline XRR 141109B & - & 220.50 & $-2.18 \pm 0.17$ & -1.94 & $80.5 \pm 9.4$ & $1.18 \pm 0.21$ & 10.2 & Lie16 \\
\hline XRR $141121 \mathrm{~A}$ & 1.47 & 481.00 & $-1.75 \pm 0.07$ & $-1.43_{-0.24}^{+0.32}$ & $417.3 \pm 23.3$ & $0.88 \pm 0.07$ & $79.0 \pm 15.5$ & Lie16 \\
\hline XRR 141130A & - & 62.86 & $-1.56 \pm 0.04$ & $-1.29_{-0.15}^{+0.18}$ & $270.6 \pm 8.2$ & $0.75 \pm 0.03$ & $129.0 \pm 23.8$ & Lie16 \\
\hline XRR 141205A & - & 1.66 & $-1.18 \pm 0.22$ & $3.31 \pm 0.68$ & 1.9 & 1.00 & $54.2_{-5.7}^{+2.2}$ & Lie16 \\
\hline XRR $141212 \mathrm{~A}$ & - & 0.29 & $-1.60 \pm 0.11$ & $-1.15_{-0.22}^{+0.47}$ & $7.2 \pm 0.6$ & $0.77 \pm 0.10$ & 94.9 & Lie16 \\
\hline XRR 141221A & - & 36.82 & $-1.74 \pm 0.04$ & $\begin{array}{r}-0.22 \\
-1.26 \pm 0.18\end{array}$ & $201.9 \pm 6.3$ & $0.87 \pm 0.04$ & $72.8_{-7.2}^{+33.8}$ & Lie16 \\
\hline XRR 150101A & - & 0.06 & $-1.50 \pm 0.92$ & -1.00 & $1.5 \pm 0.2$ & $0.83 \pm 0.20$ & 80.0 & Lie16 \\
\hline XRR 150103A & - & 49.09 & $-1.61 \pm 0.09$ & $0.03_{-0.45}^{+0.56}$ & $82.5 \pm 6.4$ & $0.94 \pm 0.12$ & $54.2_{-4.2}^{+8.6}$ & Lie16 \\
\hline XRR 150110B & - & 10.58 & $-1.89 \pm 0.07$ & $\begin{array}{r}-1.29_{-0.27}^{+0.31} \\
-0.21\end{array}$ & $46.3 \pm 2.4$ & $1.00 \pm 0.08$ & $51.7_{-5.7}^{+36.9}$ & Lie16 \\
\hline XRR 150120A & 0.46 & 1.20 & $-1.81 \pm 0.09$ & $-1.81_{-0.12}^{+0.30}$ & $14.5 \pm 0.9$ & $0.87 \pm 0.08$ & - & - \\
\hline XRR 150203A & - & 24.44 & $-1.81 \pm 0.06$ & $-1.61_{-0.11}^{+0.12}$ & $91.7 \pm 3.6$ & $0.89 \pm 0.05$ & $89.6 \pm 16.1$ & Lie16 \\
\hline
\end{tabular}


Table 2-Continued

\begin{tabular}{|c|c|c|c|c|c|c|c|c|}
\hline GRB Name & $\mathrm{z}$ & $\begin{array}{r}T_{90} \\
(\mathrm{~s})\end{array}$ & $\alpha_{P L}$ & $\alpha_{C P L}$ & $\begin{array}{c}S(15-150 \mathrm{keV}) \\
\left(10^{-8} \mathrm{erg} \mathrm{cm}^{-2}\right)\end{array}$ & SR & $\begin{array}{l}E_{p e a k}^{o b s} \\
(\mathrm{keV})\end{array}$ & Ref \\
\hline XRR $150204 \mathrm{~A}$ & - & 14.00 & $-1.61 \pm 0.05$ & $-1.55_{-0.08}^{+0.18}$ & $87.8 \pm 3.0$ & $0.76 \pm 0.04$ & 367.6 & Lie16 \\
\hline XRR 150213B & - & 209.00 & $-1.74 \pm 0.07$ & $-0.65 \pm 0.37$ & $289.0 \pm 18.6$ & $0.97 \pm 0.10$ & $53.6_{-4.6}^{+12.5}$ & Lie16 \\
\hline XRR $150222 \mathrm{~A}$ & - & 15.84 & $-1.61 \pm 0.04$ & $-1.28 \pm 0.14$ & $211.9 \pm 5.4$ & $0.77 \pm 0.03$ & $110.4_{-15.3}^{+150.4}$ & Lie16 \\
\hline XRR 150301B & 1.5169 & 17.14 & $-1.46 \pm 0.04$ & $-1.35_{-0.08}^{+0.13}$ & 167.5 & 1.03 & 305.9 & Lie16 \\
\hline XRR $150302 \mathrm{~A}$ & - & 23.87 & $-1.69 \pm 0.16$ & $-0.93_{-0.64}^{+1.25}$ & $23.5 \pm 3.5$ & $0.91 \pm 0.19$ & $60.4 \pm 21.1$ & Lie16 \\
\hline XRR 150314A & 1.758 & 14.78 & $-1.08 \pm 0.01$ & $\begin{array}{r}-1.00_{-0.02}^{+0.05} \\
-\end{array}$ & $417.6 \pm 59.1$ & $1.01 \pm 0.07$ & - & - \\
\hline XRR 150318A & - & 87.44 & $-2.06 \pm 0.06$ & $-1.53_{-0.24}^{+0.32}$ & $231.0 \pm 11.3$ & $1.15 \pm 0.09$ & $36.3_{-16.0}^{+7.0}$ & Lie16 \\
\hline XRR 150323A & 0.593 & 149.73 & $-1.74 \pm 0.04$ & $-1.61_{-0.09}^{+0.15}$ & $553.7 \pm 13.5$ & $0.84 \pm 0.03$ & $154.9 \pm 38.1$ & Lie16 \\
\hline XRR $150323 \mathrm{C}$ & - & 159.66 & $-1.94 \pm 0.15$ & $-0.04_{-0.84}^{+1.12}$ & $115.4 \pm 13.1$ & $1.28 \pm 0.27$ & $43.2_{-7.4}^{+11.4}$ & Lie16 \\
\hline XRR 150402A & - & 14.40 & $-2.28 \pm 0.24$ & -1.89 & $13.9 \pm 2.5$ & $1.26 \pm 0.32$ & 10.2 & Lie16 \\
\hline XRR 150413A & 3.139 & 243.60 & $-1.68 \pm 0.07$ & $-1.48_{-0.16}^{+0.27}$ & $421.4 \pm 19.3$ & $0.81 \pm 0.06$ & $128.4 \pm 31.1$ & Lie16 \\
\hline XRR 150428B & - & 130.95 & $-1.81 \pm 0.05$ & $-0.96 \pm 0.22$ & $357.0 \pm 12.9$ & $0.93 \pm 0.05$ & $58.3_{-3.9}^{+7.7}$ & Lie16 \\
\hline XRR 150527A & - & 108.80 & $-1.71 \pm 0.05$ & $-1.41_{-0.17}^{+0.21}$ & $310.7 \pm 11.4$ & $0.84 \pm 0.04$ & $58.6 \pm 1.6$ & Lie16 \\
\hline XRR 150530B & - & 3.00 & $-1.68 \pm 0.10$ & $-0.58_{-0.45}^{+0.57}$ & $15.8 \pm 1.2$ & $0.90 \pm 0.11$ & $91.2_{-22.3}^{+15.1}$ & Lie16 \\
\hline XRR 150607A & - & 25.99 & $-1.59 \pm 0.05$ & $\begin{array}{r}-0.45 \\
-0.98 \pm 0.19\end{array}$ & $229.7 \pm 7.9$ & $0.77 \pm 0.04$ & $84.2_{-8.4}^{+28.5}$ & Lie16 \\
\hline XRR 150615A & - & 32.68 & $-1.80 \pm 0.10$ & $-1.80_{-0.10}^{+0.37}$ & $61.0 \pm 4.2$ & $0.86 \pm 0.11$ & - & - \\
\hline XRR 150616A & - & 608.41 & $-1.66 \pm 0.03$ & $-1.43 \pm 0.11$ & $1930.8 \pm 34.1$ & $0.79 \pm 0.02$ & $131.7_{-20.3}^{+434.1}$ & Lie16 \\
\hline XRR 150626A & - & 97.26 & $-2.03 \pm 0.09$ & $-2.12_{-0.05}^{+0.26}$ & $136.7 \pm 15.1$ & $1.02 \pm 0.16$ & 63.8 & Lie16 \\
\hline XRR 150710A & - & 0.15 & $-0.64 \pm 0.10$ & $-0.40_{-0.21}^{+0.49}$ & 0.1 & 1.00 & 403.7 & Lie16 \\
\hline XRR 150711A & - & 70.96 & $-1.77 \pm 0.04$ & $-1.48 \pm 0.16$ & $418.7 \pm 11.3$ & $0.86 \pm 0.04$ & $89.8 \pm 12.5$ & Lie16 \\
\hline XRR 150716A & - & 42.57 & $-1.53 \pm 0.05$ & $-1.01_{-0.19}^{+0.21}$ & $141.1 \pm 4.9$ & $0.74 \pm 0.04$ & $94.5_{-11.1}^{+57.2}$ & Lie16 \\
\hline XRR $150722 \mathrm{~A}$ & - & 67.31 & $-1.84 \pm 0.13$ & $-1.84_{-0.56}^{+0.17}$ & $51.7 \pm 5.6$ & $0.77 \pm 0.18$ & - & - \\
\hline XRR $150724 \mathrm{~A}$ & - & 280.01 & $-1.99 \pm 0.07$ & $\begin{array}{r}-1.43 \pm 0.32 \\
-1.30\end{array}$ & $200.1 \pm 11.8$ & $1.09 \pm 0.10$ & $42.1 \pm 17.1$ & Lie16 \\
\hline XRR 150728A & - & 0.83 & $-2.02 \pm 0.24$ & $-2.08_{-0.26}^{+0.55}$ & 3.3 & $0.97 \pm 0.13$ & 37.4 & Lie16 \\
\hline XRR 150801B & - & 408.71 & $-1.80 \pm 0.07$ & $-1.80_{-0.07}^{+0.16}$ & $297.8 \pm 12.2$ & 0.86 & - & - \\
\hline XRR 150811A & - & 31.52 & $-2.13 \pm 0.13$ & $-1.94_{-0.03}^{+0.50}$ & $47.5 \pm 4.3$ & $1.11 \pm 0.20$ & $12.5 \pm 5.8$ & Lie16 \\
\hline XRR 150817A & - & 38.00 & $-1.77 \pm 0.02$ & $-1.61 \pm 0.09$ & $586.3 \pm 7.9$ & $0.85 \pm 0.02$ & $123.2_{-18.9}^{+3961.0}$ & Lie16 \\
\hline XRR 150818A & 0.282 & 143.06 & $-1.87 \pm 0.05$ & $-1.88_{-0.04}^{+0.13}$ & $428.5 \pm 14.9$ & $0.92 \pm 0.03$ & $99.8 \pm 10.1$ & Can17 \\
\hline XRR 150819A & - & 51.66 & $-1.73 \pm 0.14$ & $-1.17_{-0.53}^{+0.75}$ & $44.2 \pm 5.0$ & $0.90 \pm 0.15$ & $64.1 \pm 12.3$ & Lie16 \\
\hline XRR 150831B & - & 6.18 & $-1.90 \pm 0.10$ & $\begin{array}{r}-0.52_{-0.49}^{+0.63} \\
-0.67\end{array}$ & $129.1 \pm 9.5$ & $1.02 \pm 0.12$ & $50.7_{-4.6}^{+9.0}$ & Lie16 \\
\hline XRR 150907B & - & 62.02 & $-1.47 \pm 0.09$ & $\begin{array}{r}-0.16_{-0.45}^{+0.49} \\
-0.58\end{array}$ & $111.5 \pm 8.5$ & $0.76 \pm 0.08$ & $67.6_{-6.6}^{+23.6}$ & Lie16 \\
\hline XRR 150911A & - & 7.14 & $-1.93 \pm 0.03$ & $\begin{array}{r}-1.02 \pm 0.12 \\
-0.40\end{array}$ & $206.6 \pm 3.8$ & $1.00 \pm 0.03$ & $51.6_{-1.7}^{+2.1}$ & Lie16 \\
\hline XRR 150925A & - & 122.13 & $-2.19 \pm 0.10$ & $-2.18_{-0.02}^{+0.73}$ & $129.7 \pm 9.4$ & $1.26 \pm 0.17$ & - & - \\
\hline XRR 151001B & - & 131.52 & $-2.13 \pm 0.13$ & -1.96 & $157.0 \pm 10.0$ & $1.20 \pm 0.18$ & 10.5 & Lie16 \\
\hline XRR 151004A & - & 137.27 & $-1.95 \pm 0.11$ & $-1.95_{-0.36}^{+0.46}$ & $102.2 \pm 7.2$ & $0.96 \pm 0.10$ & 356.4 & Lie16 \\
\hline XRR 151021A & 2.33 & 110.06 & $-1.52 \pm 0.02$ & $-1.52 \pm 0.02$ & $2749.3 \pm 26.3$ & 0.72 & - & - \\
\hline XRR 151022A & - & 117.68 & $-1.89 \pm 0.14$ & $-1.66_{-0.38}^{+0.82}$ & $115.3 \pm 12.4$ & $0.97 \pm 0.17$ & 60.2 & Lie16 \\
\hline XRR 151027A & 0.81 & 129.58 & $-1.54 \pm 0.03$ & $-1.55 \pm 0.03$ & $422.6 \pm 17.1$ & $1.00 \pm 0.05$ & - & - \\
\hline XRR 151027B & 4.063 & 80.00 & $-1.82 \pm 0.14$ & $-1.66_{-0.31}^{+0.49}$ & $145.7 \pm 13.2$ & $0.89 \pm 0.13$ & 104.5 & Lie16 \\
\hline XRR 151118A & - & 23.57 & $-1.71 \pm 0.10$ & $-1.28_{-0.20}^{+0.41}$ & $44.9 \pm 3.6$ & $0.87 \pm 0.10$ & $73.4 \pm 10.1$ & Lie16 \\
\hline XRR 151122A & - & 36.80 & $-1.66 \pm 0.08$ & $-1.60 \pm 0.35$ & $45.3 \pm 2.3$ & $0.79 \pm 0.06$ & $288.2 \pm 135.5$ & Lie16 \\
\hline XRR 151205B & - & 1.44 & $-1.60 \pm 0.16$ & $-1.39_{-0.26}^{+0.63}$ & $7.9 \pm 0.9$ & $0.78 \pm 0.13$ & 132.0 & Lie16 \\
\hline XRR 151210A & - & 86.96 & $-1.84 \pm 0.07$ & $\begin{array}{r}-0.20 \\
-1.05 \pm 0.33\end{array}$ & $136.0 \pm 8.5$ & $1.01 \pm 0.10$ & $50.8_{-5.0}^{+23.3}$ & Lie16 \\
\hline XRR 151215A & 2.59 & 17.85 & $-1.99 \pm 0.16$ & $-1.10_{-0.54}^{+1.03}$ & $27.9 \pm 3.9$ & $1.22 \pm 0.32$ & $\begin{array}{l}-5.0 \\
39.1\end{array}$ & Lie16 \\
\hline XRR 151228B & - & 48.00 & $-1.64 \pm 0.04$ & $-1.10 \pm 0.15$ & $232.3 \pm 6.2$ & $0.80 \pm 0.03$ & $82.0_{-7.3}^{+21.5}$ & Lie16 \\
\hline
\end{tabular}


Table 2-Continued

\begin{tabular}{|c|c|c|c|c|c|c|c|c|}
\hline GRB Name & $\mathrm{z}$ & $\begin{array}{r}T_{90} \\
(\mathrm{~s})\end{array}$ & $\alpha_{P L}$ & $\alpha_{C P L}$ & $\begin{array}{c}S(15-150 \mathrm{keV}) \\
\left(10^{-8} \mathrm{erg} \mathrm{cm}^{-2}\right)\end{array}$ & SR & $\begin{array}{l}E_{\text {peak }}^{o b s} \\
(\mathrm{keV})\end{array}$ & Ref \\
\hline XRR 160104A & - & 16.56 & $-1.76 \pm 0.09$ & $-1.02 \pm 0.36$ & $37.7 \pm 2.9$ & $0.93 \pm 0.11$ & $58.0_{-7.4}^{+140.6}$ & Lie16 \\
\hline XRR 160117A & - & 137.00 & $-2.15 \pm 0.06$ & -1.99 & $309.5 \pm 12.1$ & $1.16 \pm 0.06$ & $1.2 \pm 22.2$ & Lie16 \\
\hline XRR 160119A & - & 120.14 & $-1.63 \pm 0.03$ & $-1.63 \pm 0.03$ & $697.5 \pm 10.2$ & $0.78 \pm 0.02$ & - & - \\
\hline XRR 160121A & - & 10.50 & $-1.77 \pm 0.06$ & $-1.05_{-0.27}^{+0.31}$ & $57.5 \pm 2.9$ & $0.92 \pm 0.07$ & $59.6_{-5.6}^{+22.4}$ & Lie16 \\
\hline XRR 160123A & - & 3.95 & $-1.51 \pm 0.08$ & $\begin{array}{r}-0.57_{-0.37}^{+0.27} \\
-0.36\end{array}$ & $32.2 \pm 2.3$ & $0.79 \pm 0.08$ & $69.6_{-8.0}^{+40.4}$ & Lie16 \\
\hline XRR 160203A & 3.52 & 17.44 & $-1.94 \pm 0.11$ & $-1.78_{-0.26}^{+0.47}$ & $97.7 \pm 7.3$ & $0.98 \pm 0.10$ & 58.5 & Lie16 \\
\hline XRR $160225 \mathrm{~A}$ & - & 157.46 & $-1.57 \pm 0.12$ & $-1.16_{-0.31}^{+0.20}$ & $153.8 \pm 13.0$ & $0.75 \pm 0.10$ & $104.1 \pm 38.7$ & Lie16 \\
\hline XRR 160227A & 2.38 & 316.35 & $-1.55 \pm 0.05$ & $\begin{array}{r}-0.31 \\
-0.75 \pm 0.23\end{array}$ & $312.2 \pm 12.7$ & $0.77 \pm 0.04$ & $75.5_{-6.7}^{+18.9}$ & Lie16 \\
\hline XRR 160314A & 0.726 & 8.73 & $-1.53 \pm 0.11$ & $-1.53 \pm 0.11$ & 27.8 & $0.72 \pm 0.08$ & - & - \\
\hline XRR $160321 \mathrm{~A}$ & - & 33.48 & $-1.89 \pm 0.10$ & $-1.15 \pm 0.49$ & $49.2 \pm 4.0$ & $1.06 \pm 0.12$ & $46.8 \pm 6.8$ & Lie16 \\
\hline XRR 160327A & 4.99 & 33.74 & $-1.84 \pm 0.05$ & $-1.84_{-0.05}^{+0.18}$ & $137.0 \pm 3.8$ & $0.89 \pm 0.05$ & - & - \\
\hline XRR $160412 \mathrm{~A}$ & - & 32.18 & $-1.88 \pm 0.04$ & $-1.72 \pm 0.16$ & $190.6 \pm 5.7$ & $0.94 \pm 0.04$ & $80.3 \pm 14.5$ & Lie16 \\
\hline XRR 160417A & - & 14.55 & $-1.93 \pm 0.12$ & $-1.93 \pm 0.41$ & $57.5 \pm 5.4$ & $0.95 \pm 0.13$ & - & - \\
\hline XRR $160425 \mathrm{~A}$ & 0.555 & 304.60 & $-2.18 \pm 0.09$ & $-1.98 \pm 0.28$ & $200.9 \pm 10.5$ & $1.14 \pm 0.13$ & 5.3 & Lie16 \\
\hline XRR $160504 \mathrm{~A}$ & - & 53.96 & $-1.79 \pm 0.09$ & $-1.79_{-0.41}^{+0.17}$ & 58.2 & $0.86 \pm 0.10$ & - & - \\
\hline XRR 160506A & - & 260.53 & $-2.12 \pm 0.12$ & $-1.99_{-0.01}^{+0.41}$ & $136.3 \pm 22.3$ & $1.18 \pm 0.35$ & 3.0 & Lie16 \\
\hline XRR 160519A & - & 35.62 & $-1.97 \pm 0.13$ & $\begin{array}{r}-1.51_{-0.32}^{+0.01} \\
-0.65 \\
\end{array}$ & $112.2 \pm 10.6$ & $1.05 \pm 0.18$ & 45.8 & Lie16 \\
\hline XRR 160525B & - & 0.29 & $-1.90 \pm 0.18$ & $-0.60_{-0.74}^{+1.10}$ & $2.8 \pm 0.4$ & $1.15 \pm 0.29$ & 45.0 & Lie16 \\
\hline XRR 160611A & - & 35.12 & $-1.68 \pm 0.06$ & $-1.53 \pm 0.13$ & $175.6 \pm 7.2$ & $0.80 \pm 0.05$ & 160.0 & Lie16 \\
\hline XRR 160801A & - & 2.85 & $-1.85 \pm 0.11$ & $-1.00_{-0.46}^{+0.57}$ & $16.2 \pm 1.4$ & $1.01 \pm 0.14$ & $51.3 \pm 7.1$ & Lie16 \\
\hline XRR 160804A & 0.736 & 152.74 & $-1.88 \pm 0.03$ & $-1.41 \pm 0.11$ & $1092.7 \pm 17.7$ & $0.93 \pm 0.02$ & $64.2_{-3.6}^{+6.9}$ & Lie16 \\
\hline XRR 160815A & - & 8.57 & $-1.72 \pm 0.05$ & $-0.96 \pm 0.22$ & $139.4 \pm 4.5$ & $0.85 \pm 0.04$ & $67.7_{-4.8}^{+10.8}$ & Lie16 \\
\hline XRR 160819A & - & 67.20 & $-1.70 \pm 0.03$ & $-1.49 \pm 0.11$ & $765.3 \pm 12.2$ & $0.81 \pm 0.02$ & $133.0_{-20.7}^{+432.1}$ & Lie16 \\
\hline XRR $160821 \mathrm{~A}$ & - & 112.64 & $-1.53 \pm 0.03$ & $-1.47_{-0.05}^{+0.10}$ & $718.8 \pm 12.6$ & $0.72 \pm 0.02$ & 390.0 & Lie16 \\
\hline XRR 160821B & 0.16 & 0.48 & $-1.88 \pm 0.07$ & $\begin{array}{l}-0.12_{-0.40}^{+0.48} \\
\end{array}$ & $10.3 \pm 0.6$ & $1.15 \pm 0.12$ & $46.3_{-2.7}^{+3.7}$ & Lie16 \\
\hline XRR 160824A & - & 99.33 & $-1.62 \pm 0.05$ & $\begin{array}{r}-1.60_{-0.06}^{+0.40} \\
-0.16\end{array}$ & $252.9 \pm 10.2$ & $0.77 \pm 0.05$ & -2.1 & - \\
\hline XRR 160826A & - & 52.62 & $-1.55 \pm 0.14$ & $\begin{array}{r}-1.44_{-0.63}^{+0.50} \\
\end{array}$ & $48.0 \pm 5.0$ & $0.74 \pm 0.11$ & 230.5 & Lie16 \\
\hline XRR 161004A & - & 1.32 & $-1.38 \pm 0.15$ & $-1.39_{-0.55}^{+0.14}$ & 4.3 & $0.99 \pm 0.17$ & - & - \\
\hline XRR 161014A & 2.823 & 23.00 & $-1.44 \pm 0.08$ & $-1.18_{-0.17}^{+0.33}$ & $103.7 \pm 15.0$ & $0.94 \pm 0.19$ & 175.2 & Lie16 \\
\hline XRR 161017A & 2.013 & 217.05 & $-1.57 \pm 0.03$ & $\begin{array}{r}-1.57_{-0.03}^{+0.106} \\
\end{array}$ & $536.9 \pm 8.6$ & 0.74 & - & - \\
\hline XRR 161108A & - & 115.84 & $-1.79 \pm 0.08$ & $-1.31_{-0.28}^{+0.34}$ & $105.0 \pm 6.4$ & $0.91 \pm 0.08$ & $65.1 \pm 8.9$ & Lie16 \\
\hline XRR 161113A & - & 43.46 & $-1.75 \pm 0.05$ & $\begin{array}{r}-0.28 \\
-1.71 \pm 0.16\end{array}$ & $153.4 \pm 5.0$ & $0.84 \pm 0.04$ & $355.5 \pm 139.8$ & Lie16 \\
\hline XRR 161117A & 1.549 & 125.70 & -1.83 & $-1.20 \pm 0.06$ & $2021.7 \pm 16.3$ & $0.86 \pm 0.01$ & $73.1_{-1.9}^{+2.4}$ & Lie16 \\
\hline XRR 161117B & - & 152.31 & $-2.11 \pm 0.03$ & $-1.39_{-0.13}^{+0.14}$ & $563.9 \pm 11.4$ & $1.15 \pm 0.04$ & $38.9_{-3.1}^{+2.2}$ & Lie16 \\
\hline XRR 161129A & - & 35.54 & $-1.53 \pm 0.03$ & $-1.40_{-0.08}^{+0.11}$ & $355.4 \pm 6.4$ & $0.72 \pm 0.02$ & $243.4 \pm 60.5$ & Lie16 \\
\hline XRR $161202 \mathrm{~A}$ & - & 118.50 & $-1.57 \pm 0.03$ & $-1.3 \pm 0.11$ & $850.0 \pm 15.1$ & $0.74 \pm 0.02$ & $142.6_{-21.9}^{+207.0}$ & Lie16 \\
\hline
\end{tabular}

Note. $-\alpha_{P L}$ is the power-law photon index in the simple power-law model, and the data are derived from Lien et al. (2016). $\alpha_{C P L}$ is the power-law photon index in the cutoff power-law model, and the data are derived from Lien et al. (2016). $S(15-150 \mathrm{keV})$ is the fluence of (15-150) keV band, and the data are derived from Lien et al. (2016). $S R$ is the fluence ratio of $S(25-50 \mathrm{keV}) / S(50-100 \mathrm{keV})$. Ref is the reference in which we take $E_{\text {peak }}^{o b s}$ values: Ama08 refers to Amati, et al. (2008), Ama09 refers to Amati, et al. (2009), Can07 refers to Cano, et al. (2017), Gru13 refers to Grupe et al. (2013), Lia15 refers to Liang et al. (2015), Lie16 refers to Lien et al. (2016), and Zan16 refers to Zaninoni et al. (2016). 
Table 3. Statistics of the X-Ray Lightcurve Type for XRFs, XRRs, and C-GRBs

\begin{tabular}{lccccc}
\hline \hline Class & Total Number & Type 0 [fraction] & Type I [fraction] & Type II [fraction] & Type III [fraction] \\
\hline XRF & 34 & $5[(14.7 \pm 6.6) \%]$ & $15[(44.1 \pm 11.4) \%]$ & $11[(32.4 \pm 9.8) \%]$ & $3[(8.8 \pm 5.1) \%]$ \\
XRR & 201 & $35[(17.4 \pm 2.9) \%]$ & $62[(30.8 \pm 3.9) \%]$ & $91[(45.3 \pm 4.7) \%]$ & $13[(6.5 \pm 1.8) \%]$ \\
C-GRB & 135 & $34[(25.2 \pm 4.3) \%]$ & $60[(44.4 \pm 5.7) \%]$ & $37[(27.4 \pm 4.5) \%]$ & $4[(3.0 \pm 1.5) \%]$ \\
\hline
\end{tabular}

Table 4. Statistical Results of the Association between XRF/XRR and Supernova

\begin{tabular}{|c|c|c|c|c|c|c|c|c|c|}
\hline Source & $z$ & $E_{\text {peak }}^{o b s}(\mathrm{keV})$ & Supernova & Grade & Type & $E_{K}$ & $\log \left(M^{*} / M_{\odot}\right)$ & $\log \left(Z / Z_{\odot}\right)$ & SFR \\
\hline XRF 050416A & 0.6528 & $14.8_{-7.0}^{+3.6}$ & $\ldots$ & $\mathrm{D}$ & $\cdots$ & 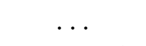 & 9.19 & $\cdots$ & 2.32 \\
\hline XRR 050525A & 0.606 & $80.4_{-1.5}^{+1.7}$ & $2005 \mathrm{nc}$ & B & $\ldots$ & $1.89_{-0.75}^{+1.07}$ & $\ldots$ & $\ldots$ & $\ldots$ \\
\hline XRF 050824 & 0.8278 & 80.0 & $\ldots$ & $\mathrm{E}$ & $\ldots$ & $0.57_{-0.37}^{+0.93}$ & $\ldots$ & -0.3 & $\ldots$ \\
\hline XRF 060218 & 0.03342 & $4.7 \pm 0.3$ & 2006aj & $\mathrm{A}$ & Ic & $1.02 \pm 0.23$ & 7.78 & -0.53 & 0.05 \\
\hline XRR 060729 & 0.5428 & $201.2 \pm 68.9$ & $\ldots$ & $\mathrm{D}$ & $\cdots$ & $2.44_{-0.99}^{+1.43}$ & $\cdots$ & $\ldots$ & $\cdots$ \\
\hline XRR 060904B & 0.7029 & $84.1 \pm 13.3$ & $\ldots$ & $\mathrm{C}$ & $\ldots$ & $0.99_{-0.37}^{+0.51}$ & $\ldots$ & $\ldots$ & $\ldots$ \\
\hline XRF 070419A & 0.9705 & $26.5_{-11.2}^{+3.6}$ & $\ldots$ & $\mathrm{D}$ & $\ldots$ & $\ldots$ & $\ldots$ & $\ldots$ & $\ldots$ \\
\hline GRB 080319B & 0.9382 & $650.6 \pm 33.5$ & $\ldots$ & $\mathrm{C}$ & $\cdots$ & $2.27_{-1.19}^{+1.91}$ & $\cdots$ & $\cdots$ & $\cdots$ \\
\hline XRF 081007 & 0.5295 & $39.9 \pm 9.8$ & 2008hw & $\mathrm{B}$ & $\ldots$ & $1.90 \pm 1.50$ & $\ldots$ & $\ldots$ & $\ldots$ \\
\hline XRR 090618 & 0.54 & $162.0 \pm 3.0$ & $\ldots$ & $\mathrm{C}$ & $\ldots$ & $3.65_{-1.42}^{+2.00}$ & $\ldots$ & $\ldots$ & $\cdots$ \\
\hline XRR 091127 & 0.49044 & $34.0 \pm 1.0$ & $2009 \mathrm{nz}$ & $\mathrm{B}$ & Ic & $1.35 \pm 0.04$ & 8.6 & -0.29 & 0.22 \\
\hline XRF 100316D & 0.0591 & $9.6_{-4.3}^{+9.3}$ & 2010bh & $\mathrm{A}$ & Ic & $1.54 \pm 0.14$ & 8.93 & -0.39 & 0.14 \\
\hline XRR 101219B & 0.55185 & $54.8 \pm 7.8$ & 2010ma & $\mathrm{A} / \mathrm{B}$ & Ic & $1.0 \pm 0.6$ & $\ldots$ & $\ldots$ & $\ldots$ \\
\hline XRR 101225A & 0.847 & $57.0 \pm 24.9$ & $\ldots$ & $\mathrm{D}$ & $\cdots$ & $3.2 \pm 1.6$ & $\cdots$ & $\ldots$ & $\cdots$ \\
\hline GRB 111209A & 0.677 & 768.8 & $2011 \mathrm{kl}$ & $\mathrm{A} / \mathrm{B}$ & $\cdots$ & $\ldots$ & $\cdots$ & -0.39 & $\cdots$ \\
\hline XRR $111228 \mathrm{~A}$ & 0.71627 & $33.8 \pm 4.1$ & $\ldots$ & $\mathrm{E}$ & $\ldots$ & $\ldots$ & $\ldots$ & $\ldots$ & $\ldots$ \\
\hline XRR 120422A & 0.28253 & 97.1 & $2012 \mathrm{bz}$ & A & Ic & $2.55 \pm 0.21$ & 8.95 & -0.4 & 0.4 \\
\hline XRR 120714B & 0.3984 & $71.2_{-8.6}^{+50.9}$ & $2012 \mathrm{eb}$ & $\mathrm{C}$ & Ic & $\ldots$ & $\ldots$ & $\ldots$ & $\ldots$ \\
\hline XRR 120729A & 0.8 & $175.9 \pm 17.6$ & $\ldots$ & $\mathrm{D} / \mathrm{E}$ & $\cdots$ & $\cdots$ & 8.3 & $\cdots$ & 6 \\
\hline XRR 130215A & 0.597 & $101.6 \pm 16.9$ & $2013 \mathrm{ez}$ & $\mathrm{B}$ & Ic & $\ldots$ & $\ldots$ & $\ldots$ & $\ldots$ \\
\hline GRB $130427 \mathrm{~A}$ & 0.3399 & $932.9 \pm 111.9$ & $2013 \mathrm{cq}$ & B & Ic & $6.4 \pm 0.7$ & 9.32 & -0.2 & 0.9 \\
\hline XRR 130831A & 0.4791 & $67.0 \pm 4.0$ & 2013fu & $\mathrm{A} / \mathrm{B}$ & Ic & $\mathbf{1 . 9} \pm 0.9$ & $\ldots$ & $\ldots$ & $\ldots$ \\
\hline XRR 150818A & 0.282 & $99.8 \pm 10.1$ & $\ldots$ & $\mathrm{B}$ & $\cdots$ & $\ldots$ & $\cdots$ & $\cdots$ & $\cdots$ \\
\hline
\end{tabular}

Note. - Grades are classified by Hjorth \& Bloom (2012): (A) Strong spectroscopic evidence; (B) A clear light curve bump as well as some spectroscopic evidence resembling a GRB-SN; (C) A clear bump consistent with other GRB-SNe at the spectroscopic redshift of the GRB; (D) A bump, but the inferred SN properties are not fully consistent with other GRB-SNe or the bump was not well sampled or there is no spectroscopic redshift of the GRB; (E) A bump, either of low significance or inconsistent with other GRB-SNe. Type refers to the supernova explosion type. $E_{K}$ is the supernova ejecta kinetic energy, in the unit of $10^{52}$ erg. $M^{*}$ is the stellar mass of the GRB host galaxy. $Z$ is the metallicity of the GRB host galaxy. SFR is the star formation rate of the GRB host galaxy, in the unit of $M_{\odot} \mathrm{yr}^{-1}$. 
Table 5. Host Galaxy Properties of XRFs and XRRs

\begin{tabular}{|c|c|c|c|c|c|}
\hline Source & $z$ & $E_{\text {peak }}^{o b s}(\mathrm{keV})$ & SFR & $\log \left(M^{*} / M_{\odot}\right)$ & $\log \left(Z / Z_{\odot}\right)$ \\
\hline XRF 050416A & 0.6528 & $14.8_{-7.0}^{+3.6}$ & 2.32 & 9.19 & - \\
\hline XRF 050824 & 0.8278 & 80.0 & - & - & -0.3 \\
\hline XRF 060218 & 0.03342 & $4.7 \pm 0.3$ & 0.05 & 7.78 & -0.53 \\
\hline XRF 061210 & 0.4095 & - & - & 9.52 & - \\
\hline XRF 071031 & 2.692 & $2.1_{-107.5}^{+187.8}$ & - & - & -1.85 \\
\hline XRF 071227 & 0.381 & $35.5 \pm 5.3$ & 0.6 & 10.65 & -0.2 \\
\hline XRF 090205 & 4.6497 & $38.4_{-3.8}^{+5.0}$ & - & 10.83 & - \\
\hline XRF 100316D & 0.0591 & $\begin{array}{l}9.6_{-4.3}^{+9.3} \\
\end{array}$ & 0.14 & 8.93 & -0.39 \\
\hline XRR 050223 & 0.584 & 68.1 & 1.44 & 9.73 & - \\
\hline XRR 050724 & 0.257 & 11.5 & - & 10.64 & - \\
\hline XRR 060614 & 0.125 & $98.5_{-1.8}^{+29.9}$ & 0.01 & 7.95 & - \\
\hline XRR 061006 & 0.4377 & - & 0.17 & 8.86 & - \\
\hline XRR 070306 & 1.4959 & - & 13 & 10.36 & -0.29 \\
\hline XRR 070429B & 0.9023 & $72.9 \pm 13.0$ & - & 10.42 & - \\
\hline XRR 070612 & 0.671 & 137.7 & 81 & - & -0.4 \\
\hline XRR 070724 & 0.4571 & $45.9 \pm 8.6$ & 15.3 & 9.92 & - \\
\hline XRR 070802 & 2.4541 & 58.3 & - & 9.85 & - \\
\hline XRR 080123 & 0.495 & 8.2 & - & 10.02 & - \\
\hline XRR 080325 & 1.78 & - & 9 & 10.8 & - \\
\hline XRR 081109 & 0.979 & $169.7 \pm 42.3$ & 9.9 & 9.82 & - \\
\hline XRR 090417B & 0.345 & 112.9 & - & 10.14 & - \\
\hline XRR 090426 & 2.609 & $55.1 \pm 8.1$ & - & 10.81 & - \\
\hline XRR 091127 & 0.49044 & $34.0 \pm 1.0$ & 0.22 & 8.6 & -0.29 \\
\hline XRR 100621A & 0.542 & $83.0 \pm 9.0$ & - & 8.98 & - \\
\hline XRR 111008A & 4.9907 & $122.6 \pm 29.7$ & - & - & -1.7 \\
\hline XRR $120422 \mathrm{~A}$ & 0.28253 & 97.0 & 0.4 & 8.95 & -0.4 \\
\hline XRR 120729A & 0.8 & $175.9 \pm 17.6$ & 6 & 8.3 & - \\
\hline XRR 130606A & 5.9134 & $150.3 \pm 36.2$ & - & - & -1.1 \\
\hline
\end{tabular}

Note. - SFR is star formation rate of GRB host galaxy, in the unit of $M_{\odot} y r^{-1}$. $M^{*}$ is stellar mass of GRB host galaxy. $Z$ is metallicity of GRB host galaxy. All data are taken from GRB Host Studies (GHostS) database. 